Western University Scholarship@Western

Digitized Theses

Digitized Special Collections

1993

\title{
The Functional Basis Of Counterfactual Thinking
}

Neal Joseph Roese

Follow this and additional works at: https://ir.lib.uwo.ca/digitizedtheses

\section{Recommended Citation}

Roese, Neal Joseph, "The Functional Basis Of Counterfactual Thinking" (1993). Digitized Theses. 2315.

https://ir.lib.uwo.ca/digitizedtheses/2315

This Dissertation is brought to you for free and open access by the Digitized Special Collections at Scholarship@Western. It has been accepted for inclusion in Digitized Theses by an authorized administrator of Scholarship@Western. For more information, please contact tadam@uwo.ca,

wlswadmin@uwo.ca. 


\title{
THE FUNCTIONAL BASIS OF COUNTERFACTUAL THINKING
}

\author{
by \\ Neal J. Roese \\ Department of Psychology
}

Submitted in partial fulfilment of the requirements for the degree of Doctor of Philosophy

Faculty of Graduate Studies The University of Western Ontario

London Ontario

June, 1993

오 Neal J. Roese 1993 
National Library

of Canada

Acquisitions and

Bibliographir Services Branch

395 Wellington Street

Ottawa, Ontario

KIA ONA
Bibliothdque nationale

du Canac.

Direction des acquisitions et

des services bibliographiques

395. ne Wellington

Ottawa (Ontario)

K1A ON4
Your the vorre restrence

Ous his Notreneterence
The author has granted an irrevocable non-exclusive licence allowing the National Library of Canada to reproduce, loan, distribute or sell copies of his/her thesis by any means and in any form or format, making this thesis available to interested persons.
L'auteur a accordé une licence irrévocable et non exclusive permettant d la Bibliotheque nationale du Canada de reproduire, prêter, distribuer ou vendre des coples de sa thèse de quelque manière et sous quelque forme que ce soit pour mettre des exemplaires de cette thèse à la dispocition des personnes intéressées.

L'auteur conserve la propriété du droit d'auteur qui protège sa these. Ni la these ni des extraits substantiels de celle-ci ne doivent être imprimés ou autrement reproduits sans son autorisation.
The author retains ownership of the copyright in his/her thesis. Neither the thesis nor substantial extracts from it may be printed or

otherwise reproduced without his/her permission.

\author{
ISBN $0-315-84003-x$
}




\section{ABSTRACT}

The functional role of counterfactual thoughts ("might have been" reconstructions of the past) was explored in three laboratory experiments. Specifically, counterfactual thoughts were posited to serve two possible functions: an affective function (feeling better) and a preparative function (preparing for the future via avoiding the recurrence of negative events). It is argued that counterfactuals as a generic class of cognitions generally serve these two functions, but that specific types of counterfactuals may in particular do so. Two dimensions are described along which counterfactuals may be classified: direction (upward vs downward) and structure (additive vs subtractive). Upward counterfactuais focus on an alternative that is better than reality, whereas downward counterfactuals focus on an alternative that is worse than reality. Additive counterfactuals focus on the addition of antecedent elements that were not present in the past, whereas subtractive counterfactuals focus on the deletion of antecedent elements that were present in the past.

In all three experiments, these two variables are manipulated, forming, along with self-esteem, $2 \times 2 \times 2$ factorial designs. In Experiment 1, subjects recalled negative life events, generated counterfactuals, and rated their current affect. Direction but not structure influenced affect ratings, such that downward counterfactuals resulted in more positive affect than upward counterfactuals. In Experiment 2, subjects recalled a disappointing examination performance, generated counterfactuals, rated their current affect, and rated their intentions to perform success-facilitating behaviours. Again, direction but not structure influenced affect ratings in the same manner as in Experiment 1. Direction but 
not structure also influenced intention ratings, such that upward counterfactual generation resulted in stronger intentions to perform success-facilitating behaviours. In Experiment 3, subjects engaged in a computer-administered anagram task. Although the affective effects were not significant, both direction and structure influenced performance: upward as well as additive counterfactual generation resulted in greater improvement on the anagram task.

These findings provide initial support for a functional theory of counterfactual thinking: people may strategically use downward counterfactuals to make themselves feel better (an affective function), and they may strategically use upward and additive counterfactuals to improve performance in the future (a preparative function). The present studies suggest that the mechanism underlying the preparative function represents a rausal link from counterfactuals to intentic:as to overt behaviours. Implications for current theory and future research are considered. 


\section{ACKNOWLEDGEMENTS}

First and foremost I would like to acknowledge the incalculable contribution of my advisor, Jim Olson. His powers of insight and articulation have resulted in a collaboration that has been formidable, profitable, and gratifying for the both of us. I have many times felt fortunate to have in Jim not only an advisor but also a role model of compleat academic, exceptional father, and allaround great guy. Had I not teamed up with Jim in 1990, my graduate school experience would doubtless have been a dim one.

I would also like to thank my committee members -- Mike Atkinson, Vicki Esses, Ed Grabb, Albert Katz, Dale Miller, and Clive Seligman -. for their efficient and effective handling of what may otherwise have been a tortuous process.

The final months of my seemingly endless years of education have been occasionally challenging but primarily recreational; the credit for this may be attributed largely to the talents of my art, wine, and music advisor, Vuk Vuksanović, and my videogame and fish consultant, Steve Hanna. I am also grateful to the wisdom, weirdness, and intellectual camaraderie of two long distance compatriots, Ron Deibert (the piper at the gates of global satellite surveillance) and Steve Wiseman (the last Freudian).

The completion of this dissertation was eased considerably by cerrain intangible elements that have contributed importantly to the spirit of this work (or have at least provided sufficient refuge from its drudgery that $I$ could return refreshed to it the next day). Such intangibles include the 4th movement of Mahler's fifth, the entirety of Bruckner's ninth, the children of Rushdie's midnight, 
the good perple of the Burgundy region, the fettucini fiorentina at Piccolos, Mark's stuff, big budget American war movies, the sculptures of Pericletian Athens, and a videogame called Rampart.

I owe my greatest debt to my parents, Joseph and Geraldine, whose keen combination of exceptional genes and stimulating childhood encouragement precipitated the events leading ultimately to this dissertation. Without them, of course, none of this would be possible.

Most of all, I thank Karen, my fiancee, closest friend, and life partner. Attaining this uream was made infinitely easier through her kindness, integrity, and close support. This dissertation, my research, my career, and my achievements, past, present, and future, are dedicated to her and to our children. 
TABLE OF CONTENTS vi

LIST OF TABLES viii

LIST OF FIGURES x

LIST OF APPENDICES $\mathbf{x i}$

CHAPTER I - INTRODUCTION

Counterfactual Thinking

The Direction of Counterfactual Thoughts

The Structure of Counterfactual Thoughts

Mechanism of the Preparative Function

Prefactual Thinking

Overview and General Hypotheses

CHAPTER II - EXPERIMENT 1

Method 28

Results 33

Discussion

CHAPTER III - EXPERIMENT 2

Method

Results

Discussion 


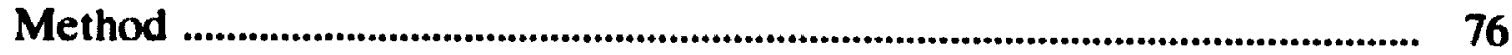

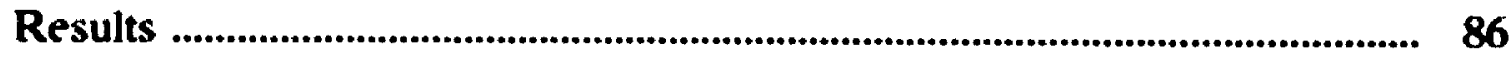

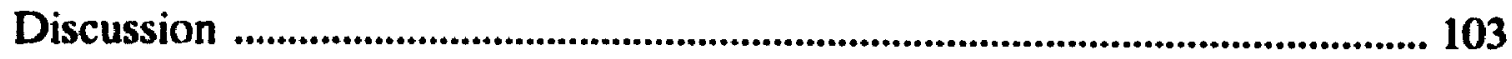

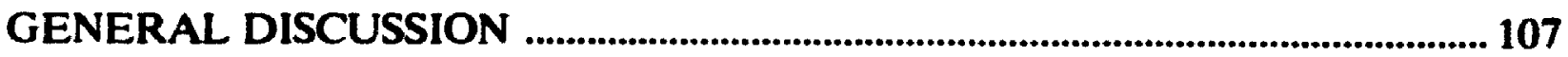

The Affective Function ........................................................................................108

The Preparative Function ...............................................................................111

The Interplay Between Affective and Preparative Processes .................................................................. 116

Conceptual Limitations of the

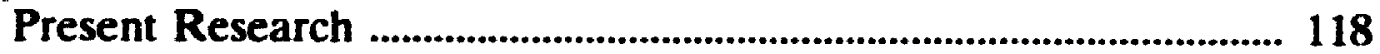

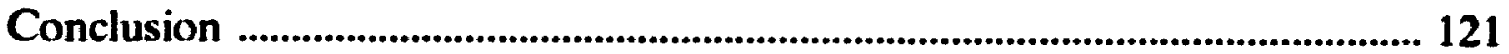

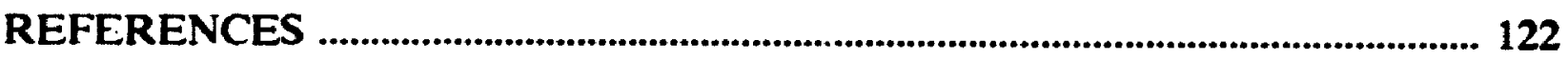

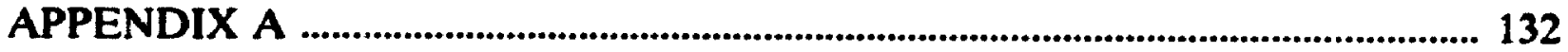

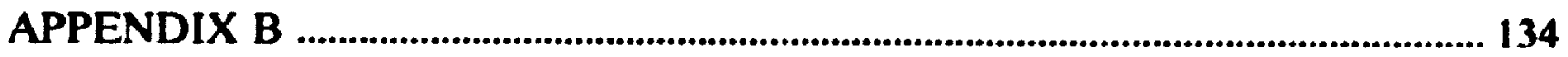

APPENDIX C

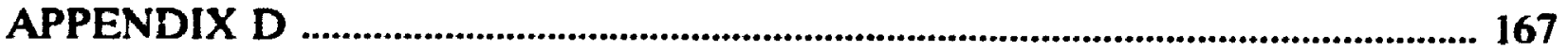

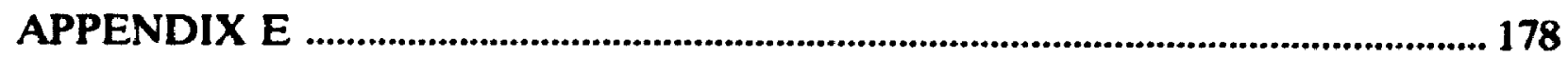

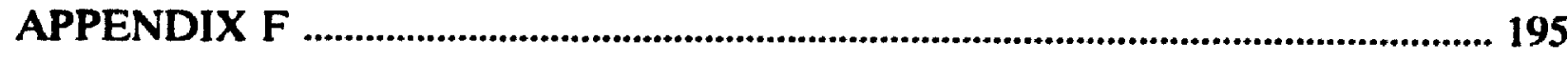

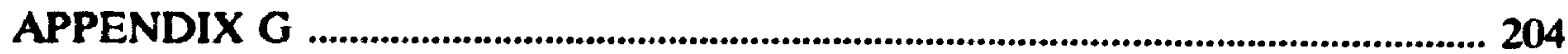

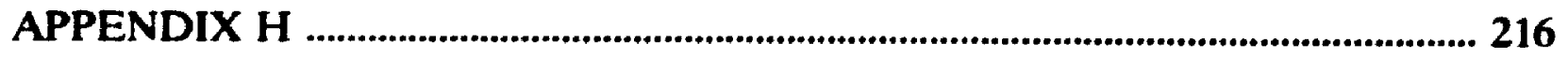

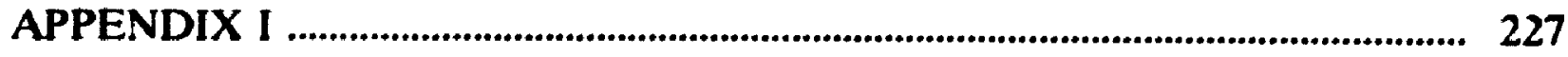

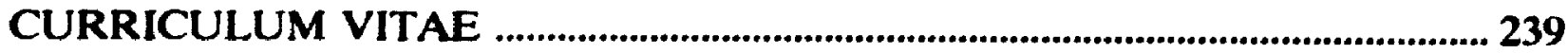




\section{LIST OF TABLES}

Table

Description

Page

Experiment 1

1 Summary of within-cell means for the affective measures

2 Correlations between counterfactual direction, structure, affect, and attributional ratings

\section{Experiment 2}

3 Summary of within-cell means from the principal dependent measures

4 Correlations between counterfactual direction, structure, affect, and intention ratings

5 Correlations between counterfactual direction, structure, affect, and attributional ratings

\section{Experiment 3}

6 Anagrams (with solutions) used in Experiment 3

7 Interaction effect between direction, structure, and

$\mathrm{SE}$ on affect ratings

8 Summary of within-cell means from the affect ratings

9 Summary of within-cell means from the performance measures

10 Interaction effect between direction, structure, and

SE on performance

11 Correlations between counterfactual direction, structure, affect, expectancy ratings, and performance

12 Correlations between counterfactual direction, structure, affect, and attributional ratings 


\section{LIST OF FIGURES}

Figure

Description

Page

1 Hypothesized mechanism linking counterfactuals to behaviour

19

2 Effect of counterfactual direction on global affect scors

3 Effect of counterfactual direction on commissional intention ratings 60

4 Effect of counterfactual direction on performance 93

5 Effect of counterfactual structure on performance 94 


\section{LIST OF APPENDICES}

Appendix

Description

Page

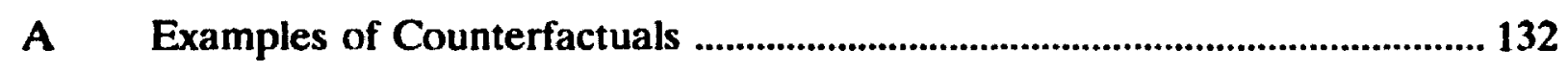

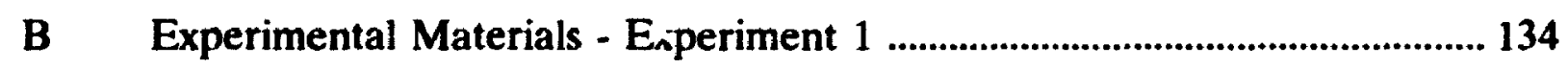

C Experimental Materials - Experiment 2 ...................................................... 148

D Experimental Materials - Experiment 3 ............................................ 167

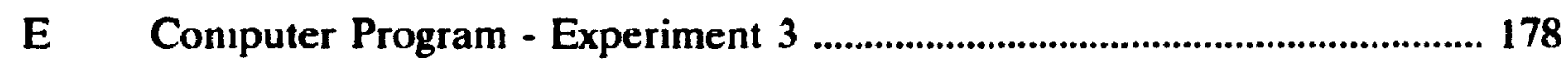

F Analysis of Variance Summary Tables - Experiment 1 ............................ 195

G Analysis of Variance Summary Tables - Experiment 2 ........................... 204

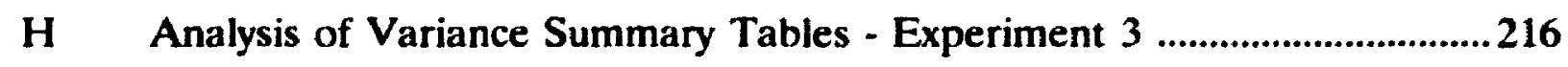

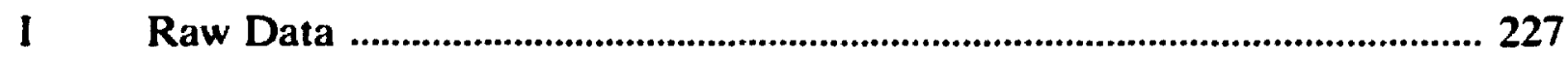


The author of this thesis has granted The University of Western Ontario a non-exclusive license to reproduce and distribute copies of this thesis to users of Western Libraries. Copyright remains with the author.

Electronic theses and dissertations available in The University of Western Ontario's institutional repository (Scholarship@Western) are solely for the purpose of private study and research. They may not be copied or reproduced, except as permitted by copyright laws, without written authority of the copyright owner. Any commercial use or publication is strictly prohibited.

The original copyright license attesting to these terms and signed by the author of this thesis may be found in the original print version of the thesis, held by Western Libraries.

The thesis approval page signed by the examining committee may also be found in the original print version of the thesis held in Western Libraries.

Please contact Western Libraries for further information:

E-mail: libadmin@uwo.ca

Telephone: (519) 661-2111 Ext. 84796

Web site: http://www.lib.uwo.ca/ 


\section{CHAPTER I}

INTRODUCTION 
If matters had fallen out differently, she wondered, might she not have met some other man? She tried to picture to herself the things that might have been -- that different life, that unknown husband. He might have been handsome, intelligent, distinguished, attractive ...

-- Flaubert (1857/1950), p. 57.

Tales of human suffering are replete with examples of thoughts of what might have been, of what could have occurred if only a few subtle details had been different. The tormented mistress of Flaubert's novel, Madame Bovary, bored with her provincial life, dreams of a better one and a better husband. The ability to imagine alternative, or counterfactual, versions of actual events appears to be a pervasive, perhaps even essential, component of human consciousness (e.g., Hofstadter, 1979). Social psychologists are increasingly interested in this propensity to react to various life events with thoughts of "what if" or "if only" (e.g., Kahneman \& Miller, 1986; Miller, Turnbull, \& McFarland, 1990). Once in mind, these counterfactual thoughts influence a range of judgments and affective reactions, including causal ascriptions (Kahneman \& Varey, 1990; Lipe, 1991; Mackie, 1974; Miller, Taylor \& Buck, 1991; Wells \& Gavanski, 1989), selfperception (Roese \& Olson, 1993b), sympathy and victim compensation (Miller \& McFarland, 1986), suspicion (Miller, Turnbull, \& McFarland, 1989; but see Kirkpatrick \& Epstein, 1992; Pipes et al., 1991) regret (Landman, 1987), and happiness (Johnson, 1986). 
But what essential purposes are served by the ability to reason counterfactually? Are such thoughts globally beneficial, do they hamper goaldirected behaviour, or are they merely epiphenomenal and hence inconsequential? A decade of research has illuminated a variety of situational constraints on and affective consequences of counterfactual thinking, but little attention has been directed toward the functional basis of such thoughts. The goal of the present program of research is to examine two possible functions that counterfactual thoughts may serve: an affective function (feeling better) and a preparative function (avoiding the recurrence of negative outcomes). Although counterfactuals as a generic class of cognitions may generally serve these two functions, the present research aims to provide a more exact theoretical specification of the operation of these functions. The approach taken is to classify counterfactuals into specific subtypes, then link these subtypes to one or the other function. Two dimensions form the basis for this classification: counterfactual direction (namely, counterfactuals that focus on improving versus worsening reality) and counterfactual structure (namely, counterfactuals that focus on adding versus subtracting antecedent events).

For present purposes, the term function is used to denote any cognitive process that is useful or beneficial on a global level. Thus, as with previous functional approaches, counterfactual processes are examined and explained in terms of the "needs" they serve. As Katz (1960) put it, "the functional approach is the attempt to understand the reasons people [behave] as they do. The reasons, however, are at the level of psychological motivations and not the accidents of external events and circumstances" (p. 170). Functionalism as a psychological school of thought, advanced in the late 19th century by John Dewey, William 
James, and others, focused on what each of many specific mental acts and behaviours "is for" (Boring, 1957, p. 555). Much of this work was informed by Darwinian evolutionary theory. Hence, behaviours were characterized in terms of their adaptive signits:ance (e.g., fight or flight reactions that are crucial to the survival of individual organisms). However, the evolutionary implications of counterfactual functionality are beyond the scope of the present research, and hence are not considered in detail.

In the pages that follow, I will first review previous experimental explorations of counterfactual thinking. The dimension of counterfactual direction is then examined and used to make predictions regarding the affective and preparatory functions. Next, the dimension of counterfactual structure will be considered in detail, and further functional predictions are derived on the basis of this variable. Last, the literature examining prefactual thinking is reviewed and compared to the counterfactual literature. This literature forms the basis for predictions regarding the mechanism by which counterfactuals may serve a preparative function.

\section{Counterfactual Thinking}

Counterfactuals are examples of logical propositions called conditionals (Kvart, 1986; Lewis, 1973; Reisenbach, 1976). As such, they contain both an antecedent (e.g., "If only I had studied") and a consequent (e.g., "then I would have passed"). A central concept within this literature has been that of mutability; that is, the ease with which an antecedent may be cognitively altered to construct the counterfactual statement (Kahneman \& Miller, 1986). For the average person, some things appear to be very mutable (e.g., effort), whereas other things 
seem relatively immutable (e.g., the colour of the sky). Of course, with some effort it is possible to mutate imaginatively virtually any event or characteristic, but the utility of the mutability construct derives from the observation that there are regularities to the frequency and content of counterfactuals generated in specific situations, and that, as Hofstadter (1985) argued, there appear to be natural "fault lines of the mind" along which reality is cognitively dissected (p. 239). Charting these fault lines hy identifying predictors of mutability has been the dominant focus of counterfactual research by social psychologists.

Several variables influence mutability. Recent research indicates that people find exceptional antecedents to be more mutable than routine antecedents (Gavanski \& Wells, 1989; Kahneman \& Tversky, 1982b; Wells, Taylor \& Turtle, 1987), and overt acts to be more mutable than failures to act (Gleicher, Kost, Baker, Strathman, Richman, \& Sherman, 1990; Kahneman \& Miller, 1986; Landman, 1987). People are more likely to create counterfactuals by undoing the initial rather than the final event in a causal chain (Wells et al., 1987), but to undo the final rather than the initial event in a noncausally linked chain of events (Miller \& Gunasegaram, 1990). Counterfactual generation may also be greater following negative than positive outcomes (cf. Bouts, Spears, \& van der Pligt, 1992; Epstein, Lipson, Holstein, \& Huh, 1992; Gleicher et al., 1990; but see Markman, Gavanski, Sherman, \& McMullen, 1993; Roese \& Olson, 1993b, 1993c, for disconfirming evidence).

Other research indicates that the content and quantity of counterfactuals mediate the emotional impact of a given outcome. Kahneman and Miller (1986) posited a principle of emotional amplification, asserting that greater mutability of a. Itecedent events leads to greater counterfactual availability, which in turn 
increases perceived affective intensity. In other words, outcomes that are more easily undone elicit stronger emotional reactions. In several studies, people perceived others to be more upset by an accident preceded by exceptional rather than typical antecedents (Kahneman \& Miller, 1986). Manipulations of the act versus non-act variable produced similar emotional effects: Scenarios that described an individual who performed an act leading to a negative outcome elicited greater perceived regret than scenarios in which a negative outcome occurred after an individual considered performing an act but then decided against performing it (Gleicher st al., 1990; Kahneman and Tversky, 1982a; Macrae, 1992; Macrae \& Milne, 1992; Landman, 1987; see also Girotto, Legrenzi, \& Rizzo, 1991).

The potentially important role of the self in counterfactual generation has received less attention, even though the very nature of "if only" thoughts may frequently carry crucial implications for individuals' self-concepts. Regardless of the exceptionality or overtness of antecedent actions, the cognition that, for example, "I could have done something to prevent this," may have markedly different consequences to the extent that the outcome in question is relevant to the self (Bulman \& Wortman, 1977). Consistent with this idea, self-esteem has been found to moderate the content of counterfactuals generated following positive versus negative self-relevant outcomes. In studies employing a scenario methodology, people with high self-esteem (HSE) were more likely than people with low self-esteem (LSE) to mutate their own actions following success (thereby taking credit for the success). LSE persons, however, tended to internalize failure, in that they were more likely than high self-esteem persons to mutate their own actions following failure (Roese \& Olson, 1991, 1993b). Although these findings 
may be interpreted in self-presentational or information processing terms, the motivational interpretation is particularly relevant to the present discussion. HSE people likely possess greater motivation to protect and enhance positive evaluations of themselves than LSE penple, resulting in positively biased illusions of themselves and their futures (Taylor \& Brown, 1988). Thus, counterfactual generation by HSE persons may directly serve the function of maintaining positive affect and positive self-views. More generally, this research represents an initial step toward a functional theory of counterfactual thinking, showing personality differences in affective regulation.

Overall, current research suggests that counterfactual thinking is a common response to surprising or negative outcomes, and that it can carry important affective consequences for the individual. On a more general level, counterfactual thinking is closely tied to our knowledge of the world and our future lives within that world. Linguist George Steiner wrote:

It is unlikely that man, as we know him, would have survived without the fictive, counter-factual, anti-determinist means of language, without the semantic capacity, generated and stored in "superfluous" zones of the cortex, ... to articulate possibilities beyond the treadmill of organic decay and death $(1975$, p. 227).

Steiner alludes to the preparative function of counterfactual thinking, one that allows people to consider past mistakes as well as past triumphs, synthesizing them into prescriptions for the future that may facilitate success in that future.

The functionality of counterfactual thinking is reminiscent of the survival value posited for attributional thinking. Attributions represent the search for "why"; that is, the identification of the causes of specific outcomes. Understanding 
why something occurred is clearly beneficial: "The attributor is not simply ... a seeker after knowledge; his latent goal in attaining knowledge is that of effective management of himself and his environment" (Kelley, 1971, p. 22). Without such predictive management skill, people "could neither avoid nor prevent, but would be at the mercy of seemingly fickle events in the environment" (Heider, 1958, p. 257). Attribution theorists, from the earliest writings of Heider through to the contemporary models of Weiner (1985), have pointed to the inherent functional value of causal reasoning via its capacity to engender prediction and control. Such causal reasoning is directly connected to counterfactual thinking. Recent research by Wells and Gavanski (1989) showed that counterfactual reasoning is an important mechanism by which antecedents are inferred to cause outcomes (see also Lipe, 1991; Mackie, 1974; McGill \& Klein, 1993). To be more specific, the judgment that outcome $\mathrm{Y}$ was caused by antecedent $\mathrm{X}$ may follow from the counterfactual inference that removing $X$ undoes $Y$ (i.e., if $X$ had not been present, $Y$ would not have occurred). Thus, it may be the mechanism of counterfactual judgment that forms the basis for the functional value of attributions previously posited by Heider, Kelley, Weiner, and other theorists.

The Direction of Counterfactual Thoughts

The assertion that counterfactual thinking in general may serve to enhance performance may be tenable, but the goal of the present research is to specify subtypes of counterfactuals that may be especially likely to serve this goal. Toward this end, the preparative function may best be served by "upward" counterfactuals, those that describe alternatives that are better than what actually happened, as opposed to "downward" counterfactuals, which describe alternatives 
that are worse than what actually happened. Upward alternatives may be taken as schemata for future action, making salient those scripts that are necessary to facilitate success. Consequently, "the realization of positive alternatives should make positive outcomes more likely in the future" (Johnson \& Sherman, 1990, p. 512; see also Folger, 1984). Indeed, upward counterfactuals may underlie many of the more imaginative, passionate, and ambitious of human pursuits. They "make up a grammar of constant renewal. They force us to proceed afresh in the morning, to leave failed history behind. Otherwise our posture would be static and we would choke on disappointed dreams" (Steiner, 1975, p. 217).

Markman et al. (1993) have provided the most convincing demonstration of this reasoning to date. In their laboratory experiment, subjects played a computerized blackjack game for real money, receiving outcome feedback that, although objectively neutral, was framed either as a win, as a loss, or as neutral. Subjects were more likely to spontaneously generate upward counterfactuals following failure than in either of the two other conditions. More interesting was the observation that subjects who expected to play the game again generated more upward counterfactuals than those not expecting to play again. The inference of a preparatory function was indirect: subjects with no expectation of playing again had no "need" to prepare for the future, hence the exploration of upward possibilities was of lesser utility. Subjects expecting to play again, in contrast, generated upward counterfactuals, perhaps in order to maximize their chances of winning more money in the next blackjack game. Although plausible and consistent with the theoretical position expounded here, a more direct demonstration of the causal impact of upward versus downward counterfactuals on objective performance is needed. Such is one purpose of the present program of 
:esearch.

That upward counterfactuals serve a preparative function also finds indirect support in the social comparison literature. Researchers examining coping with negative life events have interpreted upward social comparison -- that is, comparison with soneone who is better off than oneself -- as providing the most useful information "for potential survival and successful coping" (Taylor \& Lobel, 1989, p. 573). When things are likely to improve, people are even more likely to draw preparative information from upward comparisons (Buunk, Collins, VanYperen, Taylor, \& Dakof, 1990; Hemphill \& Lehman, 1991; Taylor, Buunk, \& Aspinwall, 1990). Indeed, the functional nature of upward comparisons was implicit in Festinger's (1954) hypothesis of a unidirectional drive upward, in which people are motivated to become more capable than current performance levels, and hence are most likely to look toward more capable others for comparisons. The information gleaned from such comparisons can then be employed for selfbetteıment. Self-focusing counterfactuals are similar to social comparisons, differing only in that the comparison is made to an alternative self rather than to another individual. It seems likely, then, that both upward counterfactuals and upward social comparisons may serve the same preparative function.

Counterfactual thinking may also serve an affective function. That is, people may imagine how things could have been different in order to make themselves or others feel better. The dimension of counterfactual direction is very likely linked to this affective function as well (Johnson \& Sherman, 1990). By way of a simple contrast effect, downward counterfactuals (comparisons between a given outcome and a worse alternative) should tend to elicit positive affect (e.g., relief), whereas upward counterfactuals (comparisons between an outcome and a 
better alternative) should tend to elicit negative affect (e.g., disappointment, regret).

Several previous experimental findings are consistent with this logic. Rape victims appear to draw comfort by noting that they could have been more seriously injured or even killed (Burgess \& Holmstrom, 1979). Cancer patients often reason that their illness could be worse or that they could already have died, resulting in more posıtive feelings (Taylor, Wood, \& Lichtman, 1985). Emotional regulation of this sort was hypothesized by Taylor and Schneider (1989) to underlie both counterfactual generation and social comparisons, in that a downward focus may be affectively self-enhancing and thus particularly likely under conditions of threat (Wills, 1981).

The findings of Markman et al. (1993) supported this contention also. These researchers found that the manipulations that influenced counterfactual direction (i.e., outcome frame and expectancy of repeating the task) also influenced expressions of satisfaction. Thus, negative outcomes and expectations of repetition not only evoked more upward counterfactuals, but greater feelings of dissatisfaction as well. Partialling out the relative contributions of outcome frame and expectancy still yielded a significant negative correlation between direction and satisfaction. These findings support the hypothesis that counterfactual direction causally influences affect. One goal of the present research is to document this relation more clearly using a more general measure of affect and a more direct manipulation of counterfactual direction.

Although the link between direction and affect has been frequently demonstrated by social comparison researchers (e.g., Crocker, Thompson, McGraw, \& Ingerman, 1987; Wood, Taylor \& Lichtman, 1985), more recent 
theorists have noted that the affective consequences of social comparison are not inherently linked to direction (Nosanchuk \& Erickson, 1985; Taylor, Buunk, \& Aspinwall, 1990). Making an upward social comparison not only yields information conducive to negative affect (e.g., that others are better off than oneself), but also information that may have positive affective consequences (e.g., that it is possible for things to improve in the future). That is, the cognition that "things could have turned out better" may be upsetting because it makes salient the deprived present state, yet it may also be uplifting to the extent that it leads to the expectation of future betterment. Interestingly, Buunk et al. (1990) found that self-esteem moderated the affective impact of social comparisons, such that HSE individuals were more likely to draw positive affect from both upward and downward comparisons than LSE individuals (see also Crocker et al., 1987). This was particularly true for upward comparisons -- HSE persons were much !ess likely to experience the pain of gazing upon more fortunate others because they were more likely to focus on the positive implication that they themselves may be similarly fortunate in the future. In this way, HSE persons appear to be less vulnerable to any negative affective consequences of social comparison. It therefore seems possible that self-esteem may predict not only the content of counterfactual thought following various outcomes (e.g., Roese \& Olson, 1993b), but also the affective consequences of upward and downward counterfactuals, such that HSE persons may report more positive affect than LSE persons following either upward or downward thoughts.

\section{The Structure of Counterfactual Thoughts}

Counterfactual structure, as defined here, is rooted in several related 
concepts expounded in earlier writings. Kahneman and Tversky (1982b) proposed that the alterations people make in constructing counterfactuals can be classified into three types. "Uphill" changes are the addition of unlikely antecedent events; "downhill" changes are the deletion of unlikely antecedent events; and "horizontal" changes are alterations to a continuous variable, such as changes in time or quantity of an occurrence. For example, if a student fails a test, a corresponding uphill counterfactual might be "If only I had purchased extra study materials"; a downhill change might be "If only I didn't have to help my sick roommate"; and a horizontal change might be "I should have begun studying at 6 PM rather than 10 PM". Kahneman and Tversky proposed that downhill changes are more common than uphill or horizontal changes (see also Wells et al., 1987), an assertion based largely on the salience and mutability of exceptional as opposed to normal antecedents. The logic of the uphill versus downhili distinction was obscured somewhat by Kahneman and Tversky with their confounding of exceptionality / normality with addition / subtraction. What these authors meant was that the addition of an exceptional antecedent is less likely than the addition of a normal antecedent (i.e., uphill changes are less frequent), and that the subtraction of an exceptional antecedent is more likely than the subtraction of a normal antecedent (i.e., downhill changes are more frequent). This assertion is supported by the literature, but when rephrased in this way, it becomes clear that exceptionality/normality and not addition/subtraction is the basis for the predicted effect (cf. Dunning \& Parpal, 1989).

Unfortunately, the confusion with respect to the separate roles of these two dimensions resulted in the more questionable assertion that subtractions are more common than additions, repackaged by Kahneman and Miller (1986) in terms of 
the hypothesis that counterfactual thoughts are more easily generated for outcomes that follow from an overt action (i.e., an act of commission) than from a failure to act (i.e., an act of omission), because mutations of actions involve subtractions whereas mutations of inactions involve additions. Attempts to demonstrate this extension have been indirect. Kahneman and Miller also advanced the principle of emotional amplification, which posits that more mutable outcomes (or heightened counterfactual generation) result in perceptions of greater affective intensity. Accordingly, several studies have shown that failure preceded by acts of commission (which should heighten counterfactual generation) produced greater regret than failure preceded by acts of omission (e.g., Gleicher et al., 1990; Kahneman and Tversky, 1982a; Landman, 1987).

Kahneman and Tversky's (1982b) original theoretical formulation of uphill, downhill, and horizontal changes was predicated on their assumption of a general tendency to construct counterfactuals by changing exceptional or unexpected antecedents into more normal or expected antecedents. Gavanski and Wells (1989), however, noted that these principles may be restricted to cases where the outcome is also exceptional (e.g., a fatal automobile accident). They replicated Kahneman and Tversky's (1982b) findings for exceptional outcomes, but found that people mutated prior events in the direction of exceptionality (i.e., by adding exceptional or deleting normal antecedents) when mentally undoing normal outcomes. Gavanski and Wells concluded that counterfactual processing may be guided in part by an assumption that exceptional outcomes follow from exceptional antecedents, whereas normal outcomes follow from normal antecedents.

Extending Gavanski and Wells' (1989) logic, Roese and Olson (1993c) 
suggested that a simple and conceptually clear distinction could be made based only on the addition versus subtraction dimension, independent of the normality versus exceptionality dimension. Specifically, counterfactual thoughts can take either an additive, subtractive, or substitutional form. Additive counterfactual structures are those that add elements in order to reconstruct reality (e.g., "If only I owned an umbrella, I would not have gotten wet"), whereas subtractive structures are those that remove elements to reconstruct reality (e.g., "If only it hadn't rained today, I wouldn't have gotten wet"). When both are combined so that an addition replaces the subtraction, a substitutional structure results (e.g., "If only I had driven to work instead of bicycling, I would not have gotten wet"). Focusing only on the additive versus subtractive distinction, several novel predictions emerge.

Perceptions of success and failure diverge (e.g., Miller \& Ross, 1975). Not only do people tend to attribute their own success internally and their own failure externally (i.e.. a self-serving bias), but they also view the antecedents to success and failure differently. For most, success is intended and expected (Parducci, 1968); hence, success may typically be seen as the result of deliberate actions. Failure, however, is unwanted and unexpected; people may therefore tend to see it as resulting from the failure to act appropriately. Further, people in our culture generally value internally-driven actions (Jellison \& Green, 1981) and seem to subscribe to a principle of "control," whereby positive action is good (e.g., "Don't just stand there -. do something"). Also, people often overestimate the degree to which outcomes can be controlled (Langer, 1975). These various factors may generate the perception that success and failure result from the presence or absence, respectively, of appropriate, planned acts. 
This reasoning suggests a divergence in the counterfactual structures people will use to undo success and failure. Specifically, undoing success should typically involve removing a successful antecedent action (i.e., a subtractive counterfactual, iuch as, "If I hadn't studied, I would have failed"). Undoing failure, on the other he nd, should typically involve adding a new antecedent action that would have enhanced the chances of success (i.e., an additive counterfactual, such as, "If I hau bought the study guide, I would have passed"). Several studies indeed demonstrated that additive counterfactuals were more frequent after negative outcomes, whereas subtractive counterfactuals were more frequent after positive outcomes (Roese \& Olson, 1991, 1992, 1993b, 1993c). Substitutions were found to be extremely rare in any case. Also, failures framed by a history of past failure generated an even greater number of additive counterfactuals (Roese \& Olson, 1993c). This pattern was interpreted in terms of a preparative function of counterfactuals, served in particular by the additive structure. Specifically, if additive structures identify specific means of avoiding the recurrence of unpleasant past experiences, then when failure becomes repeated or habitual, people may be especially likely to identify ways of avoiding future failure.

There are two reasons why additive counterfactuals might better serve a preparative function than subtractive counterfactuals. First, as implicitly noted, additive counterfactuals are more specific. They focus on one response option that might have resulted in success, and hence should be implemented in the future, whereas subtractive counterfactuals merely remove one previous response option from consideration. Second, and perhaps more important, additive counterfactuals are more creative. Whereas subtractive counterfactuals are restricted to the original set of premises (i.e., what really happened), additive 
counterfactuals are, by definition, those that go beyond the original premise set, fabricating novel options perhaps never considered in the past. According to this reasoning, and consistert with the findings of Roese and Olson (1993c), additive counterfactuals may represent a more effortful, systematic mode of processing than subtractive counterfactuals.

\section{Mechanism of the Preparative Function}

Both the dimensions of counterfactual direction and structure have been posited to play a role in the preparative function. Specifically, both upward and additive counterfactuals were predicted to engender improvement in future performance. But how might such effects occur? The most likely mechanism by which upward and additive counterfactual generation might enhance performance is via their generation of plans or scripts for the future (Johnson \& Sherman, 1990). These counterfactuals are perhaps easily converted into prefactual thoughts (conditional statements focusing on future possibilities), which are causally linked to intentions to perform success-facilitating behaviours, which are in turn linked to overt behaviour, and finally to enhanced performance. This pathway is illustrated in Figure 1. The causal linkage between prefactuals and behavioral intentions and overt behaviour has been demonstrated frequently in a body of literature reviewed below.

\section{Prefactual Thinking}

Conditional thinking (of which counterfactuals are one type) is not restricted to cognitive manipulations of past occurrences. Indeed, much conditional thinking focuses on future possibilities, on "what if" types of scenarios. 
Figure 1

Hypothesized mechanism linking counterfactuals to behaviour

COUNTERFACTUAL

"If Id done X..."

PREFACTUAL

"If I do X..."

INTENTION

"Tm going to do $X . "$

BEHAVIOUR

Doing $\mathbf{X}$ 
For example, imagining consequences of buying a winning lottery ticket two weeks from now is structurally similar to imagining having bought a winning ticket two weeks ago. I term such conditional statements, which focus on future rather than past occurrences, "prefactuals," thus differentiating them within the broader class of expectancies and imaginings. These types of thoughts have not been explicitly connected to research on counterfactuals, although some authors (Johnson \& Sherman, 1990; Kahneman \& Tversky, 1982b; Taylor \& Schneider, 1989) consider both to fall under the general umbrella category of mental simulations. As will be argued shortly (and consistent with the discussion of Johnson \& Sherman, 1990), considerations of the functional basis of both types of conditional thoughts provide a conceptual connection between counterfactuals and prefactuals that extends beyond their similarity in logical structure.

People imagining the occurrence of a future personal event come to see that event as more likely to occur than those not imagining it (e.g., Sherman, Skov, Hervitz, \& Stock, 1981; Sherman, Zehner, Johnson, \& Hirt, 1983). For example, subjects who imagined their involvement in an automobile accident were later more likely to expect a future automobile accident than control subjects (Gregory, Burroughs, \& Ainslie, 1985). Carroll (1978) interpreted this finding in terms of the availability heuristic (Tversky \& Kahneman, 1973), such that imagining a possibility makes the details of that possibility more available in memory, which in turn enhances its perceived likelihood. These effects tend to occur only when people imagine themselves as the main character in hypothetical scenarios (Anderson, 1983), and when the scenarios are commonplace and hence more easily imaginable (Sherman, Cialdini, Schwartzman, \& Reynolds, 1985). More important, several studies have shown that imagining hypothetical 
scenarios may also increase the probability that corresponding behaviours will occur (Campbell \& Fairey, 1985; Sherman et al., 1981). In a field study by Gregory, Cialdini, and Carpenter (1982), homeowners who were contacted regarding cable television service were more likely to subscribe to such a service if they had initially been asked to imagine themselves enjoying the benefits of cable television. Sherman and Anderson (1987) found that patients who imagined themselves showing up for regular psychotherapy sessions exhibited about half the rate of dropout as control subjects.

A study by Sherman et al. (1981) is a particularly clear example of the experimental logic underlying behavioural demonstrations of prefactual effects. Before engaging in an anagram-solving task, subjects imagined performing either very well or very poorly on the task and wrote hypothetical explanations for this outcome. Control subjects neither imagined nor explained any outcome. Those who imagined and explained success not only expected superior performance, but also performed better than control subjects. Those who imagined and explained failure performed worse than control subjects. Prefactuals may thus link cognition to behaviour by creating a plan that is both efficient and constrained by expected feasibility (e.g., Folger, 1984; Taylor \& Schneider, 1989).

Like counterfactuals, prefactuals are examples of conditional statements. For example, the crux of the Gregory et al. (1982) study is subjects' perceptions that "If I get cable TV, then I will enjoy many programming benefits". Of course, people may not necessarily frame their thoughts in exactly this way, perhaps leaving the antecedent or the consequent to be merely implicit, but the basic conditional framework remains the same.

The causal pathway iinking prefactual thinking, perceived likelihood (or 
behavioural intention), and overt bejaviour points to the preparative function of these prefactual thoughts, at least to the extent that they focus on a positive or desired future possibility. If prefactuals illuminate actions that may in the future avoid negative outcomes (e.g., illness or hardship) or facilitate positive future outcomes (e.g., academic success), the perceived likelihood as well as the actual occurrence of these desired positive outcomes are increased. It has already been argued that counterfactuals may serve this same function, that is, to identify ways that life may improved in the future. Thus, it may appropriately be posited that the conversion of counterfactuals (i.e., what might have been) into prefactuals (i.e., what could be) constitutes the mechanism by which counterfactuals influence (and may indeed enhance) performance (see Figure 1).

To assess this mechanism, the general strategy of the present experiments was to manipulate the consideration of those counterfactual subtypes posited to play a role in the preparative function (i.e., upward as opposed to downward counterfactuals; additive as opposed to subtractive counterfactuals). First, counterfactuals of these subtypes were predicted to have a causal influence on intentions of performing behaviours that are facilitative of success. That is, upward and additive counterfactuals were expected to result in heightened behavioural intentions to perform facilitative actions. Second, counterfactuals of these subtypes were predicted to have a causal influence on overt performance. That is, upward and additive counterfactuals were expected to result in enhanced performance on a skills-based task.

\section{Overview and General Hypotheses}

Three experiments were conducted that explored the two possible 
functional bases, affective and preparative, of counterfactual thinking. In all three experiments, the approach taken was to manipulate counterfactual direction and structure. That is, on a between-subjects basis, subjects were requested to record counterfactuals that were either upward or downward, and orthogonal to this factor, either counterfactuals that were either additive or subtractive. In addition, the individual difference variable of self-esteem was assessed for its possible moderating role in the predicted functional effects. Since self-report SE scores were used to divide people, using a median split, into high and low self-esteem groups, all three experiments constituted 2 (direction) $\times 2$ (structure) $\times 2$ (SE) fully factorial designs.

In Experiment 1, subjects recalled and briefly described a recent, negative life event. They were then requested to generate and record counterfactual alternatives to the event recalled. Each subject was requested to consider only one of the four possible subtypes of counterfactuals from the 2 (direction) $\times 2$ (structure) matrix. Subjects then rated their current affect. In Experiment 2, subjects recalled a recent examination on which they performed poorly. The counterfactual alternatives to the performance that they imagined were again manipulated; subjects then completed an affect measure and made likelihood estimates of performing several behaviours facilitative of future success on exams. In Experiment Three, subjects worked on a computer-administered anagram task, which was followed by failure feedback. Subjects then generated counterfactual alternatives to their performance, rated their current affect, then completed a second set of anagrams. Improvement in performance from the first to the second set of anagrams constituted the principal dependent measure in this third experiment. 
Three main hypotheses guided these experiments. First, consideration of upward counterfactual thoughts was predicted to cause more negative affect than consideration of downward counterfactual thoughts (all three experiments). The factor of counterfactual structure was not predicted to play a role in the affective function. Second, upward counterfactuals, relative to downward counterfactuals, were predicted to result in heightened intentions to perform success-facilitating behaviours (Experiment 2 ) and in greater improvements in performance (Experiment 3). Third, additive counterfactuals, relative to subtractive counterfactuals, were also predicted to result in heightened intentions to perform facilitative behaviours (Experiment 2) and in greater improvements in performance (Experiment 3). No interactions between these two manipulated variables were anticipated.

A secondary, more exploratory focus of this research was the possible moderating role of self-esteem in the affective consequences of considering these different subtypes of counterfactual thoughts. HSE persons may be more likely than LSE persons to report positive affect following the consideration of any negative outcome (i.e., a main effect of self-esteem). However, this SE difference was expected to be greater following the generation of upward than downward counterfactual thoughts. The positive affective implications of upward comparisons (e.g., that it may well be possible to be in a better position in the future) are not as obvious as the positivity of downward comparisons. Consistent with past research (e.g., Brown et al., 1991), HSE persons are likely better able to identify and embrace the positive subtleties of upward counterfactuals. Thus, an ordinal interaction between SE and direction was hypothesized, such that HSE persons would always report more positive affect than LSE persons following the 
generation of counterfactuals, but that the magnitude of this SE difference would be greater in the case of upward as opposed to downward counterfactual generation. 
CHAPTER II

\section{EXPERMENT 1}


This first experiment constituted a preliminary experimental examination of the functional basis of counterfactual thinking. Specifically, the affective function was investigated using a paradigm based on retrospective self-reports of negative life events. Subjects were instructed to describe in writing a single event that they found especially negative or depressing. Subjects then recorded counterfactual alternatives to the event. The direction and structure of these counterfactual thoughts were manipulated. Subjects then made several ratings of their current mood, as well as perceptions of responsibility, controllability, and avoidability with respect to the event.

The principal objective of this preliminary experiment was simply to demonstrate the feasibility of a manipulation of counterfactual direction and structure. Although these variables have been employed as dependent measures, they have not been manipulated as independent variables in the published literature. The manipulation strategy used throughout the present program of research is based on asking subjects to fo ' is on and record exclusively one specific counterfactual subtype. That subjects presumably have no familiarity with these constructs necessitates well-worded experimental requests; poorly-worded, unclear, or misleading requests would engender ambiguous results. Two steps were taken to ensure the clarity of these requests. First, the skeleton of the request, in which "if only" thoughts are described in everyday parlance, was taken from my previous research that successfully evoked the recording of counterfactual thoughts by subjects (Roese \& Olson, 1993b, 1993c). Second, an example of each of the four 
counterfactual subtypes was included.

The manipulation checks were therefore a crucial part of this experiment. These checks were conducted in two ways. One check was nonparametric; the number of subjects was tabulated who, overall and within conditions, correctly recorded the counterfactual subtype requested of them. A parametric test was also used. By subtracting the total number of downward counterfactuals recorded from the total number of upward counterfactuals recorded by each subject, a univariate index of direction was derived for each subject. A similar index was calculated for structure. Analyzing these variables as a function of the direction and structure manipulations therefore represented a second check on the effectiveness of the manipulations. Any failures to manipulate the two focal variables could thus be identified within conditions, permitting repair and improvement before the commencement of the two principal experiments ( 2 and 3) in this research program.

Several hypotheses were derived on the basis of the foregoing theoretical explication. A main effect of counterfactual direction was predicted for the "ffective measures, such that more negative affect would result from the consideration of upward counterfactuals relative to consideration of downward counterfactuals. This prediction was made on two levels. First, the effect should be evident on a 5 -item mood scale designed to tap into globa! affective selfperceptions. Second, the effect was predicted to be particularly apparent on the single affect item poled by "disappointed" versus "relieved". Disappointment and relief are examples of the "counterfactual emotions" posited by Kahneman and Miller (1986). More recent research by Boninger, Gleicher, and Strathman (1992) has shown that counterfactual emotions can be differentiated not only 
conceptually but empirically from noncounterfactual emotional experiences (e.g., simply feeling "bad"). If, as these authors suggest, counterfactual emotions are those emotional experiences which by their very nature require a counterfactual judgment as a prerequisite (i.e., it is difficult to feel disappointment per se without the inference that things could have turned out better), then they should be particularly sensitive to any manipulation of counterfactual generation. The manipulation of counterfactual structure was not predicted to produce any shifts in mood ratings.

In order to replicate previous correlational demonstrations of a link between counterfactual direction and affect (e.g., Markman et al., 1993), an internal analysis was conducted in which the frequency of each type of counterfactual generated was correlated with the mood measures. I expected that the frequency of upward counterfactuals would be related to more negative affect, and that this relation would be particularly strong for the disappointed-relieved measure. Counterfactual structure was predicted to be unrelated to affect.

A secondary focus of this research was the possible moderating role of selfesteem in the effects of counterfactuals on perceptions of affect. HSE persons were expected to report more positive affect than LSE persons following counterfactual generation. Further, this SE difference was expected to be greater following upward than downward counterfactual generation.

Method

Subjects

Participants were 59 (22 men, 37 women) introductory psychology students attending the University of Western Ontario, randomly assigned to the four 
experimental conditions (with the constraint of equal cell sizes). Subjects participated in exchange for course credit.

\section{Materials and Procedure}

Upon entering the laboratory, subjects were seated in separate rooms. Although as many as four subjects were run per session, all completed their tasks privately and anonymously. After reading a short description of the study and signing a consent form, subjects were given booklets containing the experimental materials.

First, subjects were given a booklet containing the principal measures. The first page read as follows:

This study is part of an ongoing examination of the processes underlying coping with negative life events.

Please take a moment to think of a single event in the last year that happened to you and that was especially negative and/or disappointing. It should also directly involve at least one other person of your approximate age (for example, a fight between you and a friend).

In just a few words, please describe this event (keep in mind that this questionnaire is anonymous and confidential).

Subjects then briefly recorded the details of their event on the space provided on the rest of the page. Subjects tended to record intimate details of recent 
interpersonal strife. More specifically, $42 \%$ recorded a major interpersonal event (e.g., a fight with a friend, an arrest for drunk-driving, a boyfriend caught cheating), $25 \%$ recorded relatively less traumatic events (e.g., damage caused during a house paity, misunderstandings between friends), $22 \%$ described the break-up of a relationship, and $10 \%$ described a death.

Subjects then generated counterfactual alternatives to the event recorded, imagining what things could have been different to have changed the outcome. Half of the subjects were asked to record upward and half were asked to record downward counterfactuals. Further, and orthogonal to this factor, half were asked to record additive and half were asked to record subtractive counterfactuals. This yielded a $2 \times 2$ design with 4 resultant cells. Upward combined with additive counterfactuals were solicited as follows:

People often have thoughts like "if only ... " after these sorts of negative events, in that they can see how things might have turned out better. For example, a Toronto woman who recently sustained minor injuries when she was hit by a car told reporters, "If only 1 had looked down that street a second time, I would've been fine." Often, we wish we had done something to avoid a negative outcome. L: the space below, please list some specific actions that, in retrospect, could have been taken to have avoided the event you recalled.

Upward combined with subtractive counterfactuals were requested in the following manner: 
People often have thoughts like "if only ... " after these sorts of negative events, in that they can see how things might have turned out better. For example, a Toronto woman who recently sustained minor injuries when she was hit by a car told reporters, "If only I had NOT been in such a rush, I would've been fine." Often, we wish we hadn't done something that led to a negative outcome. In the space below, please list some specific actions that, in retrospect, should not have been done, which might have avoided the event you recalled.

Downard combined with additive counterfactuals were solicited as follows:

People often have thoughts like "well, at least ... " after these sorts of negative events. in that they can see how things might have turned out even worse. For example, a Toronto woman who recently sustained minor injuries when she was hit by a car told reporters, "At least no one moved me around after the accident, or it would've been a lot worse." Often, there are things that could have happened that would have made a negative outcome worse. In the space below, please list some specific actions that could have happened that would have made the event you recalled even worse.

Downward combined with subtractive counterfactuals were solicited as follows:

People often have thoughts like "well, at least ... " after these sorts of negative events, in that they can see how things might have turned out even 
worse. For example, a Toronto woman who recently sustained minor injuries when she was hit by car told reporters, "At least someone called an ambulance right away, or it would've been a lot worse." Often, negative outcomes could have been worse if certain actions had not been taken. In the space below, please list some specific actions that did occur in the event you recalled, and that would have made the event even worse if they had not occurred.

Subjects were free to record as many counterfactual thoughts as they wished. The remainder of the page was blank and intended for their use in recording these thoughts.

Next, all subjects made affective ratings that described "how thinking about this event makes you feel right now". The five bipolar ratings were depressedelated, negative-positive, unhappy-1’appy, hostile-agreeable, and disappointedrelieved. All ratings were made on 9-point scales ranging from -4 to +4 . Such ratings have been employed in previous mood research, and have been shown to be reliable and predictively valid (e.g., Esses, Haddock, \& Zanna, in press).

Subjects then made four attributional ratings. These ratings were of an exploratory nature, collected because theory and research have previously identified a relation between attributional and counterfactual thinking (e.g., Lipe. 1991; Wells \& Gavanski, 1989). Subjects rated (on 9-point scales) their perceived responsibility for the event described, the degree of responsibility others had for the event, the controllability of the event, and the avoidability of the event. Again, these ratings ranged from -4 to +4 . For ethical reasons, an attempt was made to place subjects in a more pleasant frame of mind. Thus, subjects then recalled a 
positive life experience and then made additional mood ratings.

Last, subjects completed the Texas Social Behavior Inventory (TSBI), short form A, a self-report measure focusing on the social aspects of dispositional selfesteem (Helmreich \& Stapp, 1974). The scale comprises 16 items, each answered on 9-point ratings. This instrument has been previously demonstrated to possess adequate psychometric properties (Blascovich \& Tomaka, 1991). The full scale is presented, along with the other materials used in this experiment, in Appendix B. The self-esteem scale was embedded within a series of other questionnaires of interest for separate research projects (one of these tasks was a pretest for Experiment 2; see Chapter III for details).

At the completion of each experimental session, subjects were fully debriefed and provided with a written description of this research and its goals.

\section{Results}

\section{Individual Difference Measures}

Scores on the TSBI self-esteem measure ranged from 55 to $114(M=84.2$; $M d=86$ ). This scale achieved adequate internal reliability (Cronbach's $\alpha=.80$ ); a median split was performed on these scores to produce HSE and LSE groups. Preliminary analyses in which sex of subject was a factor revealed no significant effects of this variable. It is therefore not discussed further.

\section{Manipulation Checks}

The nonparametric manipulation check was based on a simple dichotomous classification of whether or not subjects correctly followed instructions, recording the appropriate counterfactual type corresponding to their experimental 
conditions. All counterfactuals were classified as upward versus downward and additive versus subtractive.' Subjects were classified as "correct" if the number of counterfactuals corresponding to their condition exceeded all other noncorrespondent counterfactuals. Seventy-six percent of subjects (45 out of 59) were classified as correct, thus revealing a somewhat disappointing performance by the manipulational procedure. Of the 14 who were classified as incorrect, one was in the upward/additive condition, 7 were in the upward/subtractive condition, 2 were in the downward/additive condition, and 4 were in the downward/subtractive condition. Interpretation of the meaning of subtractive counterfactuals apparently involved more confusion (11 subjects) than did additive counterfactuals ( 3 subjects). ${ }^{2}$

The parametric test of the manipulations was based on two variables. Direction-dependent variable (Direction-DV) was defined as the number of downward counterfactuals subtracted from the number of upward counterfactuals

- A second rater, blind to the experimental conditions, coded a random sample of the responses of 20 subjects (34\% of the sample). Inter-rater agreement was high (99\% for direction, $97 \%$ for structure). Examples of each subtype appear in Appendix A.

2 The affective hypothesis focuses on more positive feelings resulting from downward counterfactuals. If, however, the direction manipulation also alters the focus of counterfactual thoughts (i.e., internally vs. externally derived acts), results may be confounded. Specifically, if downward counterfactuals typically involve external attributions, whereas upward counterfactuals involve internal attributions, then downward counterfactuals may elicit more positive affect because blame for failure has been attributed externally (e.g., McFarland \& Ross, 1982; Roese \& Olson, 1993b). To examine this possibility, counterfactual responses were coded for internal vs. external focus. Neither direction nor structure influenced internality vs. externality of counterfactuals recorded. Moreover, an analysis of covariance (ANCOVA), with the repeated measures covariate of internal vs. external focus, did not alter the affect conclusions presented in the text. In Experiments 2 and 3, subjects were orally requested to record only internally-derived actions. All complied. 
recorded in total by each subject. Hence, more positive values indicated greater frequency of upward counterfactuals, whereas more negative values indicated more frequent downward counterfactuals. Similarly, structure-DV was defined as the additive minus the subtractive counterfactuals recorded by each subject. A 2 (direction) $\times 2$ (structure) $\times 2(\mathrm{SE})$ analysis of variance (ANOVA), using direction-DV as the dependent measure, revealed the expected main effect for direction, $F(1,51)=99.84, p<.001$. Upward counterfactuals were more frequent in the upward condition and downward counterfactuals were more frequent in the downward condition ( $M \mathrm{~s}=3.07,-2.21$, respectively). The manipulation of structure had no effect on this measure $(F=.17, \mathrm{~ns})$, and no other effects were reliable. Another $2 \times 2 \times 2$ ANOVA performed on structureDV revealed the predicted main effect of structure, $F(1,51)=32 .(0), p<.001$. with the means falling in the expected directions $(M=2.00$ in the additive condition; $M=-.90$ in the subtractive condition). As expected, the direction manipulation had no effect on this measure $(F=1.57, \mathrm{~ns})$. However, there was a significant main effect for self-esteem, $F(1,51)=4.51, p<.05$. HSE subjects recorded more additive counterfactuals $(M=1.24)$ than did LSE subjects $(M=$ $-.07)$. No other effects were reliable in this analysis. ${ }^{3}$

'If some counterfactual subtypes are more available than others, then analyses of the impact of direction and structure could be confounded with the total number of counterfactual thoughts recorded. To rule out this possibility, the principal analyses presented in all three experiments were reconducted as ANCOVAs, in which the total number of counterfactuals recorded was the covariate. In no instance was the latter variable a significant covariate, and all effects remained significant, with one exception that fell to a marginal level. Thus, the principal statistical conclusions were confirmed using ANCOVAs. 
Affect

The 14 subjects who incorrectly responded to the counterfactual request were dropped from the present analyses of affect to permit a more sensitive test of the hypotheses. A $2 \times 2 \times 2$ ANOVA was first performed on the mood scale (i.e., the summary index of the 5 mood ratings). ${ }^{4}$ The mood scale was found to be reliable (Cronbach's $\alpha=.83$ ). There were no significant main or interaction effects. Thus, the hypothesis that counterfactual direction would produce effects on a global mood measure was not supported.

Another $2 \times 2 \times 2$ ANOVA was used to examine the single disappointedrelieved rating. As predicted, the main effect for direction was significant $F(1,37)$ $=4.63, p<.05$. Consideration of upward counterfactuals resulted in greater perceptions of disappointment $(M=2.86)$ than did consideration of downward alternatives $(M=4.30)$. No other effects were significant in this analysis. The means of both affect measures within levels of the manipulated variables are presented in Table 1.

\section{Attributional Ratings}

The attributional ratings were analysed using $2 \times 2 \times 2$ ANOVAS. These analyses revealed no reliable findings; hence they are not presented in detail.'

${ }^{4}$ Ratings were made on scales ranging from -4 to +4 . For clarity of presentation, these values were transformed to scales ranging from 1 to 9 . This practice was employed in all 3 experiments.

'For both affective and attributional ratings, ANOVAs conducted on the full sample of 59 revealed no significant effects. 
Table 1

Summary of within-cell means of the affective measures

\begin{tabular}{lcccc}
\hline & \multicolumn{4}{c}{ Condition } \\
\cline { 2 - 5 } Measure & $\begin{array}{l}\text { Upward/ } \\
\text { Additive }\end{array}$ & $\begin{array}{l}\text { Upward/ } \\
\text { Subtractive }\end{array}$ & $\begin{array}{l}\text { Downward/ } \\
\text { Additive }\end{array}$ & $\begin{array}{c}\text { Downward/ } \\
\text { Subtractive }\end{array}$ \\
\hline Mood Scale & 4.07 & 3.10 & 4.45 & 3.78 \\
Disappointed/Relieved & 3.21 & 2.25 & 4.77 & 3.70 \\
$n$ & 14 & 8 & 13 & 10 \\
\hline
\end{tabular}

Note. $n=$ number of subjects per cell. Means are weighted by the cell size. Higher scores indicate more positive affect. 


\section{Correlations}

Correlational analyses between direction-DV, structure-DV, and the mood measures were also conducted. The first two variables are the same as those used in the parametric test of the manipulations. Table 2 displays the relevant correlations. This "internal analysis" supports the conclusions from the disappointed-relieved ANOVA. Structure was unrelated to mood, whether the latter was operationalized using the mood scale, or the more specific disappointedrelieved measure. Direction was negatively related to the disappointed-relieved measure, $r(43)=-.44, p<.001$, such that more frequent consideration of upward counterfactuals covaried with more negative, disappointed affective states. This relation is weaker but still significant when the mood scale was used as the index of affect, $r(43)=-.31, p<.(12$.

In correlational analyses of the attributional ratings, again, no reliable effects emerged. An exception was noted for the correlation between outcome avoidability and the affect measures. Subjects perceiving the outcome to be avoidable tended to report more negative affect, $r(41)=-.45$ (disappointed/relieved), $r(41)=-.36(\operatorname{mood} s c a l e)$, both $p s<.01$.

\section{Discussion}

The main goal of this first experiment was achieved. The manipulation of counterfactual direction and structure was shown to be feasible. Manipulation check data indicated that subjects were able to generate upward versus downward counterfactuals in response to written requests with reasonable alacrity. However, the manipulation of additive versus subtractive counterfactuals was not as 
Table 2

Correlations between counterfactual direction. structure, affect, and attributional ratings

\begin{tabular}{|c|c|c|c|c|c|c|c|}
\hline & ST & MS & DR & $\mathbf{R} \mathbf{1}$ & $\mathbf{R 2}$ & $\mathbf{R 3}$ & R4 \\
\hline Direction-DV & .24 & $-.31^{*}$ & $-.44^{* *}$ & -.19 & -.06 & -.18 & .19 \\
\hline Structure-DV & & .11 & .06 & -.02 & .00 & .14 & .06 \\
\hline Mood Scale & & & $.80^{* *}$ & -.07 & .15 & .10 & $-.36^{* *}$ \\
\hline \multicolumn{2}{|c|}{ Disappointed/Relieved } & & & -.06 & .15 & .10 & $-.45^{* *}$ \\
\hline R1 & & & & & -.19 & $.51^{* *}$ & $.32^{*}$ \\
\hline R2 & & & & & & -.16 & -.21 \\
\hline R3 & & & & & & & .17 \\
\hline
\end{tabular}

Note. For direction-DV, higher numbers indicate more frequent upward counterfactuals; ST = Structure-DV (higher numbers indicate more frequent additive counterfactuals); $D R=$ disappointed-relieved affect measure; $M S=$ mood scale (for both affective measures, higher numbers indicate more positive affect): $R 1$ = rating of personal responsibility; $\mathbf{R} 2$ = rating of responsibility of others; $\mathbf{R 3}=$ rating of outcome controllability; $\mathbf{R 4}=$ rating of outcome avoidability (for all attributional ratings, higher numbers indicate more of the specified quality). For correlations between counterfactual and affect measures, $N$ $=45$. For correlations involving attribution ratings, $N=43$, due to missing data. ${ }^{*} p<.05 ;{ }^{* *} p<.01$. 
successful. When based on nonparametric criteria, the manipulation check showed that almost a quarter of the subjects in the present study failed to respond correctly to the manipulation. Moreover, this failure appeared to stem from a misunderstanding of the meaning of subtractive counterfactuals, particularly of the upward/subtractive subtype. Thus, improvement of the questionnaire wording within this cell was undertaken (see Experiment 2). Nevertheless, this first experiment provides crucial confirmation of the feasibility of manipulations of direction and structure, thus providing the necessary methodological grounding for the subsequent two experiments reported here.

The findings of this first study supported the hypothesis that counterfactuals can causally influence affect. Specifically, the generation of upward as opposed to downward counterfactuals was more likely to result in negative affect, when affect was defined in terms of disappointment versus relief. However, direction did not influence scores on the global mond scale. As expected, the manipulation of counterfactual structure did not intluence affect. regardless of how the latter was defined.

Previous research has demonstrated a significant relation between dire stion and affect (e.g., Markman et al., 1993). That is, upward counterfactuals tend to co-occur with negative affect. This negative correlational finding was in fact replicated in the present study, using an internal analysis of total number of counterfactuals generated and the global affect measure. Because these findings are correlational. it is unclear whether counterfactual direction caused changes in affective perceptions, or if the reverse causal patter, was the case. The present findings, however, also indicate that counterfactual direction can causally influence affect. Subjects induced to generate upward counterfactuals later reported more 
negative affect than those induced to generate downward counterfactuals. However, this finding is weakened somewhat by the fact that it was evident only on a very specific measure of affect (the disappointed-relieved measure), and not on a more global measure of affect. Indeed, it could even be argued that this finding is tautological, in that disappointment and relief are by their very nature subexamples of counterfactual inference. Thus, the demonstration of direction effects on the mood scale remains a crucial goal for Experiment 2.

There may be at least three reasons why a significant effect was not found using the mood scale. The first is that the mood measure was simply not sensitive to real shifts in affective experience. Given the adequate reliability of the affect scale evidenced in the present sample (as assessed using an index of internal consistency), coupled with previous successful applications (e.g., Esses, Haddock, \& Zanna, in press), this possibility does not appear likely. A second reason is that the manipulation was too weak to exert an effect on any but the most sensitive of measures (i.e., the disappointed-relieved measure). Third, and related to this, the elimination of subjects for whom the manipulation was unsuccessful reduced the sample size and hence the power to detect meaningful differences. Very likely a combination of the latter two possibilities accounts for the failure to detect counterfactual effects using the mood scale. For the second study, both modifications to the manipulation procedure (see below) and a larger sample size should permit a more effective test of these hypotheses. 


\section{CHAPTER III}

\section{EXPERIMENT 2}


In terms of documenting of an affective function served by counterfactuals, the second experiment was intended to be both an improvement and extension of the first experiment. The problems with the manipulations in Experiment 1 were addressed with procedural revisions. Affect again constituted a dependent measure, allowing a test of the affective function hypothesis. Also, the preparative function was examined using behavioural intention ratings as dependent measures.

Two changes were made to the manipulation procedure. The examples used to illustrate the four types of counterfactuals were modified so that all four reflected active, internally-driven actions. Second, the manipulation was accompanied by a short oral reminder to subjects that they should only write down counterfactuals consistent with the request, and not to write down counterfactuals of any other types. This change, co:ipled with a larger sample size, was expected to permit a demonstration of the affective function of counterfactual thoughts. Thus, consideration of upward counterfactuals was expected to result in more negative affect than consideration of downward counterfactuals. This effect was expected on both the global affect measure as well as the disappointed-relieved measure.

A second goal of this experiment was to provide a demonstration of the preparative function of counterfactual thinking. The hypothesized mechanism linking counterfactuals to behaviour is the formation of plans from counterfactual alternatives to past actions. Specifically, the conversion of counterfactuals into prefactuals, that is, into conditional statements regarding the consequences of 
performing certain actions in the future, might result in future improvement. Previous evidence has shown that the mere consideration of prefactuals increases intentions to perform relevant behaviours, and as well increases the likelihood of actually performing such relevant behaviours (e.g., Anderson, 1983; Gregory et al., 1982; Sherman et al., 1981). To the extent that such behaviours facilitate performance, then counterfactuals of certain types may be said to improve causally performance. The present study was designed to assess the impact of counterfactual thinking on behavioural intentions, as assessed by ratings of intentions to perform success-facilitating behaviours. The mediation of this link by the consideration of prefactuals that are fabricated from counterfactuals was not directly assessed.

Subjects were asked to provide retrospective self-reports of recent negative outcomes. In contrast to the first experiment, however, these outcomes were more specific, focusing explicitly on a recent performance on an examination that was poor and therefore disappointing. Subjects were directed to generate counterfactuals based on the $2 \times 2$ manipulation of direction and structure. As noted, a principal dependent measure was intention ratings regarding the performance of 6 behaviours facilitative of academic success. These 6 behaviours were identified on the basis of a pretest completed by a separate sample of university students (subjects in Experiment 1 completed this pretest following the completion of the main experimental procedures); these behaviours therefore represented the actions most frequently generated spontaneously by students when asked to consider how to improve their grades.

These behaviours were of two types. Commissional behaviours were those actions that should be taken (e.g., going to visit professors for extra help), whereas 
omissional behaviours were those that should not be taken (e.g., not watching TV before an exam). Consistent with the foregoing theoretical framework, it was hypothesized that there would be a main effect for direction on both commissional and omissional behavioural intentions, such that upward counterfactuals would result in heightened intentions to perform both. As discussed in the introduction, upward as opposed to downward counterfactuals may be more easily converted into prefactuals, which in turn may increase intentions to perform relevant behaviours facilitative of success (see Figure 1).

It was also predicted that the structure dimension would play a causal role, such that additive counterfactuals would increase intentions to perform commissional behaviours. However, predictions regarding the influence of additions versus subtractions on omissional behavioural intentions were less clear. The prediction most consistent with the foregoing theoretical position would be that additive counterfactuals would heighten these intentions as well, relative to counterfactual subtractions. Because additive counterfactuals are more specific and more creative, perhaps as a result of more effortful cognitive processing, generation of them might facilitate the identification of more useful and incisive behavioural strategies, relative to consideration of subtractive counterfactuals. Further, this effect might occur independently of how the behavioural strategies are phrased grammatically. Relative to those considering subtractive possibilities, those considering additive counterfactuals may therefore be more likely to note in the first place the actional significance of any behavioural strategy, to convert it into a plan, and to implement that plan. Alternatively, subtractive courterfactuals might be predicted to heighten intentions to perform omissional behaviours. The logic behind this prediction is based on the notion that counterfactual additions 
should facilitate the formation of intentions that are also based on additions (i.e., on the addition of new antecedent actions). Counterfactual subtractions, on the other hand, identify what should not have been done, hence they might be predicted to influence intentions that are also rooted in avoiding certain actions.

Examination of the independent impact of the direction and structure variables offers a unique test of the functional effects of counterfactual thinking, but their interpretation may be somewhat ambiguous. Specifically, if a main effect of direction on affect is found, it is unclear whether it is the positive affectenhancing influence of downward counterfactuals, the affect-depressing influence of upward counterfactuals, or a combination of both that is responsible for the observed group differences. A baseline measure of affect would provide an appropriate solution to this ambiguity. Accordingly, a control group was included in this experiment, one in which subjects were run through exactly the same procedure as the four experimental conditions, with the exception that counterfactual thoughts were neither solicited nor mentioned. Not only can such a control group illuminate the specific nature of any main effects of the two independent variables, but it permits a test of the mere impact of counterfactual generation per se. That is, the effect of simply generating any counterfactual can be examined by comparing the weighted mean of the four experimental conditions to the control condition. In using the control group to assess the mere effect of counterfactual generation, it can be predicted that merely considering a counterfactual will result in heightened behavioural intentions of performing both commissional and omissional facilitative behaviours. Such a difference would not be expected for the affect measures. That is, because upward counterfactuals may create negative affect and downward counterfactuals may create positive affect, 
relative to the control condition, the two experimental conditions combined would not be expected to differ from controls.

Self-esteem was also assessed in the present experiment. Although this individual difference variable did not significantly moderate any of the affective findings in Experiment 1, this may have been due to the previously described problems with the manipulation and the sample size. Thus, I again predicted that HSE persons would report more positive affect than LSE persons overall, but that the magnitude of this SE difference would be greater for upward relative to downward counterfactual generation.

\section{Method}

\section{Subjects}

Participants were 90 (46 women, 44 men) introductory psychology students attending the University of Western Ontario, randomly assigned to the five experimental conditions, with the constraint of roughly equal cell sizes. All participants received course credit in exchange for participation. Four subjects were eliminated from the original sample of 94 because of failure to complete fully all experimental materials.

\section{Materials and Procedure}

Subjects were tested in groups of approximately fifteen to twenty. Upon entering the laboratory, subjects seated themselves one to a table, read a short description of the procedure, and signed consent forms.

Subjects were first given a booklet containing the principal measures. First. subjects were asked to reflect on a recent performance on an examination that 
they considered disappointing:

This study is part of an ongoing examination of the processes underlying coping with negative life events. Please take a moment to think of an exam you wrote recently (e.g., in the last year) that went especially poorly, and that you were especially disappointed with. In just a few words, please provide a few details about the exam (e.g., the course it was in, when you wrote it, etc.).

Subjects then briefly recorded the details of the event recalled on the space provided on the rest of the page. With one exception, all subjects complied without apparent difficulty. One subject did, however, complain to the experimenter that she was unable to think of a recent disappointing exam performance. She was therefore instructed to think back as far as possible to retrieve an appropriate memory (this turned out to be seven years ago).

As in Experiment 1, subjects then generated counterfactual alternatives to the event recorded, imagining what things could have been different to have changed the outcome. Half of the subjects were asked to record upward and half were asked to record downward counterfactuals. Further, and orthogonal to this factor, half were asked to record additive and half were asked to record subtractive counterfactuals. This yielded a $2 \times 2$ design with 4 cells. Upward combined with additive counterfactuals were solicited as follows:

- The examples in ine solicitations were modified so that all four focused on internally-derived actions. Thus, the example of a downward/additive counterfactual was changed from "At least no one moved me around ..." to "At least I didn't try to move around". 
People often have thoughts like "if only ... " after negative events, in that they can see how things might have turned out better. For example, a Toronto woman who recently sustained minor injuries when she was hit by a car told reporters, "If only I had looked down that street a second time, I would've been tine." Often, we wish we had done something to avoid a negative outcome. In the space below, please list some specific actions that, in retrospect, could have been taken to have improved your exam score.

Upward combined with subtractive counterfactuals were requested in the following manner:

People often have thoughts like "if only ... " after negative events, in that they can see how things might have turned out better. For example, a Toronto woman who recently sustained minor injuri ss when she was hit by a car told reporters, "If only I had NOT been in such a rush, I would've been fine." Often, we wish we hadn't done something that led to a negative outcome. In the space below, please list some specific actions that, in retrospect, should not have been done, which might have improved your exam score.

Downward combined with additive counterfactuals were solicited as follows:

People often have thoughts like "well, at least ..." after negative events, in that they can see how things might have turned out even worse. For 
example, a Toronto woman who recently sustained minor injuries when she was hit by a car told reporters, "At least I didn't try to move around after the accident, or it would've been a lot worse." Often, there are things that could have happened that would have made a negative outcome worse. In the space below, please list some specific actions that you could have done that would have made your exam score even worse.

Downward combined with subtractive counterfactuals were solicited as follows:

People often have thoughts like "well, at least ... " after negative events, in that they can see how things might have turned out even worse. For example, a Toronto woman who recently sustained minor injuries when she was hit by car told reporters, "At least I had my medic-alert bracelet on, or it would've been a lot worse." Often, negative outcomes could have been worse if certain actions had not been taken. In the space below, please list some specific actions that you did before the exam, and that would have made your exam score even worse if you had not done them.

These written solicitations were accompanied by an oral admonishment to record only the counterfactual subtype specified. Subjects were free to record as many counterfactual thoughts as they desired (an entire page was left blank for this purpose). A fifth, control condition was also used. Subjects in this condition completed tasks identical to the other four, with the single exception that they generated no counterfactual thoughts and heard no mention of "if only" ideas until the debriefing at the end of the experimental session. These subjects were run in 
groups separate from the four experimental conditions.

Subjects next made affective ratings in response to the request, "please indicate how thinking about your exam score makes you feel right now by circling a number on each of the following scales." Eight bipolar items appeared in the following order: disappointed-relieved, concerned-unconcerned, depressed-elated, tired-awake, negative-positive, anxious-calm, unhappy-happy, hostile-agreeable. All ratings were made on 9-point scales ranging from -4 to +4 . Two changes in the format from Experiment 1 were made. First, the disappointed-relieved item was placed first rather than last, to ensure a more sensitive test of the relevant hypothesis. Second, several filler ratings were placed within the crucial mood ratings (concerned-unconcerned, tired-awake, anxious-calm) in an attempt to lessen the experimental demand that may emerge from an obvious mood rating requested directly following the counterfactual task. As in Experiment 1, the five mood ratings were summed to yield a composite mood scale.

Subjects then rated their perceived responsibility, the responsibility of others, the controllability of the event, and the avoidability of the event. These ratings were also made on 9-point scales ranging from -4 to +4 .

Subjects then completed the intention ratings regarding the performance of 6 behaviours that could presumably improve academic performance in university. These behaviours were selected on the basis of a pretest given to subjects in Experiment 1 following the completion of the principal procedures of that study. The pretest consisted of a single sheet of paper with the following instructions:

This is a "pretest" for a future study that is now in preparation. Imagine that you have done very poorly on an important exam. Assuming that you 
are highly motivated to do better in the future, what spewati actions would you take to improve your chances of success?

Four spaces were provided so that subjects could record four different behaviours. All 59 subjects complied. A total of 18 prototypic behaviours were identified on the basis of subjects' responses. The six most frequently recorded behaviours were rewritten so that 3 would constitute commissional behaviours and 3 would constitute omissional behaviours. The three commissional statements were: "Studying/reviewing your notes three or more nights during the week"; "Regularly seeing your professors to ask questions and discuss problem areas"; "Trying to attend every lecture during the school year". The three omissional statements were: "No drinking or partying for one week before exams"; "No watching TV on at least three nights during the week before exams": "Not going away on the weekend before the week of an exam". St: jects in the present experiment made 9-point ratings ranging from -4 to +4 , anchored by "not at all likely to do this" and "extremely likely to do this". The format of these questions was based loosely on that of Anderson (1983). As a more global index of expectancy, subjects also indicated what grade average (expressed as a percentage) they expected to achieve at the end of the current academic year.

For ethical reasons, an attempt was made to place subjects in a more pleasant frame of mind. Thus, subjects then recalled a positive life experience and then made additional mood ratings.

The last phase of the procedure involved the completion of two individual difference scales, with their respective items interspersed throughout a single questionnaire. The items were taken from short form A of the Texas Social 
Behavior Inventory (TSBI) and from Paulhus' (1984) social desirability (SD) scale. The latter scale was included to provide a test of the possible influence of social desirability biases on any findings. Specifically, items 3, 5, 6, 7, 9, and 10 from the Impression Management subscale were adapted for use in both this and the subsequent experiment. In his factor analyses, these items loaded heavily onto a social desirability factor centring on deliberate, strategic self-presentational enhancement, as opposed to more unconscious or unwitting forms of socially desirable responding (Paulhus, 1984). The items from this social desirability scale are presented, along with the other materials used in this experiment, in Appendix C. Last, subjects completed a post-experimental questionnaire designed to probe their awareness of the principal hypotheses.'

At the completion of each experimental session, subjects were fully debriefed and provided with a written description of this research and its goals.

\section{Results}

\section{Individual Difference Measures}

Scores on the TSBI self-esteem measure ranged from 59 to $140(M=97.9$; $M d=99$ ). This scale achieved adequate internal reliability (Cronbach's $\alpha=.82$ ). A median split was performed on the SE scores to produce HSE and LSE groups. Scores on the social desirability measure ranged from 14 to 65 ( $M=42.65 ; M d=$

The post-experimental questionnaires employed in both Experiments 2 and 3 revealed no evidence for awareness of the hypotheses in any of the subjects. Although some subjects agreed with the suggestion that "if only" thoughts are somehow related to both feelings and performance, none were able to articulate these relations spontaneously, or in terms of differences in types of counterfactuals. 
43). This measure, however, was much less internally reliable $(\alpha=.65)$.

Nevertheless, a median split was performed on the SD scores to produce HSD and LSD groups.

Preliminary analyses indicated that SD was rarely a significant factor. Thus, the analyses that follow are presented without SD as a factor. For those few exceptions in which SD was significant as a main or interaction effect, complete ANOVA information regarding this factor accompanies the discussion of the principal findings. Sex of subject was not significant as a main effect or as part of interaction effects, and thus is not discussed further.

\section{Manipulation Checks}

The nonparametric manipulation check was, as in the first exneriment, based on a simple dichotomous classification of whether or not subjects correctly followed instructions, recording the appropriate counterfactual type corresponding to their experimental conditions. ${ }^{8}$ Subjects were classified as "correct" if the number of counterfactuals corresponding to their condition exceeded all other noncorrespondent counterfactuals. All but 5 of 90 correctly followed isstructions, thus yielding a $94 \%$ success rate for the manipulation. Of the 5 who failed to follow instructions, one was in the upward/subtractive condition, 2 were in the downward/additive conditior. and 2 were in the downward/subtractive condition.

* The classification of counterfactuals according to direction and structure was undertaken by a second coder who was blind to the experimental conditions. The responses of 25 subjects ( $27 \%$ of the sample) were randomly selected for this purpose. Inter-rater agreement was high ( $100 \%$ for direction, $90 \%$ for structure). Examples of each subtype appear in Appendix A. 
These 5 subjects were eliminated from all subsequent analyses."

The parametric test of the manipulations was based on the same two variables defined in Experiment 1, direction-DV and structure-DV. A 2 (direction) $\times 2$ (structure) $\times 2(\mathrm{SE})$ analysis of variance (ANOVA), using direction-DV as the dependent measure, revealed the expected main effect for direction. $F(1,62)=295.38, p<.001$. Upward counterfactuals were more frequent in the upward condition and downward counterfactuals were more frequent in the downward condition $(M \mathrm{~s}=3.69 .-3.31$. respectively). A significant interaction between direction and SE was also found, $F(1,62)=5.07, p<.05$. Specifically. HSE and LSE people did not differ in the number of counterfactuals recorded in the downward condition ( $M \mathrm{~s}=-3.50 \mathrm{vs}-3.00$, respectively), but HSE people were able to generate significantly mor: upward counterfactuals $(M=$ 4.67) than were LSE people $(M=3.17) . l(62)=2.47 . p<.05$. The manupulation of structure had no effect on direction-D ${ }^{\prime}(F=.31$. ns $)$, and no other effects were reliable.

Another $2 \times 2 \times 2$ ANOVA performed on structure-DV revealed the predicted main efiect of structure, $F(1,62)=254.51, p<.(001$. with the means falling in the expected directions $(M=2.76$ in the additive condition; $M=-3.25$ in the subtractive condition). As expected, the direction manipulation had no effect on this measure ( $F=1.01$, ns). Unlike Experiment 1, the main effect for selfesteem was not significant $(F=.08 . \mathrm{ns})$. No other effects were reliable in this analysis.

"Analyses conducted on the full sample of 90 yielded the same conclusions presented in the text. with the exception that the main effect of direction on the affect scale was only marginally significar: $(p=.07)$ 
Affect

Mood scale. A $2 \times 2 \times 2$ ANOVA was first performed on the mood scale (comprising the 5 mood items). The mood scale was found to be somewhat less reliable than in Experiment 1 (Cronbach's $\alpha=.76$ ). As predicted but unlike Experiment 1, there was a significant main effect for direction, $F(1,62)=5.47, p$ $<$.03. Consideration of upward counterfactuals resulted in more negative affect $(M=3.75)$ than did consideration of downward counterfactuals $(M=4.37)$.

Thus, the main prediction for the affective function was supported. See Figure 2 for a depiction of this effect (higher scores in this figure indicate more positive affect).

Unexpectedly, the interaction between structure and SE was significant, $F(1,62)=4.23, p<.05$. For HSE subjects. crnsideration of additive counterfactuals resulted in more positive affect $(M=4.59)$ than consideration of subtractive counterfactuals $(M=3.65), t(62)=2.42, p<.05$, whereas for LSE subjects, this difference was not significant $(M \mathrm{~s}=3.84$ vs 4.12$), t(62)=.76$, ns. There were no other significant main or interaction effects in this analysis.

As noted in the introduction to this experiment, the inclusion of a nocounterfactual control group permits a test of the nature of the effect of direction on affect. Contrasts were employed to examine this effect. based on the mean square error from an omnibus oneway ANOVA in which all 5 conditions served as levels. The main effect was significant from this analysis, $F(4,80)=4.46, p<.01$. First, the mean mood score for the upward counterfactual condition ( $M=3.75$ ) was contrasted with the control condition $(M=3.12)$. The means did not differ 
Figure 2

Effect of countcifactual direction on global affect scores

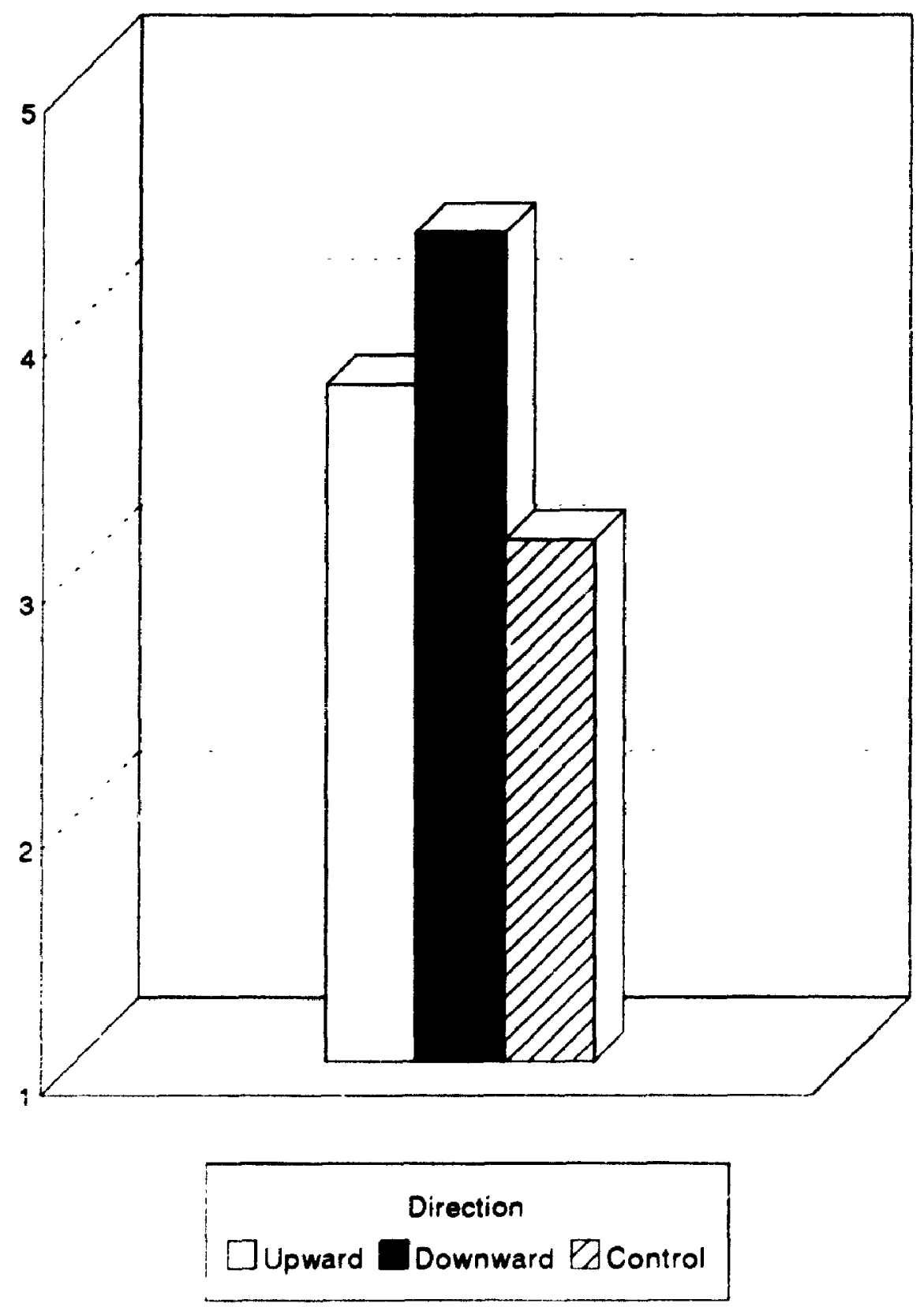


reliably between these two groups, $t(80)=1.84$, ns. ${ }^{10}$ However, subjects in the downward counterfactual conditions reported more positive affect $(M=4.37)$ than did those in the control condition, $\|(\cdots)=3.67, p<.001$. Thus, the main effect of direction on affect is not based on any affect-depressing influence of upward counterfactuals, but mainly on the positive affect-enhancing effects of downward counterfactual consideration. Because the $2 \times 2 \times 2$ ANOVA did not reveal a significant effect of structure on the mood scale, this variable was not assessed in relation to the control group. A contrast in which the means of the four experimental conditions, weighted by their cell sizes, were compared to that of the control condition, was significant $(M \mathrm{~s}=4.06$ vs 3.12 , respectively $) \cdot(80)=2.98, p$ $<.01$. Thus, the mere consideration of counterfactuals resulted in more positive affect (although it was clearly the downward counterfactuals that achieved this effect).

Disappointed-relieved. Another $2 \times 2 \times 2$ ANOVA was used to examine the single disappointed-relieved rating. As predicted, the main effect for direction was significant $F(1,62)=5.02, p<.05$. Consideration of upward counterfactuals resulted in greater perceptions of disappointment $(M=2.49)$ than did consideration of downward alternatives $(M=3.57)$. No other effects were significant in this analysis.

The control group analysis yielded findings that closely paralleled those of the mood scale. The main effect from the omnibus oneway ANOVA in which all 5 conditions served as levels was significant, $F(4,80)=3.78 . p<.01$. The mean

10 All contrasts reported in this dissertation are two-tailed, and were conducted using the mean square error from the relevant ANOVA (e.g., Carmer \& Swanson, 1973). 
disappointed-relieved ratings did not differ reliably between the upward counterfactual conditions $(M=2.49)$ and the control group $(M=1.93), t(80)=$ 1.04, ns. Subjects in the downward counterfactual conditions reported more relief $(M=3.57)$ than did those in the control condition, $1(80)=3.06, p<.01$. The contrast between the four experimental conditions $(M=3.03)$ ard the control condition was also significant, $t(80)=2.22, p<.05$.

The means for the affect measures within levels of the manipulated variables are shown in Table 3.

\section{Intention Ratings}

Commissional intention ratings. Scores from the 3 commissional intention ratings were correlated (mean $r[83]=.27, p<.05$ ) and hence averaged to yield a single commissional intention index (CII). A $2 \times 2 \times 2$ ANOVA performed on this variable revealed a significant main effect for direction, $F(1,62)=4.07, p<$ .05. Subjects directed to generate upward counterfactuals reported higher intentions to perform facilitative behaviours $(M=6.35)$ than those who generated downward counterfactuals $(M=5.47)$. This effect is depicted in Figure 3 (higher scores in this figure indicate greater intentions). No other effects were significant in this analysis.

The control group analysis indicated that, in contrast to the findings for affect, the direction effect was due to the impact of upward counterfactuals. The main effect from the omnibus oneway ANOVA in which all 5 conditions served as levels was not significant. however, $F(4,80)=1.83, p=.13$. Subjects who generated upward counterfactuals reported higher intentions $(M=6.35)$ than did control subjects $(M=5.11), t(8(1)=2.23, p<.05$. Subjects who generated 
Figure 3

Effect of counterfactual direction on commissional intention ratings

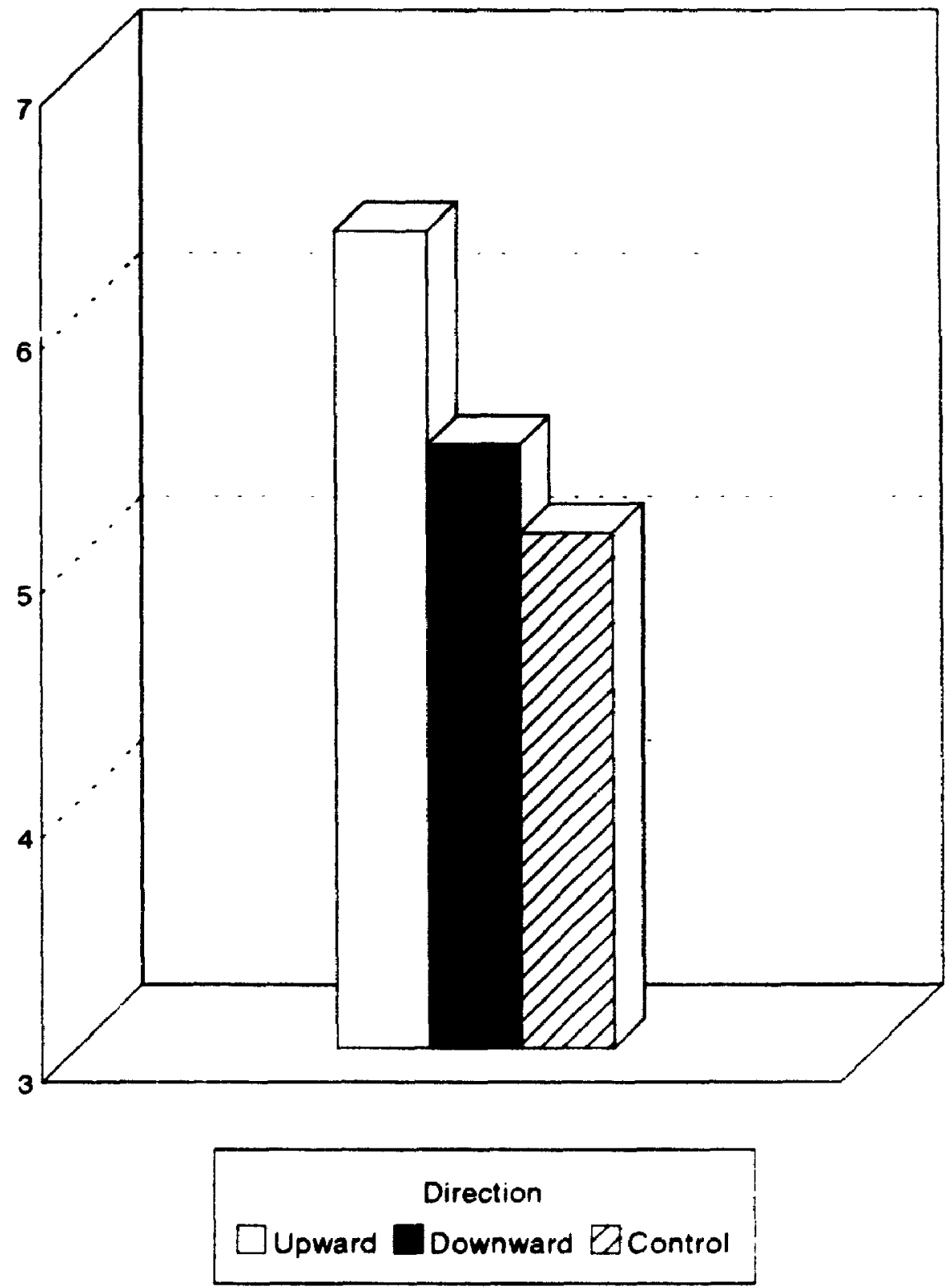


Table 3

Summary of within-cell means of the principal dependent measures

\begin{tabular}{lccccc}
\hline & \multicolumn{5}{c}{ Condition } \\
\cline { 2 - 6 } Measure & $\begin{array}{l}\text { Upward/ } \\
\text { Additive }\end{array}$ & $\begin{array}{l}\text { Upward/ } \\
\text { Subtractive }\end{array}$ & $\begin{array}{l}\text { Downward/ } \\
\text { Additive }\end{array}$ & $\begin{array}{l}\text { Downward/ } \\
\text { Subtractive }\end{array}$ & Control \\
\hline Mood Scale & 3.74 & 3.75 & 4.74 & 4.05 & 3.12 \\
Dis/Rel & 2.78 & 2.18 & 4.06 & 3.16 & 1.93 \\
CII & 6.17 & 6.55 & 5.69 & 5.30 & 5.11 \\
OII & 7.33 & 7.33 & 6.31 & 6.35 & 7.11 \\
Grade & 71.8 & 72.5 & 75.9 & 77.9 & 72.3 \\
\multicolumn{1}{c}{$\mathrm{n}$} & 18 & 17 & 16 & 19 & 15 \\
\hline
\end{tabular}

Note. Dis/Rel $=$ disappointed-relieved rating (for both affective measures, higher numbers indicate more positive affect): $\mathrm{CII}=$ commissional intention index; OII $=$ omissional intention index (higher scores mdicate greater intentions); $n=$ number of subjects per cell. 
downward counterfactuals did not differ in their intentions $(M=5.47)$ from control subjects, $I(80)=.66$, ns. The contrast between the four experimental conditions $(M=5.91)$ and the control condition was not significant, $t(80)=1.56$.

Omissional intention ratings. Scores from the 3 omissional intention ratings were correlated (mean $r[83]=.30, p<.05$ ) and hence averaged to yield a single omissional intention index (OII). A $2 \times 2 \times 2$ ANOVA performed on this variable indicated that the main effect for direction was only marginally significant, $F(1,62)$ $=3.50, p=.066$. Subjects generating upward counterfactuals reported greater intentions $(M=7.33)$ than those generating downward counterfactuals $(M=$ 6.33). The main effect for structure was not signiitcant, $F(1,62)=.01$, but this variable interacted significantly with SE, $\mathrm{F}(1,62)=6.79, p<.05$. For LSE subjects, consideration of additive versus subtractive counterfactuals resulted in equivalent intentions $(M s=6.71,7.67$, respectively $), t(62)=1.61, \mathrm{~ns}$. For HSE subjects, in contrast, the additive structure resulted in greater intentions to engage in omissi nal behaviours $(M=7.00)$ than did subtractive structures $(M=5.75)$, $t(62)=1.98, p<.05$. It is possible, however, that the Oll is somewhat less sensitive than the CII: in preliminiry analyses involving the SD variable, significant effects were obtained only with the former index. Using a 2 (direction) $\times 2$ (structure) $\times 2(\mathrm{SD})$ ANOVA, a significant main effect of SD on the Oll was found, $F(1,62)=6.34, p<.05$, such that HSD persons reported greater intentions $(M=7.37)$ than did LSD persons $(M=6.23) .{ }^{.1}$

"Both the CII and OII data can also be analysed using multivariate analyses of variance (MANOVA), in which the three intention ratings serve as dependent measures. These analyses were performed for both the commissional and omissional intentions ratings. For the former, the main effect of direction was marginally significant $(p=.078)$, but no other effects were reliable. For the latter, only the 
None of the control group analyses of the Oll data revealed significant effects, and are therefore not described in detail.

Grade. Subjects' expected grade averages for the current academic year were subjected to a $2 \times 2 \times 2$ ANOVA. The main effect for direction was the only reliable effect in this analysis, $F(1,62)=7.49, p<.01$. The relation of the mean was the reverse of what was expected, however. Subjects considering upward alternatives expected a worse grade average $(M=72.2 \%)$ than those considering downward possibilities $(M=77.0 \%)$. The control group analysis shed some additional light on this surprising finding. The main effect from the omnibus oneway ANOVA in which all 5 conditions served as levels was significant, $F(4,80)$ $=2.84, p<.03$. The generation of upward alternatives had no impact on grade expectancies relative to the control group ( $M s=72.2 \%$ vs $72.3 \%$, respectively), $t(80)=.05, \mathrm{~ns}$. Consideration of downward counterfactuals, in contrast, resulted in heightened grade expectancies $(M=77.0 \%)$ relative to the control group, $t(80)$ $=2.28, p<.05$. The contrast between the four experimental conditions $(M=$ $74.6 \%)$ and the control condition was not significant, $\iota(80)=1.21$.

It is likely that these findings for grade expectancies are attributable to the influence of social desirability biases. The main effect for direction on grade expectancies was the only effect in this experiment to be moderated significantly by individual differences in social desirability. A 2 (direction) $\times 2$ (structure) $\times 2$ (SD) ANOVA performed on the grade estimates revealed the main effect of direction, $F(1,62)=10.55, p<.01$, but also an interaction between direction and $\mathrm{SD}, F(1.62)=10.97, p<.01$. It was clear from this analysis that direction had

structure by $\mathrm{SE}$ interaction was significant $(p<.01)$. 
no effect on grade estimates for LSD subjects $(M=74.9$ for upward; $M=74.7$ for downward). The effect occurred only for HSD subjects $(M=69.8$ for upward; $M=79.2$ for downward). Hence, the grade effect may be invalid, due perhaps to subjects' self-presentational concerns. The means for the intention ratings and grade expectancies are shown in Table 3.

\section{Attributional Ratings}

Unlike Experiment 1, the ratings of responsibility of self, responsibility of others, controllability, and avoidability yielded findings more consistent with the principal findings. ANOVAs performed on the ratings of the responsibility of others and of controllability did not reveal any reliable findings. However, a $2 \times 2$ $\times 2$ ANOVA applied to ratings of responsibility of self showed a significant main effect for direction, $F(1,62)=8.81, p<.01$. Subjects induced to consider better alternatives reported feeling more responsible for their poor grade $(M=8.06)$ than did those who considered worse alternatives $(M=6.89)$. The main effect from the omnibus oneway ANOVA in which all 5 conditions served as levels was marginally significant on this measure, $F(4,80)=2.37, p=.06$. A comparison of these means with the control condition indicated that this effect was due to the influence of upward counterfactual generation. These subjects reported greater responsibility than did those in the control condition $(M=6.93), t(80)=2.00, p<$ .05. whereas the difference between the means in the downward condition and the control condition was not reliable, $t<1$. The contrast between the four experimental conditions $(M=7.46)$ and the control condition was also not significant, $t<1$.

Similarly, another $2 \times 2 \times 2$ ANOVA showed that upward counterfactuals 
resulted in greater perceptions of the avoidability of receiving a poor grade ( $M=$ 6.77) than was the case following generation of downward counterfactuals $(M=$ 5.94), $F(1,62)=4.48, p<.05$. A significant main effect for $S E$ was also found, such that HSE persons saw their poor performance as more avoidable $(M=6.76)$ than LSE persons $(M=5.97), F(1,62)=4.27, p<.05$, perhaps reflecting the use of a defensive attributional strategy by HSE people. The omnibus oneway ANOVA was not significant for this measure $(F<1)$, and no reliable effects emerged in comparisons between these means and the control condition ( $M=$ 6.47), suggesting that the effect was due to the diverging influence of both upward and downward comparisons.

\section{Correlations}

Correlational analyses between direction-DV, structure-DV, the affect measures, and the expectancy ratings were also conducted. The first two variables are the same as those used in the parametric test of the manipulations. Table 4 displays the relevant correlations. Correlations between direction and affect, and also between direction and the OII and grade expectancies, support the conclusions from the ANOVAs. Siructure was unrelated to mood, whether the latter was operationalized using the mood scale or the disappointed-relieved measure. Direction was negatively related to the mood scale, $r(68)=-.25, p<$ .05 , such that more frequent consideration of upward counterfactuals covaried with more negative affective states. Similarly, direction was negatively related to the disappointed-relieved measure, $r(68)=-.24, p<.05$.

Table 5 displays the correlations between direction-DV, structure-DV, affect, and the four attributional ratings. This format permits a direct comparison 
Table 4

Correlations between counterfactual direction, structure, affect, and intention ratings

\begin{tabular}{|c|c|c|c|c|c|c|}
\hline & ST & MS & DR & CII & OlI & GR \\
\hline Direction-DV & $.22^{*}$ & $-.25^{*}$ & $-.24^{*}$ & .14 & $.25^{*}$ & $-.29^{* *}$ \\
\hline Structure-DV & & .05 & .07 & -.09 & .03 & -.12 \\
\hline Mood Scale & & & $.73^{* *}$ & .01 & .14 & -.09 \\
\hline \multicolumn{3}{|c|}{ Disappointed/Relieved } & & .01 & -.07 & -.09 \\
\hline \multicolumn{3}{|l|}{ CII } & & & $.33^{* *}$ & . 199 \\
\hline \multicolumn{3}{|l|}{ OII } & & & & .08 \\
\hline
\end{tabular}

Note. For Direction-DV, higher numbers indicate more frequent upward counterfactuals; ST $=$ Structure-DV (higher numbers indicate more frequent additive counterfactuals); MS = mood scale (for both affective measures, higher numbers indicate more positive affect); $\mathrm{DR}=$ disappointed-relieved rating: $\mathrm{CII}=$ commissional intention index; $\mathrm{OII}=$ omissional intention index; $\mathrm{GR}=$ expected grade average. $N=70$ (control subjects were not included in this analysis). ${ }^{*} p<.05 ;{ }^{* *} p<.01$. 
Table 5

Correlations between counterfactual direction, structure, affect, and attributional ratings

\begin{tabular}{|c|c|c|c|c|c|c|c|}
\hline & ST & MS & DR & $\mathbf{R} 1$ & $\mathbf{R 2}$ & R3 & $\mathbf{R 4}$ \\
\hline Direction-DV & $.22^{*}$ & $-.25^{*}$ & $-.24^{*}$ & $.28^{* *}$ & .09 & .02 & .12 \\
\hline Structure-DV & & .05 & .07 &. .10 & .07 & .02 &. .04 \\
\hline Mood Scale & & & $.73^{* *}$ & .00 & .09 & -.02 & -.11 \\
\hline \multicolumn{2}{|c|}{ Disappointed/Relieved } & & & -.11 & .15 & -.03 & -.02 \\
\hline R1 & & & & & $.29 * *$ & $.30^{* *}$ & $.33^{* *}$ \\
\hline $\mathbf{R} 2$ & & & & & & $-.25^{*}$ & $-.35 * *$ \\
\hline $\mathbf{R 3}$ & & & & & & & $.44^{* *}$ \\
\hline
\end{tabular}

Note. For Direction-DV, higher numbers indicate more frequent upward counterfactuals; ST = Structure-DV (higher numbers indicate more frequent additive counterfactuals); DR = disappointed-relieved affect measure; $\mathrm{MS}=$ mood scale (for both affective measures, higher numbers indicate more positive affect); $R 1$ = rating of personal responsibility; $R 2$ = rating of responsibility of others; $\mathbf{R 3}=$ rating of outcome controllability; $\mathbf{R 4}=$ rating of cutcome avoidability (for all attributional ratings, higher numbers indicate more of the specified quality). $N=70$ (control subjects were not included in this analysis). ${ }^{*} p<.05 ;{ }^{* *} p<.01$. 
with the same correlations presented in Experiment 1 (see Table 2). Unlike Experiment 1, perceptions of avoidability were unrelated to affect. The positive relation between direction-DV and perceptions of personal responsibility reflects the ANOVA finding previously mentioned (consideration of upward counterfactuals tends to make people feel more responsible for a personal failure). No other correlations were informative.

\section{Discussion}

The main objectives for Experiment 2 were, for the most part. met. First, the manipulations of counterfactual direction and structure proved effective.

Second, reliable effects of counterfactual direction were found on both the gishal and specific affect measures. Third, reliable effects of counterfactual direction were noted for the intention measures.

The concerns raised by the $2 \times 2$ manipulation of direction and structure in Experiment 1 were addressed and assuaged. The manipulation of these two variables in the present experiment resulted in the appropriate, predicted effects on both the nonparametric and parametric manipulation checks. Thus. it indeed appears feasible to manipulate these variables experimentally. The manipulation check revealed one zotentially interesting finding: HSE persons may 'end to generate upward counterfactuals more easily when instructed to do "o than LSE persons. This may reflect the fact that HSE persons are better able to make use of the preparative effects of upward counterfactuals; this more numerous generation did not, however, re:ult in any negative affective consequences for these people relative to LSE people. 
Counterfactual direction was found to have a significant impact on affect. As predicted hut unlike Experiment 1, there was a significant main effect for direction on the glos al measure of affect (i.e., the mood scale). Downward count - rfactual generation resulted in more positive feelings than did upward counterfactual generation. Thus, the failure to obtain this effect in the first experiment was likely due, as argued, to the problems with the manipulation and the sample size rather than to problems with the conceptual basis for the predictions. Moreover. the employment of a contro! grour permitted a more specific description of this effert. Relative to o-counterfactual control subjects, down ward subjects reported more positive affect. but ur,ward subjects reported no more negative aifect. The pa:tern of means of the more specific disappointedrelieved rating paralleled this finding exactly.

These results both support and extend the findings of Markman et al. (1993). These authors found that two experimental manipuldtions that: increased the generation of downward as opposed to upward counterfactual thoughts also increased subjects' reported levels of satisfaction with a given outcome. Even after partialling out the contribution of the tivo manipulations, direction and satısfaction were still found to be negatively correlated. The present results piovide a more direct demonstration of the causil impact of direction on structure. A manipukation designed explicitly to produce either upward or downward countertactuals shifted affect in the same manner as show by Markman et al. as assessed by hoth a glohal and a specific mood measure. The use of the more global measure of affect extends the Markmal 't al. findings synificant!y. the emotional consey!ences of counterfactual direction are not limite: to domars-specific (or conceptually tautological) emotional reactions. but 
can apparently extend to more general mood states. The inclusion of a nocounterfactual control group also provides an important extension of the Markman et al. findings. Their results were ambiguous regarding the exact nature of affect differences as a function of upward versus downward counterfactuals. The present results showed that the main effect of direction may not be a result of the affect-depressing effect of upward counterfactuals (i.e., the mean for this group did not differ reliably from the control group mean) but rather of the positive affect-enhancing effect of downward counterfactuals. The implications of these findings are discussed more fully in the General Discussion.

As predicted, consideration of upward counterfactuals led to increased intentions to perform success-facilitating behaviours. These behaviours were described as commissional, reflecting their active nature (e.g.. going to visit professors for extra help). The inclusion of a control group again permitted a closer examination of this efiect. Upward counterfactuals were found to be largely responsible for : te main effect: subjects in this condition reported significantly greater intentions than no-counterfactual control subjects. Subjects generating downward counterfactuals did not differ reliably in their intentions from those in the control group. This finding confirms a significant portion of the hypothesized mechanism underlying the preparative function (i.e., linking counterfactuals to behaviour). Manipulations of counterfactual thoughts can indeed have an effect on behavioural intentions.

Contrary to expectations, the effects of counterfactual structure were not reliable. It was predicted that additive counterfactuals would result in heightened commissional intentions relative to subtractive counterfactuals. Predictions regarding the impact of structure on omissional intentions were somewhat less 
clear, however. There is at least one possible explanation for these null findings, beyond the simplest one that structure does not serve a preparative function. It may be that additive counterfactuals exert their preparative effect in a more highly circumscribed, domain-specific manner than upward countertactuals. Thus, although upward counterfactuals may induce broader (and perhaps more motivationally-based) effects on a variety of intentions, the effects of additive counterfactuals might be more specific, both conceptually and temporally. If this is the case, then a retrospective self-report design. coupled with vague future possibilities, would not represent the most appropriate test of the effects of additive versus subtractive counterfactuals. In Experiment 3, subjects generated counterfactuals in response to a narrowly circumscribed task for which more specific facilitative actions were possible, and that could be employed almost immediately. This third experiment, according to this logic. provides a more sensitive test of the effects of counterfactuai structure.

Omissional behaviours were defined in terms of not performing an action inhibitory of success (e.g. not watching TV before an exam). The results using this variable as a dependent measure were somewhat less clear. The predicted main effect of counterfactual direction was only marginally significant, with the means falling in the predicted direction. Ar interaction between structure and SE seemed to indicate that only HSE persons are sensitive to the structure Ihanipulation. For these subjects, additive counterfactuals increased their intentions to refrain from performing success-inhibiting hehaviour.. However. the possibility that this dependent measure was tainted by social desirability blases limits the strength of these conclusions.

The effect of direction on grade expectancies appeared to be contrary to 
predictions. However, as noted in the results section, this measure was likely tainted by social desirability biases, thus bringing into doubt the validity of any findings obtained from it. The grade expectancy findings are therefore not discussed further.

In sum, the second experiment provides important evidence that counterfactuals can serve an affective function. The direction of comparisons is clearly important for understanding this function: downward counterfactuals caused subjects to report more positive affect; however, upward counterfactuals did not engender more negative affect relative to controls. The experiment also provides evidence that counterfactuals can serve a preparative function. Upward counterfactuals increased intentions to perform success-facilitating behaviours. Unlike the affective function, this effect seemed to be due mainly to the effects of upward counterfactuals; downward counterfactuals did not engender lower intentions relative to controls. 


\section{CHAPTER IV}

\section{EXPERIMENT 3}


The second experiment provided important evidence in support of the proposed mechanism underlying the preparative function. That is, counterfactuals were shown to be causally related to behavioural intentions. The third experiment was designed to complement this finding by providing evidence for the causal linkage between counterfactuals and behaviour. Subjects completed a computeradministered anagram task twice. Counterfactuals were generated between the two task performances. Once again, both direction and structure were manipulated, and a fifth no-counterfactual control condition was included. Improvement in performance from the first to the second task represented a behavioural index of preparative counterfactual effects. Consistent with Experiment 2, counterfactual direction was predicted to influence performance, such that upward counterfactuals would enhance performance to a greater extent than downward counterfactuals.

In the discussion of Experiment 2, it was suggested that the failure to detect an effect of counterfactual structure may have been due to its more domain-specific nature. If that is the case, then this third experiment would represent a more sensitive test of its effects. That is, if adritive as opposed to subtractive counterfactuals represent an efficient means of illuminating facilitative acts that are highly domain specific, then a more appropriate test would be one in which the dependent measures are linked to behaviours performed soon after counterfactual generation. Thus, a main effect of counterfactual structure was predicted on the performance measure, such that additive as opposed to 
subtractive counterfactual generation would result in enhanced performance.

The affective function assumed a more secondary role in this experiment. Affect was assessed directly following counterfactual generation, but since no procedural or design modifications were effected, this test represents a simple replication of the findings of Experiment 2. Consideration of upward counterfactuals was expected to result in more negative affect than consideration of downward counterfactuals. This effect was expected on both the global affect measure as well as the disappointed-relieved measure. Self-esteem was also assessed, but owing to the failure to find any moderating impact of this individual difference variable on the affective findings in the previous experiments, no specific effects for SE were predicted.

It is important to note that previous ccunterfactual experiments have relied almost exclusively on simple scenario methodologies (the Markman et al., 1993 study is the only published exception). Experiments 1 and 2 extended this methodological domain, at least somewhat, through the use of retrospective selfreports. Both of these methods, although informative, suffer from a lack of involvingness, as well as from the possibility of self-report biases. With the use of a more involving skills-based task, the present experiment extends the methodological range of the extant counterfactual literature. Not only is the task involving and challenging, but the use of a behavioural dependent measure precludes interpretational ambiguity from self-report biases. 


\section{Method}

\section{Subjects}

Participants were 83 (48 women, 35 men) introductory psychology studerits attending the University of Western Ontario, randomly assigned to the five experimental conditions, with the constraint of roughly equal cell sizes. All participants received course credit in exchange for participation. Three subjects were eliminated from the original sample of 86 because of failure to complete fully all experimental materials.

\section{Materials and Procedure}

Cverview. Subjects completed two sets of 10 anagrams presented on computer. After completing the first set, all subjects received failure feedback, then generated counterfactual alternatives to their performance. A fifth control group underwent the same procedure, with the exception that these subjects generated no counierfactuals. Subjects rated their mood, rated their expected performance on the second anagram task, then completed a short filler task. They then completed a second set of 10 anagrams, and finally, completed individual difference measures.

Upon entering the laboratory, subjects were seated at a large central table. and briefly informed that they were participating in a study examining "decisionmaking under time pressure." Subjects signed consent forms, then were seated in individual rooms for the duration of the experiment. Up to four subjects were run ai one time.

Located on a table in each room was an IBM-compatible microcomputer. 
The anagram-solving protocol was directed by a BASIC-language program preloaded into the computers. The complete computer program is included in Appendix E. Subjects pressed a key to begin, and the following instructions appeared onscreen:

This is a study of decision-making processes under time pressure. Your task will be to solve a series of anagrams within a certain period of time. Anagrams are scrambled woid combinations -- solving them simply means unscrambling them into an actual word. For example, 'YHAPP' is an anagram and its solution is 'HAPPY'. All the anagrams in this study have only ONE solution.

For each anagram solved, you will be awarded a certain number of points. At the end of the anagram task, the computer will calculate a total score, and then it will tell you how good this score is compared to other participants in this study.

You will be awarded a maximum of 120 points for each anagram solved. However, the longer it takes for you to solve the anagram, the lower your score. One point will be deducted per second. So if you took 20 seconds to solve an anagram, you would get 100 points; if you took 60) seconds to solve it, you would get only 60 points. You have a total of 2 minutes (120 seconds) to solve each anagram. After 2 minutes have elapsed, the computer will give you the solution and present :he next anagram 
But that's not all. If you cannot solve the anagram within the 2 minute period, you will lose points. One point will be deducted from your TOTAL score for each second spent on an anagram you didn't solve. This means that it's a good idea to SKIP OVER anagrams that seem especially difficult; this allows you to minimize the points lost. Therefore, deciding which anagrams to skip c:er is one of the most important decisions you'll make in this task. You can skip ahead at any time simply by pressing $\langle\mathrm{S}\rangle$ on your keyboard.

If you think you are near to solving the anagram but can't quite get it, you may 'buy' a clue. This will cost you 30 points from your score. The clue will be the middle letter of the correct solution. So for the anagram 'YHAPP', you would get the clue 'P', which is the middle letter of the solution 'HAPPY'. You can i zquest this clue at any time by simply pressing $<\mathrm{C}>$ on your keyboard.

When you figure out the solution to the anagram, press $<R>$ on your keyboard, then type the five letters in, then press $<$ ENTER $>$. The computer will tell you immediately if you are right or wrong. Don't worry about remembering which letters to press. The computer will remind you which ones you need to know.

One last thing. A timer will be displayed onscreen to let you know how much time you have left. When you type in your solution, the timer display shuts off. However, YOUR TIME IS STILL RUNNING OUT! The clock 
inside the computer will stop ONLY when a correct solution has been entered. So it's a good idea to type in your solution (and then press the <ENTER > key) as FAST AS YOU CAN.

If you need to read over these instructions a second time, press $\langle G\rangle$. If anything is unclear to you, please ask the experimenter now. Otherwise, press $<\mathrm{C}>$ to continue.

Subject then had the opportunity to decide upon several parameters associated with the task. First, subjects chose the intertrial interval (i.e., the delay between successive anagram presentations). This choice was restricted to between 1 and 9 seconds. Second, subjects chose the topic area from which the anagrams were se'ected (either "people and events" or "science and nature"). Third, subjects chose the difficulty level of the anagrams (either "easy" or "difficult"). The difficult anagram set purportedly involved 10 bonus points for each anagram solved. In actuality, this was a dummy choice; all subjects were assigned relatively difficult anagrams and no bonus points were awarded.

The anagram task then commenced. Subjects faced a multi-coloured video display with the anagram in the central field, a small digital clock in the lower right-hand corner (counting down in minutes, seconds, and milliseconds), and the ke,-press options listed along the right edge of the screen. As noted in the computer instructions, subjects could skip over any anagram at any time and they could select clues at any time. This degree of "control" over the experimental task was important for two reasons. First, these decisions represented obvious mutation targets; that is, antecedent elements out of which subjects could create 
counterfactual alternatives to their performance. Second, it permitted subjects to formulate strategies that could indeed facilitate performance.

The anagrams themselves were selected on the basis of previous cognitive research. Specifically, the anagrams were selected from a study published by Gilhooly and Johnson (1978), in which a pool of 80 5-letter anagrams were used to assess the predictors of solution time. These anagrams had no repeated letters, were not plurals, could be used as nouns, and had only one correct solution. Four sets of 10 anagrams were created from this pool. Two focused (although somewhat loosely) on the topic of "people and events", whereas the other two focused on "science and nature". These four sets are disflayed in Table 6. The first two sets given in this Table represented the choice subjects could make for the first anagram task (i.e., between the topics): the second two sets formed the basis for the topic choice of the second task. All four sets were equated on the dimensions identified by Gilhooly and Johnson as determinants of anagram solution time (bigram rank, pronounceability, anagram-word similarity, vowel vs consonant starting letter). Thus, differences in solution times across sets (either as a function of subjects' topic choice or as a function of the first vs the second anagram task) could not be attributed to differences in anagram difficulty of the respective sets.

:ollowing completion of the first anagram set, all subjects received accurate information regarding their total score. Subjects also learned the number of anagrams correctly solved, the number of skips. and the number of clues used. H.wever, all subjects received failure feedback. This was achieved by presenting subjects with false information regarding the normative performance of all subjects run through the study to that point. Subjects were shown a number purportedly 
Table 6

Anagrams (with solutions) used in Experiment 3

1. gbtio (bigot), owamn (woman), ordcw (crowd), fteih (thief), gifth (fight), ftlri (flirt), fiyra (fairy), criav (vicar), cnhlu (unch), trhim (mirth)

2. ioglc (logic), githl (light), srivu (virus), hnduo (hound), itruf (fruit), iptlu (tulip), ofrec (force), noehy (honey), retiv (rivet), yaorv (ovary)

3. ownlc (clown), augrd (guard), eunde (dunce), zltwa (waltz), oustc (scout), hslac (clash), ongya (agony), kasnc (snack), emycr (mercy), bnhuc (bunch)

4. botir (orbit), hsatl (flash), htiwd (width), ijnot (joint), haeny (hyena), phted (depth), hecpr (perch), tcuon (count), plimb (blimp), chitp (pitch)

Note. Subjects chose between anagram sets 1 and 2 for the first anagram task. and between sets 3 and 4 for the second task. Sets 1 and 3 were labelled "people and events", and sets 2 and 4 were labelled "science and nature". 
indicating the average score of all subjects. In actuality, this value was their own score plus 53, calculated automatically hy the computer program. Directly helow this, another statement indicated to subjects that their rank out of all participants was 37 th out of 45 .

At this point, the computer program paused, and could not be reactivated unless a password were eritered. An onscreen message indicated that subjects should open their door $t_{1}$ signal to the experimenter that they were ready for the next task. The experimenter provided subjects with a questionnaire borklet containing mood and expectancy dependent measures, as well as a filler task.

The first page of the booklet contained the counterfactual manipulation. presented in a marner similar to Experiments 1 and 2. Counterfactual direction and structure were varied orthogonally in a $2 \times 2$ factorial design. Upward combined with additive counterfactuals were solicited as follows:

People often evaluate their past performance with thoughts like "if only ... ". These thoughts focus on how things might have turned out better. For example, a Toronto woman who recently sustained minor injuries when she was hit by a car told reporters, "If only I had looked down that street a second time, I wouldve been fine." Often. we wish we had done something to avoid a certain outcome. In the space below, please list any speciacic actions that you could have taken to improve your anagram scors.

Upward combined with subtractive counterfactuals were requested in the following manner: 
People often evaluate thsir past performance with thoughts like "if only ... ". These thoughts focus on how things might have turneci out better. For example, a Toronto woman who recently sustained minor injuries when she was hit by a car told reporters, "If only I had NOT been in such a rush, I would've been fine." Often, we wish we hadn't done something that led to a certain outcome. In the space below, please list any specific actions that you should not have taken to improve your anagram score.

Downward combined with additive counterfactuals were requested in the following manner:

People often evaluate their past performance with thoughts like "well, at least ... ". These thoughts focus on how things might have turned out even worse. For example, a Toronto woman who recently sustained minor injuries when she was hit by a car told reporters, "At least I didn't try to move around after the accident, or it would've been a lot worse." Often, there are things that could have happened that would have made a given outcome worse. In the space below, please list any specific artions that you could have taken to make your anagram score worse.

Downward combined with subtractive counterfactuals were requested in the following manner:

People often evaluate their past performance with thoughts like "well, at least ... ". These thoughts focus on how things might have turned out even 
worse. For example, a Toronto woman who recently sustained minor injuries when she was hit by car told reporters, "At least I had my medicalert bracelet on, or it would've been a lot worse." Often, negative outcomes could have been worse if certain actions had not been taken. In the space below, please list any specific actions that you believe enhanced your anagram score, and that would make your anagram score worse if you hadn't performed them.

These written solicitatic 'ns were accompanied by an oral admonishment to record only the counterfactual subtype specified. Subjects could record as many counterfactual thoughts as they desired (an entire page was left blank for this purpose). A fifth, control condition was also used. Subjects in this condition completed tasks identical to the other four conditions, with the exception that they generated no counterfactual thoughts. Because the oral admonishment did not occur until after subjects were seated in individual rooms, control subjects could be run at the same time as the four experimental conditions.

Subjects next made affective ratings in response to the request, "please indicate your reaction to your anagram score by circling a number on each of the following scales." Eight bipolar items appeared in the following order: disappointed-relieved, concerned-unconcerned, depressed-elated, tired-awake, negative-positive, anxious-calm, unhappy-happy, hostile-agreeable. All ratings were made on 9-point scales ranging from -4 to +4 . These ratings were identical to those used in Experiment 2. The same 5 items as in Experiments 1 and 2 were summed to yield a composite mood scale.

Subjects then made the following 9-point ratings on scales ranging from -4 
to +4: 1) "How responsible were you for your anagram score?" (scale anchored by "not at all responsible" and "completely responsible"), 2) "How difficult did your anagrams seem to you?" (scale anchored by "not at all difficult" and "extremely difficult"), 3) "How much control over your anagram score did you have?" (scale anchored by "not at all under my control" and "completely under my control"), and 4) "How avoidable was your anagram score?" (scale anchored by "not at all avoidable" and "completely avoidable").

On the last page of the booklet, subjects were informed for the first time that they would attempt to solve a second set of anagrams. This was an important feature of the procedure: it ensured that the counterfactuals considered by subjects focused explicitly on a past outcome. At this point, subjects were asked, "How do you expect to perform on this next set of anagrams?". A 5-point response scale ranging from -2 to +2 was labelled with "much worse", "a little bit worse", "about the same", "a little bit better", and "much better". Subjects then completed another booklet containing filler materials irrelevant to the present experiment (this filler task consisted of trait ratings made for subjects' acquaintances, and took about 5 minutes to complete).

When both booklets had been completed, subjects were given the password that would reactivate the computer program. The second anagram set followed the exact same format as the first, with another series of 10 anagrams presented sequentially by computer. Following the completion of this second set, subjects were again presented with accurate information regarding their total score, the number of anagrams correctly solved, the number of skips, and the number of clues used. No false information regarding the normative performance of other subjects was provided. 
The last phase of the procedure involved the completion of the TSBI and the SD scales. The format of the administration of these scales was identical to that of Experiments 1 and 2.

At the completion of each experimental session, subjects were fully debriefed and provided with a written description of this research and its goals. These and all other written materials used in this experiment are included in Appendix D. Because of the necessity of experimental use of false negative feedback, extra care was taken to explain the details of the deception. The nature of belief perseverance was briefly described, and subjects were told that, because of the difficulty of the anagrams, they had done quite well if they solved at least two anagrams per set (the average was $>5$ correct per set, and no subject solved fewer than two on both sets).

\section{Results}

Individual Difference Measures

Scores on the TSBI self-esteem measure ranged from 56 to $133(M=93.8$; $M d=95$ ). This scale achieved adequate internal reliability (Cronbach's $\alpha=.82$ ). A median split was performed on the SE scores to produce HSE and LSE groups. Scores on the social desirability measure ranged from 14 to $65(M=46.5 ; M d=$ 47). This measure failed, however, to achieve a satisfactory level of internal reliability $(\alpha=.43)$. The unreliability of this scale precluded its use in subsequent analyses; hence, it is not discussed further.

Sex of subject was not significant as a main effect or as part of interaction effects, and thus is not discussed further. 


\section{Manipulation Checks}

The nonparametric manipulation check was, as in the previous experiments, based on a dichotomous classification of whether (Ir not subjects correctly followed instructions, recording the appropriate counterfactual type corresponding to their experimental conditions." Subjects were classified as "correct" if the number of counterfactuals corresponding to their condition exceeded all other noncorrespondent counterfactuals. All but 8 of 83 correctly followed instructions, thus yielding a $90 \%$ success rate for the manipulation. Of these 8 , one was in the upward/additive condition, 3 were in the upward/subtractive condition, one was in the downward/additive condition, and 3 were in the downward/subtractive condition. The 8 exceptions were eliminated from all subsequent analyses, thus yielding a final sample of $75 .^{13}$

The parametric manipulation check was based on the same two variables defined in Experiments 1 and 2, direction-DV and structure-DV. A 2 (direction) $\times 2$ (structure) $\times 2$ (SE) ANOVA, using direction-DV as the dependent measure, revealed the expected main effect for direction, $F(1,52)=225.09, p<.001$. Upward counterfactuals were more frequent in the upward condition and downward counterfactuals were more frequent in the downward condition $(M \mathrm{~s}=$ $2.87,-2.63$, respectively). The manipulation of structure had no effect on

12 As with the other two experiments, a second rater coded both direction and structure on a random sample of 24 subjects' responses (32\% of the sample). The inter-rater agreement was again excellent (100\% for direction, $98 \%$ for structure). Examples of each subtype appear in Appendix A.

${ }^{13}$ Analyses conducted on the full sample of 83 yielded the same conclusions presented in the text. 
direction-DV $(F=.08, \mathrm{~ns})$, and no other effects were reliable in this analysis.

Another $2 \times 2 \times 2$ ANOVA performed on structure-DV revealed the predicted main effect of structure, $F(1,52)=153.63, p<.001$, with the means falling in the expected directions $(M=2.90$ in the additive ondition; $M=-2.13$ in the subtractive condition). As expected, the direction manipulation had no effect on this measure $(F=.07, \mathrm{~ns})$. No other effects were reliable in this analysis. The manipulations of counterfactual direction and structure were, therefore, again implemented successfully.

\section{Affect}

Mood wale. A 2 (direction) $\times 2$ (structure) $\times 2$ (SE) ANOVA was performed on the mood scale (comprising the 5 mood items). This scale was reliable $(\alpha=.80)$. None of the main effects from this analysis was significant. There was, however, a significant 3 -way interaction, $F(1,52)=5.05, p<.05$. The means for this interaction are displayed in Table 7. Pairwise contrasts between LSE and HSE persons within levels of direction and structure were used to elucidate this interaction. Only the SE contrast within the upward/subtractive condition was significant, $t(52)=2.81, p<.05$. LSE persons reported more positive affect $(M=5.45)$ than HSE persons $(M=3.83)$ only in the upward/subtractive condition. This finding is not readily interpretable.

Disappointed-relieved. Another $2 \times 2 \times 2$ ANOVA was used to examine the single disappointed-relieved rating. There were no significant effects in this analysis. The cell means for both affect measures as a function of the two manipulated variables are presented in Table 8. 


\section{Table 7}

Interaction effect between direction, structure, and SE on affect ratings

Condition

(Direction/Structure)

Upward/Additive

Upward/Subtractive

Downward/Additive

Downward/Subtractive
Self-Esteem

LSE HSE

4.33

4.89

5.45

3.83*

4.77

4.58

4.40

4.64

Note. Values represent mean mood scale ratings; higher numvers signify more positive affect. ${ }^{*}$ SE difference $\rho<.05$. 
Table 8

Summary of within-cell means of the principal dependent measures

\begin{tabular}{lccccc}
\hline & \multicolumn{5}{c}{ Condition } \\
\cline { 2 - 6 } Measure & $\begin{array}{l}\text { Upward/ } \\
\text { Additive }\end{array}$ & $\begin{array}{l}\text { Upward/ } \\
\text { Subtractive }\end{array}$ & $\begin{array}{l}\text { Downward/ } \\
\text { Additive }\end{array}$ & $\begin{array}{l}\text { Downward/ } \\
\text { Subtractive }\end{array}$ & Control \\
\hline Mood Scale & 4.59 & 4.69 & 4.65 & 4.55 & 5.20 \\
Dis/Rel & 3.07 & 2.47 & 2.80 & 2.93 & 4.00 \\
$n$ & 15 & 15 & 15 & 15 & 15 \\
\hline
\end{tabular}

Note. Dis/Rel $=$ disappointed-relieved rating (for both affective measures, higher numbers indicate more positive affect); $n=$ number of subjects per cell. 


\section{Expectancy}

A single 5-point rating was used to assess subjects' expectancy for their performance on the second anagram task. A $2 \times 2 \times 2$ ANOVA used to assess this dependent measure yielded no reliable effects. This was likely due to a ceiling effect: most subjects expected to perform better on the second task ( $M:=$ 3.92). This ceiling effect was perhaps compounded by the fact that the scale was based on only 5 points. The majority of subjects chose options $4(58.3 \%)$ or 5 $(16.7 \%)$, resulting in little variance, $S D=.68$.

\section{Performance}

Total score. As described in the Method section, the total score on the anagram task was a function of the total number of anagrams solved weighted by the time it took to solve them, with the number of anagrams not solved weighted by time spent on them subtracted from this value. The average score on the first anagrarn set was 296.8; the average on the second was 472.0 . Thus, the average improvement from set one to set two was 174.2. For each subject, an improvement score was calculated by subtracting their score on set one from their score en set two. These improvement scores were then submitted to a $2 \times 2 \times 2$ ANOVA. ${ }^{14}$ All three main effects were reliable. As predicted, subjects in the upward counterfactual condition improved more $(M=232.0)$ than those in the

14 A repeated measures ANOVA in which scores on the first and second anagram tasks serve as levels of a within-subject variable would represent a more statistically appropriate means of testing these effects (e.g., Cronbach \& Furby, 1970). Such an ANOVA was conducted, and yielded exactly the same conclusions as the difference score ANOVA presented in the text. The latter, however, has the advantage of greater clarity and ease of presentation, and thus is used exclusively. 
downward condition $(M=118.3), F(1,52)=4.72, p<.05$. Also as predicted, subjects in the additive condition improved more $(M=284.4)$ than those in the subtractive condition $(M=65.8), F(1,52)=12.65, p<.01$. HSE subjects improved more $(M=240.3)$ than LSE subjects $(M=100.7), F(1,52)=6.43, p<$ .02. See Figures 4 and 5 for depictions of the main effects of the two manipulated variables. Cell means within levels of the manipulated variables are presented in Table 9 (higher scores in this figure indicate greater improvement).

The analysis also revealed a 3-way interaction, $F(1,52)=5.72, p<.05$, depicted in Table 10. Pairwise contrasts between LSE and HSE persons within levels of direction and structure were used to elucidate this interaction. The only contrast to achieve significance was within the upward/subtractive condition. HSE persons improved more $(M=380.7)$ than LSE persons $(M=-15.3)$ following the generation of upward/subtractive counterfactuals, $t(52)=3.21, p<.01$. This finding suggests that HSE persons may be better able to take advantage of subtractive counterfactuals that focus on better alternatives than are LSE persons.

Control group analyses were employed in the same manner as in Experiment 2. Thus, contrasts were used that were based on the mean square error from an omnibus oneway ANOVA in which all 5 experimental conditions served as levels. The main effect was significant from this analysis, $F(4,70)=$ 4.14, $p<.01$. Improvement scores in the upward counterfactual condition $(M=$ 232.0) did not differ reliably from the scores in the control condition $(M=119.3)$, $1(70)=1.40$, ns. Improvement scores in the downward counterfactual condition $(M=118.3)$ were almost identical to those in the control condition, $t<1$. Comparisons involving the counterfactual structure dimension proved more interesting. Subjects in the additive condition $(M=284.4)$ improved more than 
Figure 4

Effect of counterfactual direction on improvement scores

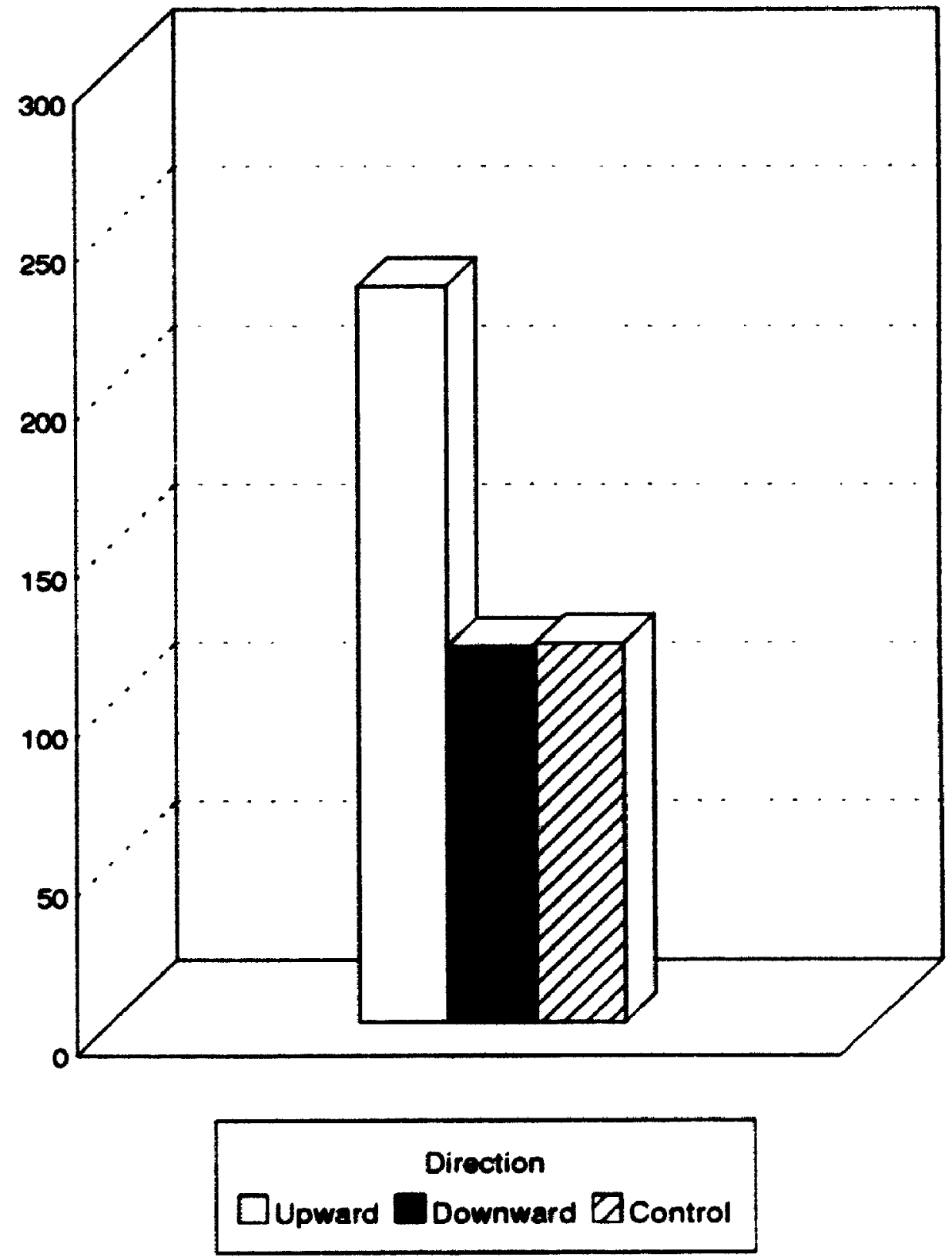


Figure 5

Effect of counterfactual structure on improvement scores

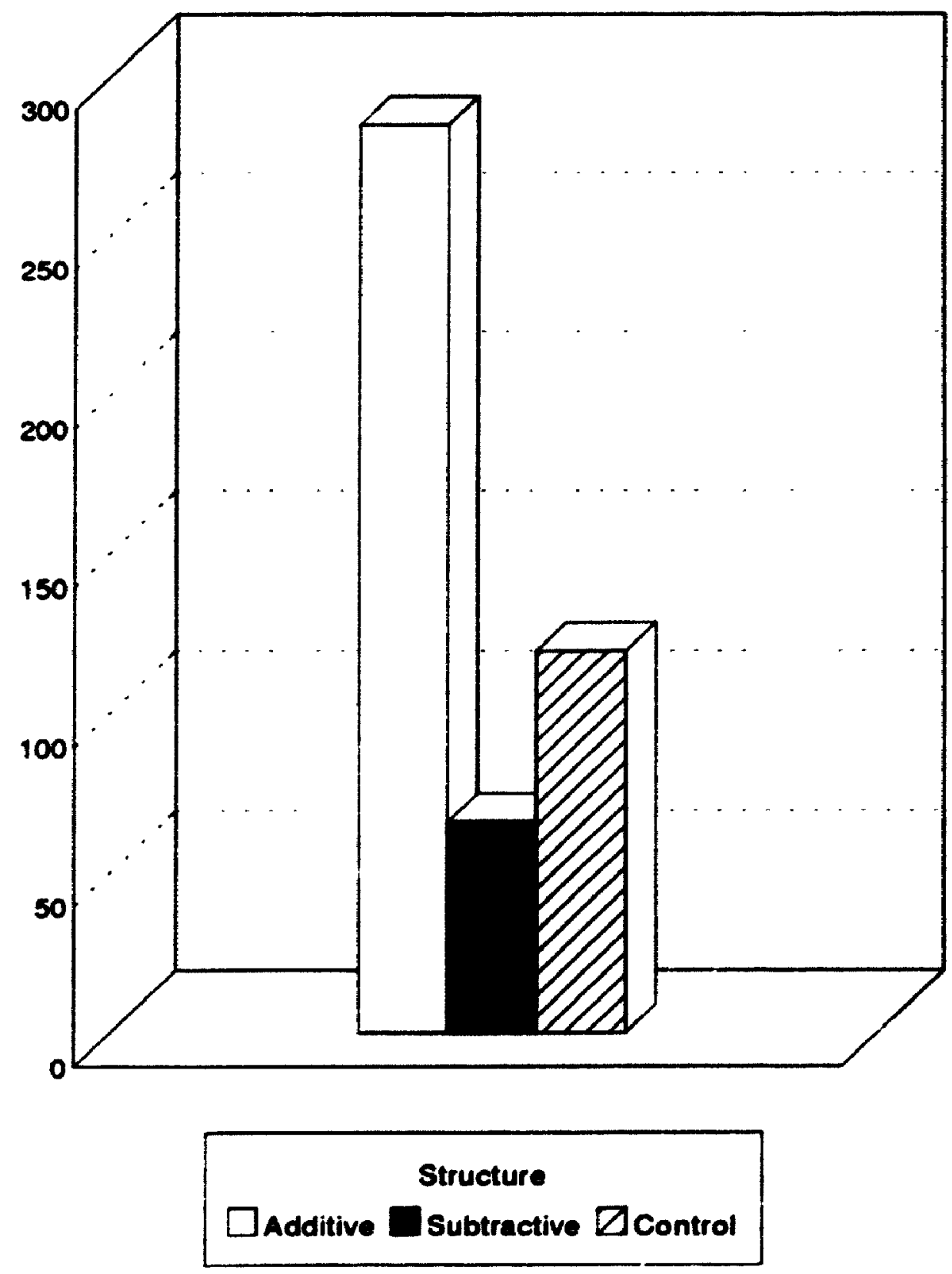


Table 9

Summary of within-cell means of the principal dependent measures

\begin{tabular}{lccccc}
\hline & \multicolumn{5}{c}{ Condition } \\
\cline { 2 - 6 } Measure & $\begin{array}{l}\text { Upward/ } \\
\text { Additive }\end{array}$ & $\begin{array}{l}\text { Upward/ } \\
\text { Subtractive }\end{array}$ & $\begin{array}{l}\text { Downward/ } \\
\text { Additive }\end{array}$ & $\begin{array}{l}\text { Downward/ } \\
\text { Subtractive }\end{array}$ & Control \\
\hline SC1 & 227.3 & 343.5 & 218.1 & 398.4 & 347.8 \\
SC2 & 521.7 & 513.1 & 492.5 & 360.5 & 467.1 \\
SCI & 294.4 & 169.6 & 274.5 & -37.9 & 119.3 \\
C1 & 4.67 & 5.60 & 4.40 & 5.80 & 6.13 \\
C2 & 6.67 & 6.80 & 6.33 & 6.27 & 6.73 \\
CI & 2.00 & 1.20 & 1.93 & .47 & .60 \\
$n$ & 15 & 15 & 15 & 15 & 15 \\
\hline
\end{tabular}

Note. $\mathrm{SC} 1=$ total score on first anagram set; $\mathrm{SC} 2=$ total score on second anagram set; $\mathrm{SCI}=$ improvement in performance, calculated by subtracting $\mathrm{SCl}$ from $\mathrm{SC2} ; \mathrm{C1}=$ number of anagrams correctly solved on first anagram set: $\mathrm{C2}=$ number of anagrams correctly solved on second anagram set; $\mathrm{Cl}=$ improvement in number of anagrams correctly solved, calculated by subtracing C1 from C2: $n$ = number of subjects per cell; UA = upward/additive condition; US = upward/subtractive condition; DA = downward/additive condition; DS = downward/subtractive condition. 
Table 10

Interaction effect between direction, structure, and SE on performance

Condition

(Direction/Structure)

Upward/Additive

Upward/Subtractive

Downward/Additive

Downward/Subtractive
Self-Esteem

LSE HSE

324.1

$-15.3$

144.7

$-86.7$
260.4

$380.9^{*}$

361.0

$-5.4$

Note. Values represent improvement in performance, calculated by subtracting the total score on the first anagram set from the total score on the second set. Thus, higher numbers indicate greater improvement. * SE difference $p<.05$. 
those in the control condition, $l(70)=2.04, p<.05$. However, those in the subtractive condition $(M=65.5)$ did not differ from those in the control condition, $t<1$. Thus, the main effect of structure on improvement is due largely to the performance-enhancing effect of additive counterfactuals. A contrast in which the means of the four experimental conditions were compared to that of the control condition was not significant, $t<1$.

Number correct. The number of anagrams correctly solved could also be used as a dependent measure. Indeed, the within-cell means for the first and second task are shown in Table 9. Analyses of this measure would be statistically inappropriate since this measure overlaps considerably with the principal performance measure (the two differ only in the weighting by solution time of the latter). The correlation between the two measures was $r(58)=.88, p<.001$. Nevertheless, the main findings presented above are replicated when this measure is analysed, with the exception that the main effect of direction is not significant. However, this measure is clearly less sensitive to the unique demands of the anagram task, since subjects were acting on the belief that solution time was an important component of their achievement.

Clues and Skips. Subjects' use of clues and skips (which were saved automatically by the computer program) represent specific behaviours that could potentially improve their performance. Indeed, across total scores for both sets, those who chose fewer clues performed better (average within-cell $r[73]=-.49, p$ $<.001$ ), and those who skipped over anagrams less frequently also performed better (average within-cell $r[73]=-.73, p<.001$ ). Of course, the causal direction of these correlations is ambiguous: Subjects may have performed better because they deliberately selected fewer clues and made fewer skips or, alternatively, 
subjects with greater anagram-solving skills may have performed better and thus had little need for clues or skips.

Nevertheless, content analyses of subjects' counterfactuals were conducted, in which mentions of clues and skips were coded. Subjects who mentioned using fewer clues were given a score of 1 , those not mentioning clues were scored as 2 , and those mentioning greater use of clues were scored as 3 . This coding strategy was intended to create a dimensional analog for parametric analyses. Similar codings were derived for skips. These mentions of clues and skips were assessed using 2 (direction) $\times 2$ (structure) ANOVAs. Subjects in the upward condition were more likely to consider using fewer clues $(M=1.86)$ than those in the downward condition $(M=2.14), F(1,56)=4.17, p<.05$. The effect of structure was not reliable, nor were any effects in analyses of skips. Thus, encouraging people to consider upward counterfactuals improved their performance, and also led them to focus on restraint in clue use.

Mentions of clues and skips in subjects' counterfactual thoughts were then correlated with subjects' change in use of clues and skips from the first to second set (i.e., the number of clues selected in set 1 was subtracted from the number of clues selected in set 2, etc.). Mentions of using more clues were related to behavioural increases in clue use, $r(58)=.26, p<.05$. Mention of skips was unrelated to skip use, however, $r(58)=.01$, ns.

An ANOVA of the clue change scores with direction and structure as factors revealed a marginal effect of direction, $F(1,56)=3.19, p=.08$, and significant main effect of structure, $F(1,56)=10.54, p<.01$. Upward counterfactuals tended to result in decreased clue use $(M=-.53)$, whereas downward counterfactuals tended to elicit increased clue use $(M=.41)$. Additive 
counterfactuals resulted in lesser clue use $(M=-.93)$ than subtractive counterfactuals $(M=.83)$. It seems, then, that both upward and additive counterfactuals can enhance performance, and that they also reduced the use of clues. Thus, clue use seems to represent one avenue by which counterfactuals affect performance. The experimental elicitation of upward and additive counterfactuals increased thoughts focusing on restraint in clue use, which was in turn related to actual clue use in the second set of anagrams. Finally, this clue use was related to performance.

\section{Attributional Ratings}

The 4 attributional ratings were examined separately using $2 \times 2 \times ?$ ANOVAs. The only ANOVA for which an effect was significant was the analysis of task difficulty ratings. A 2-way interaction between counterfactual structure and SE was found, $F(1,52)=14.99, p<.01$. LSE subjects found the anagrams to be more difficult $(M=6.29)$ than HSE subjects $(M=4.31)$ after generation of additive counterfactuals, $t(52)=3.03, p<.01$. However, HSE subjects found the anagrams to be more difficult $(M=6.19)$ than LSE subjects $(M=4.79)$ following generation of subtractive counterfactuals, $t(52)=2.14, p<.05$. This interaction does not seem readily interpretable. No other effects were significant in this analysis.

\section{Correlations}

Correlations are presented in Tables 11 and 12. Consistent with the ANOVA findings, structure-DV was significantly correlated with total score improvement, $r(58)=.35, p<.01$ and with improvement in anagrams solved, 


\section{Table 11}

Correlations between counterfactual direction, structure, affect, expectancy ratings, and performance

\begin{tabular}{lcccccc}
\hline & ST & MS & DR & EX & TS & NC \\
\hline Direction-DV & .09 & -.05 & -.11 &. .01 & $.22^{*}$ & .10 \\
Structure-DV & & .02 & .01 & $.24^{*}$ & $.35^{* *}$ & $.23^{*}$ \\
Mood Scale & & $.62^{* *}$ & -.14 & $-.28^{*}$ & $-.29^{*}$ \\
Disappointed/Relieved & & & $-.27^{*}$ & $-.26^{*}$ & $-.31^{* *}$ \\
Expectancy Rating & & & & .18 & .19 \\
TS & & & & & $.88^{* *}$ \\
\hline
\end{tabular}

Note. For Direction-DV, higher numbers indicate more frequent upward counterfactuals; ST = Structure-DV (higher numbers indicate more frequent additive counterfactuals); $\mathrm{MS}=$ mood scale (for both affective measures, higher numbers indicate more positive affect); DR = disappointed-relieved rating; $T S=$ improvement in performance (total score); $\mathbf{N C}=$ improvement in performance (number of anagrams correctly solved). $N=60$ (control subjects were not included in this analysis).

${ }^{*} p<.05 ;{ }^{* *} p<.01$. 
Table 12

Correlations between counterfactual direction. structure, affect, and attributional ratings

\begin{tabular}{|c|c|c|c|c|c|c|c|}
\hline & ST & MS & DR & $\mathbf{R} 1$ & $\mathbf{R} 2$ & $\mathbf{R 3}$ & $\mathbf{R 4}$ \\
\hline Direction-DV & .09 & -.05 & -.11 & .16 & .10 & -.02 & .15 \\
\hline Structure-DV & & -.02 & .01 & .04 & .00 & -.10 & -.02 \\
\hline Mood Scale & & & $.62^{* *}$ & .05 & -.11 & .17 & -.16 \\
\hline \multicolumn{2}{|c|}{ Disappointed-Relieved } & & & $-.26^{*}$ & -.10 & .01 & -.21 \\
\hline R1 & & & & & .05 & $.37^{* *}$ & $.25^{*}$ \\
\hline $\mathbf{R} 2$ & & & & & & .11 & -.18 \\
\hline $\mathbf{R 3}$ & & & & & & & .10 \\
\hline
\end{tabular}

Note. For Direction-DV, higher numbers indicate more frequent upward counterfactuals; $\mathbf{S T}=$ Structure-DV (higher numbers indicate more frequent additive counterfactuals); DR = disappointed-relieved affect measure; $M S=$ mood scale (for both affective measures, higher numbers indicate more positive affect); $R 1$ = rating of personal responsibility; $R 2$ = rating of responsibility of others; $\mathbf{R 3}=$ rating of outcome controllability; $\mathbf{R} 4$ = rating of outcome avoidability (for all attributional ratings, higher numbers indicate more of the specified quality). $N=60$ (control subjects were not included in this analysis). ${ }^{*} p<.05 ;{ }^{* *} p<.01$. 
$r(58)=.23, p<.05$. Thus, additive structures were associated with greater improvement. Structure was also related to expectancy, $r(58)=.24, p<.05$. Although the ANOVA of performance expectancy ratings yielded no reliable effects, this correlational internal analysis suggests that additive counterfactuals are indeed associated with heightened expectancies for future success.

Unlike the first two experiments, counterfactual direction-DV was unrelated to affect. Interestingly, however, the affect measures were related to performance. As can be seen in Table 11, both the mood scale and the disappointed-relieveci measure were negatively correlated with the two indices of improvement in performance. For the mood scale, $r \mathrm{~s}(58)=-.28$ and -.29 for total score improvement and number solved improvement, respectively (both $p s<.05$ ). For the disappointed-relieved measure, $r s(58)=-.26$ and $-.31, p s<.05$ and .01 , for total score improvement and number solved improvement, respectively. Thus, more negative affect was associated with greater improvement. The disappointedrelieved measure was also negatively related to expectancy, such that people who felt more disappointed expected to do better on the second anagram task, $r(58)=$ $-.27, p<.05)$.

Unlike Experiment 2, direction-DV was unrelated to ratings of personal responsibility. The disappointed-relieved measure was negatively related to ratings of personal responsibility, such that people who felt more disappointed also felt more responsible for their performance on the first task $(r 58]=-.26, p<.05)$. No other correlations involving attributional ratings proved informative. 
Discussion

Experiment 3 was designed to provide further support for the preparative function that specific counterfactuals subtypes might serve. Using performance on a skills-based task as the dependent measure, significant effects of counterfactual direction and structure were noted, confirming the principal hypotheses. Contrary to expectations, however, the effect of direction on affect was not reliable.

Further support was provided for the hypothesis that upward as opposed to downward counterfactuals can in particular serve a preparative function. Subjects who were directed to generate the former type of counterfactuals demonstrated greater improvement in performance on the anagram task than subjects considering downward possibilities. Downward counterfactual generation produced a mean level of improvement almost identical to the no-counterfactual control condition. Although the contrast between the upward and the control condition fell short of significance, it would still seem to be the case that, consistent with Experiment 2, the main effect for direction is largely the result of the performance-enhancing effect of upward counterfactuals.

Another finding was that HSE people performed better than LSE people. Of course, the causal interpretation of any individual difference effect is necessarily ambiguous. This is particulariy true here, because the SE scale was completed after both anagram tasks. HSE people may be better motivated and better skilled at such verbal skills tasks, but it is also possible that people who performed poorly also scored lower on the SE measure as a result of their disappointment.

As predicted but unlike Experiment 2, a significant main effect for 
counterfactual structure was found. Consideration of additive counterfactuals resulted in greater improvement than consideration of subtractive counterfactuals. Control group analyses indicated that this main effect was due largely to the performance-enhancing effect of additive counterfactuals and not to any performance-retarding effect of subtractive counterfactuals. The fact that the structure dimension was significant as a main effect in Experiment 3 but not 2 suggests that additive counterfactuals exert their preparative effect in a more highly circumscribed, domain-specific manner than upward counterfactuals. Thus, although the latter may induce broader effects on a variety of intentions, the effects of the former might be more specific, both conceptually and temporally. Experiment 3 thus provides further evidence for the hypothesized pathway linking counterfactuals to behaviour, theorized to be the basis of the preparative function. It was posited that upward and additive counterfactuals (e.g., "If only I had done $X$, things would have been better") may be especially likely to be converted into prefactuals (e.g., "If I do X, things may turn out better in the future"). Such prefactual thoughts have previously been shown to heighten intention: to perform, as well as the actual performance of, relevant successfacilitating behaviours (e.g., Anderson, 1983; Gregory et al., 1982; Sherman et al., 1981). Experiment 2 showed that a counterfactual manipulation could have an effect on behavioural intentions. Experiment 3 extends this finding by showing that both upward and additive counterfactuals can have an effect on overt behaviour. Moreover, an analysis of use of clues by subjects suggested that one avenue by which these counterfactuals affected performance was through the realization that restraint in clue use might enhance one's score. The counterfactual manipulations influenced the mention of clue use in the 
counterfactuals that subjects recorded, and these mentions were related to actual clue use, which was in turn related to performance. It is important to note also that the present experiment constitutes the first empirical documentation of behavioural consequences of counterfactual thinking. One initial concern raised in the Introduction was that counterfactual thoughts are epiphenomenal and hence inconsequential. The present findings demonstrate quite clearly that this statement is untenable.

The predicted effect of counterfactual direction on affect was not found. A 3-way interaction involving SE and the two manipulated variables was significant but uninterpretable. One possible explanation for the null effect of direction an affect is that the laboratory anagram-solving task was simply not sufficiently egoinvolving or ego-threatening to produce counterfactual thought-mediated affective consequences. The previous two experiments were based on retrospective selfreports, which perhaps involved more intense or more ego-threatening affective experiences. In Experiment 1, many subjects recalled extremely upsetting personal events (e.g., the breakup of a relationship). In Experiment 2, subjects recalled a particularly disappointing performance on an examination. Because the experiment was deliberately conducted in late November, the overwhelming majority recalled fall midterm exams -- subjects' first experiences with universitylevel testing. Unfulfilled expectations of academic success likely represent a particularly painful source of dismay for many first-year university students. Generating downward alternatives to these events may result in meaningful improvements in mood. In contrast, Experiment 3 involved failure on a task that was relatively less personally important. The consideration of upward versus downward alternatives to this outcome may have held little affective significance 
for subjects at a personal level. Future research may determine whether it is these situational differences that can account for the failure in Experiment 3 to replicate the affective findings of Experiments 1 and 2.

Interestingly, affect was significantly related to performance. Those expressing more negative feelings subsequently improved more on the second anagram set. Thus, a motivational mechanism in which people who feel worse try harder may have been in effect. Because the counterfactual manipulations had no impact on affect, however, this mechanism probably played no mediating role in the link between counterfactuals and performance. 


\section{CHAPTLR V \\ GENERAL DISCUSSION}


Taken together, the results of the three experiments provide general support for the hypothesis that counterfactual thinking can serve both an affective and a preparative function. In Experiment 1, direction but not structure influenced affect, such that consideration of downward counterfactuals resulted in more positive affect than consideration of upward counterfactuals. In Experiment 2, direction but not structure influenced affect in the same manner as in Experiment 1. The inclusion of a no-counterfactual control group indicated that this main effect was largely the result of the positive affect-enhancing effect of downward counterfactuals. Direction but not structure influenced intention ratings, such that upward counterfactual generation resulted in heightened intentions to perform success-facilitating behaviours. In Experiment 3, subjects engaged in a computer-administered anagram task. Although the affective consequences of counterfactual generation were not significant, both direction and structure influenced performance: upward as well as additive counterfactual generation resulted in greater improvement on the anagram task, relative to downward and subtractive counterfactual conditions. Overall, counterfactuals of specific subtypes can make us feel better, and counterfactuals of other subtypes can facilitate our improvement in the future. The purpose of this General Discussion is to consider some of the theoretical implications of these findings.

\section{The Affective Function}

The affective function of counterfactuals was demonstrated in Experiments 
1 and 2. Downward counterfactuals caused more positive affect than upward counterfactuals. This finding is consistent with previous theoretical explication (Johnson \& Sherman, 1990; Taylor \& Schneider, 1989) and significantly extends the only extant empirical demons ration (Markman et al., 1993). Ir. Experiment 2, the use of a control group permitted an even greater specification of this finding. The main effect for direction was found to be mainly a function of the positive affect-enhancing effect of downward counterfactuals. Relative to control subjects who generated no counterfactuals, consideration of upward counterfactuals created no more negative affect.

This latter finding may seem surprising. Popular wisdom would certainly assert that upward counterfactuals hold the power to sadden and depress us. This sentiment is central to John Greenleaf Whittier's famous aphorism, "For all sad words of tongue and pen, the saddest are these: It might have been". Moreover, this idea is consistent, albeit indirectly, with previous counterfactual findings (e.g., Gleicher et al., 1990; Johnson, 1986; Kahneman \& Miller, 1986; Landman. 1987). In these studies, manipulations that heightened counterfactual availability following negative outcomes (e.g., via the salience of exceptional vs normal antecedents, or act vs non-act antecedents) also tended to amplify negative affect. Thus, greater frequency of generation of counterfactuals, which were likely predominantly upward (since generation followed a negative outcome, e.g., Markman et al., 1993), produced greater regret and disappointment than conditions in which counterfactual alternatives were less available. However, relative deprivation research, which examines the antecedents and consequences of feelings of personal deprivation with respect to one's life situation, has tended to show that consideration of how things might have be better (i.e., upward counterfactual 
comparisons) does not engender greater dissatisfaction (or other negative affect) relative to a no-counterfactual control condition (Folger, 1984; Folger, Rosenfield, Rheaume, \& Martin, 1983; Folger, Rosenfield, \& Robinson, 1983; Hafer \& Olson, 1989). Rather, the effect of these upward counterfactuals (termed "referent cognitions" within this literature) tends only to occur in combination with other variables, resulting in significant interaction effects.

Of course, the null effect of upward counterfactuals on affect found in Experiment 2 may be simply artifactual. Specifically, since affect ratings were made following the recollection of a negative life experience, and since all ratings therefore fell into the regative 'alf of the rating scale, a simple floor effect might have precluded any negative effects of upward counterfactual comparisons. Another possibility is that any negative affect aroused by upward counterfactuals was mitigated somewhat because such thoughts may also raise hope for future betterment. which would tend to engender positive affect (e.g., Boninger et al., 1992: Buunk et al.. 1990).

Differences in the nature of the upward counterfactual thoughts themselves may also account for the discrepancy between the present versus past experimental finuings. It may be that there is something particularly distressing. poignant, and unnerving about counterfactuals based on the removal of negative exceptional antecedents, relative to those counterfactuals focusing on more normal antecedentc. If I perform poorly at a job interview. I may feel much more upset if my focus is on having spilt a glass of water on the interviewer (e.g.. "Things would have gone so much better if only I hadn't asked for the water!") than if I focus on more normal, typical aspects of such a situation. Perhaps the negative affective consequences of upward counterfactuals are limited to, or at least greatly 
enhanced by, cases where something atypical (and negative) preceded the outcome. It should be pointed out that, by definition, exceptional events are rare. Yet, counterfactual thinking seems to be ubiquitous. It seems safe to conclude that the vast majority of counterfactual thoughts generated on an everyday basis therefore invoive mutations of relatively typical antecedents, and that it was these that were primarily sampled in Experiment 2. Perhaps these normality-based counterfactuals typically do not lead to more negative affect even when the direction of comparison is upward, or do so only in a very weak, transient fashion. Thus, although it is possible that the null effect of upward counterfactuals on affect reflects a floor effect, there exist compelling conceptual reasons to accept it, pending further empirical elucidation, as real. Nevertheless, these experiments do provide compelling evidence that downward counterfactuals can cause more positive affect, lending important support to previous suggestions that people strategically use such counterfactual thoughts to make themselves or others feel better (Johnson \& Sherman, 1990; Markman et al., 1993).

\section{The Preparative Function}

Experiments 2 and 3 provided direct support for the contention that upward as opposed to downward counterfactuals, and additive as opposed to subtractive counterfactuals, can in particular serve the function of preparing for future betterment. Such preparation was conceptualized in terms of improvement and avoidance of the recurrence of past failure. The present findings extend the evidence provided by previous research.

The general strategy of past examinations of the preparative function of counterfactual thinking has been based on situational manipulations. That is, 
situations were identified in which cognitive processes serving a preparative function would be likely to occur (i.e., situations in which they would be useful). Markman et al. (1993) manipulated outcome valence and outcome repeatability, reasoning that negative as opposed to positive outcomes, and repeating as opposed to nonrepeating outcomes, represent situations in which functional counterfactuals would be most useful and, hence, most likely. Upward counterfactuals were indeed more frequent than downward counterfactuals in these situations. In a similar fashion, Roese and Olson (1993c) manipulated outcome valence and outcome frame, reasoning that negative as opposed to positive outcomes, and outcomes framed by negative as opposed to positive past histories, represent situations in which counterfactuals serving the preparative function would predominate. As predicted, additive counterfactuals were more frequent than subtractive counterfactuals in these situations. However, these demonstrations are necessarily indirect. Although it makes intuitive sense that processes that prepare one for the future should occur in situations where they are especially useful, in the absence of direct evidence regarding the consequences of engaging in these processes, they could also be afunctional and represent nonadaptive cognitive responses.

The present experiments were designed to provide more direct evidence. The approach taken was to manipulate the presence versus absence of the particular cognitive process thought to be functional, then to observe consequences that can be defined in terms of its functional value. In so doing, the dependent variables of Markman et al. (1993) and Roese and Olson (1993c) were converted into independent variables. As expected, upward as opposed to downward, and additive as opposed to subtractive counterfactuals were shown to 
causally improve performance. Taken together, the present findings, along with the two previous indirect demonstrations, provide convincing evidence that both upward and additive counterfactuals may be used strategically to improve future outcomes. The exact mechanism underlying these effects remains somewhat equivocal, however. Subjects' use of clues in the anagram task appeared to offer support for the hypothesized mechanism linking counterfactuals, prefactuals, intentions, and actions. That is, the counterfactual manipulations affected subjects' consideration of clue use (revealed by content analyses of the counterfactuals subjects recorded), which in turn was related to the actual use of clues in the second anagram set. Overall, restraint in clue use was associated with greater improvement. Identification of this one avenue by which counterfactuals led to improvement does not preclude the operation of other mechanisms, however. An alternative mechanism might be that upward counterfactuals in particular, and perhaps additive counterfactuals to a lesser extent, offer individuals hope for the future (cf. Buunk et al., 1990; Taylor, Buunk, \& Aspinwall, 1990). Optimistic appraisals of future possibilities may in themselves be a motivating force, causing subjects simply to try harder on subsequent tasks. In the absence of more direct evidence, this alternative explanation remains plausible.

In more general terms, these findings are consistent with research on goalsetting. Within this tramework, the term motivation takes on a broader, more colloquial definition as intentional achievement-striving (e.g., Kleinbeck \& Schmidt, 1990). Forming specific goals and planning how to meet those goals are routinely found to enhance performance on a range of tasks (e.g., Locke \& Latham, 1990). Moreover, "reflection" on past outcomes has been described as a useful strategy for improving decision-making and future performance (e.g., 
Goodhart, 1986). This research tends to be somewhat atheoretical, yet it is clearly consistent with the more conceptually incisive research to be found in the prefactual literature (e.g., Campbell \& Fairey, 1985; Sherman et al., 1981; see also Showers, 1992). In these studies, a general focus on positive as opposed to negative future possibilities, combined with the generation of scenarios describing success, has been found to improve subsequent performance. A counterfactual framework could provide greater theoretical precision within the goal-setting literature. Specification of subtypes of conditional statements, both counterfactual and prefactual, might prove to be an effective means of enlarging the theoretical scope and sophistication of goal-setting research, permitting greater predictive accuracy as well as more effective interventions.

The question of the relation between the two variables of counterfactual direction and structure was not explicitly examined in the present research. These two variables were conceptualized as orthogonal, permitting the specification of a $2 \times 2$ matrix of counterfactual subtypes. However, my previous research suggests that the two variables may be weakly related (average $r<.25$ ). Thus, upward counterfactuals may co-occur with additive counterfactuals, although not strongly. This would be consistent with the findings that both upward and additive counterfactuals tend to predominate after failure (Markman et al., 1993; Roese \& Olson, 1993c), though their respective patterns diverge after success. In one study that assessed the spontaneous generation of these four subtypes following success or failure, cell frequencies within the $2 \times 2$ matrix did not differ reliably (Roese $\&$ Olson, 1993a). Within the present study, the independence of the two variables was enforced by the nature of the manipulations (subjects were instructed to record only the counterfactual subtype specified). However, it could be argued 
that some subtypes represent more natural, common associations between direction and structure (e.g., upward combined with additive). These subtypes should more easily come to mind, and subjects might have recorded these with greater frequency when solicited relative to the solicitation of less easily conceived subtypes. However, the tests of the manipulation showed only the expected main effects and no interactions. Further, the correlations between direction and structure tended to be low and nonsignificant $(r s=.24, .22, .09$, for Experiments 1,2 , and 3, respectively). ${ }^{13}$ Thus, the present 3 experiments provide further support for the conceptual independence of the dimensions of direction and structure.

The findings regarding the preparative function may reflect a more basic human propensity to view the past using counterfactual inferences. Recent research has shown that causal ascriptions for past outcomes may be based on counterfactual inferences (Lipe, 1991; Wells \& Gavanski, 1989). Hindsight biased judgments of inevitability or "knowing it all along" may also be based on counterfactual inferences (see Miller \& Turnbull, 1990). In fact, those who look at the past professionally (e.g., historians, political scientists) routinely use counterfactual comparisons as a proxy experimental test (i.e., as a means of testing the causal importance of various historical events; see Fearon, 1991). Such evidence suggests that counterfactual reasoning may represent a basic mechanism by which people perceive reality.

15 It is important to note that the correlations between direction and structure presented in this dissertation represent the relation between two difference scores. Such correlations may be artifactually inflated due to statistical bias (e.g., Cronbach \& Furby, 1970; Gardner \& Neufeld, 1987). Thus, the correlations presented are likely overestimates of the population parameters. 
The Interplay Between Affective and Preparative Processes

Markman et al. (1993) focused their discussion of the functional role of counterfactual thinking exclusively on the dimension of direction. One of their findings was the prominence of upward counterfactuals following outcomes framed as failures. These authors noted that people may face a trade-off between the benefits (i.e., preparation for the future) and the drawbacks (i.e., immediate feelings of dissatisfaction) of forming upward counterfactual thoughts (see also Boninger et al., 1992).

The present research suggests that conceptualizing counterfactual functionality as a "trade-off" may be unwarranted. Rather, it may be possible for people to have their cake and eat it too, so to speak. Both the preparative as well as the affective value of counterfactual thinking may be maximized simultaneously simply by increasing the frequency of considering both upward and downward alternatives. Upward counterfactuals can help one to prepare for the future, but may not carry any negative affective consequences. Similarly, downward counterfactuals can increase positive affect, but according to the present findings, they do not mitigate preparativeness. Thus, the mere increase in vigour of counterfactual thinking may be personally beneficial in two distinct ways, while not involving any so-called trade-off.

The trade-off may emerge, however, in cases where the counterfactual is fabricated from the removal of an exceptional antecedent element. As noted previously, it may be these specific subcases of upward counterfactuals that hold the power truly to depress and obsess us. Recent research by Boninger et al. (1992) suggests that directing people's thoughts away from "what might have been" directly to "what may be next time" may cause people to experience less regret 
and self-blame with respect to an aversive event suffered in the past. This effect occurred only for those individuals who were dispositionally less likely to consider the future consequences of their actions. As with the bulk of the extant counterfactual research, this research focused on counterfactuals constructed from exceptional antecedents.

One of the potentially surprising conclusions that can be drawn from the present body of research is the lack of any evidence for the moderating role of self-esteem in either the affective or the preparative function. Previous research has shown that HSE individuals are better able than LSE individuals to manipulate counterfactual thoughts in a self-serving and, hence, affectively selfenhancing manner (Roese \& Olson, 1993b). Evidence from the social comparison literature suggested the hypothesis that HSE persons may be more likely than LSE persons to report positive affect in general, but that this difference might be even greater after consideration of upward comparisons (Buunk et al., 1990; Taylor et al., 1990). Thus, it was hypothesized that self-esteem would interact with direction, such that HSE persons would report more positive affect than LSE persons following the generation of downward counterfactuals, but that the magnitude of this self-esteem difference would be much greater following the generation of upward counterfactuals. This interaction effect was not significant in any of the experiments, nor was the expected main effect for self-esteem. One possible explanation is that the self-esteem measure (the TSBI), which focuses on the social and interpersonal aspects of self-esteem, was inappropriate for these purposes. Perhaps a measure of global self-esteem (e.g., the Rosenberg scale; see Blascovich \& Tomaka, 1991) might have illuminated more interesting patterns of findings. 
These results could be interpreted as consistent, however, with the extant literature on self-esteem differences. It may be the case that the mechanism by which downward counterfactuals increase positive affect is relatively invariant across individuals. That is, virtually all people tend to feel better after considering how things might have turned out worse. The differences between HSE and LSE persons may only emerge in the strategic use of this mechanism as a means of self-enhancement. This being the case, self-esteem differences would not be expected in experiments in which counterfactuals are manipulated and affect assessed as a dependent measure. Rather, self-esteem differences might be illuminated by research in which the strategic use (i.e., spontaneous generation) of upward and downward counterfactuals is measured as a function of self-esteem, and perhaps also of outcome valence or involvement (i.e., variables which would likely influence the personal desire to self-enhance) (cf. Brown, Collins, \& Schmidt, 1988; Roese \& Olson, 1993b).

\section{Conceptual Limitations of the Present Research}

Several limitations should be noted. First, these experiments examined first-year university students only, whose responses may not be representative of North Americans in general. Moreover, counterfactual thinking may be constrained by cultural and linguistic factors (e.g., Au, 1983; Bloom, 1981; Liu, 1985), none of which was considered in the present work. Second, the tasks involved were somewhat trivial. In particular, the anagram task used in Experiment 3 may represent a comparatively unimportant setting in which to observe counterfactual effects. Although the preparative findings may well be general, it is also possible that such effects are limited to tasks that are relatively 
less ego-involving. The effects may also be limited to achievement contexts.

Third, a demand interpretation of some of the findings is possible. For instance, the finding that downward counterfactuals elicit more positive affect may have been due to the demand associated with the linguistic stem "at least". Because these two words often preface attempts by others to make us feel better, subjects may have assumed that this was the focus of the study, and responded accordingly (i.e., with ratings indicating that they indeed felt better). On a more general level, the fact that the affective findings of Experiments 1 and 2 did not generalize to Experiment 3, and that the main effect of structure in Experiment 3 was not evident in Experiment 2, underline the need for further empirical examination.

The foregoing theoretical development has focused on the ways in which counterfactual thoughts may be beneficial for the individual at a global level. Implicit has been the assumption that people desire to maximize benefits and minimize failure, and will engage in behaviour appropriate to these desires. Although this assumption forms the basis of many useful descriptions of human behaviour, it is certainly not the case that people always behave in ways that are beneficial and self-enhancing. Sometimes the generation of counterfactual thoughts may follow patterns that are afunctional; on other occasions, counterfactual usage may be counterproductive.

Some counterfactual thoughts may serve no useful purpose at all. For example, I may wonder what life would be like if people were blimplike waterbreathers, or if Germany had won the Second World War. ${ }^{10}$ Thoughts of such a

16 Fiction inspired by the counterfactual possibility of a Nazi victory in the Second World War represents a burgeoning literary subgenre (e.g., Benford \& Greenberg. 1991; Harris, 1992). Not surprisingly, these tales might all be appropriately deemed "downward" counterfactuals. 
fleeting, ephemeral nature likely carry little cognitive or behavioural significance, and may appropriately be termed afunctional. In other cases, generation of counterfactual thoughts may be psychologically damaging and, hence, counterproductive. An endless replaying of past failures, restructured over and over again with minor alterations, might represent a counterfactual mechanism that promulgates depressive states. Previous descriptions of such a "ruminative" coping style have shown that individuals engaging in such thoughts are unlikely to take direct action to deal with their problems, are more vulnerable to chronic selfdirected affect, and ultimately perhaps, are at greater risk for depression (e.g., Wood, Saltzberg, Neale, Stone, \& Rachmiel, 1990). For example, Roese and Olson (1993b) found that LSE persons tend to show a self-denigrating pattern of counterfactual thinking, one that may induce more negative affect, perhaps in concert with other components of what Pyszczynski and Greenberg (1987) described as a depressive cognitive style.

Counterfactual thoughts may not only exacerbate negative affect, but they may also be structured in such a way that precludes or retards performanceenhancement. People who chronically generate counterfactuals that are selfprotective (e.g., that focus on external causal antecedents of failure) may be less likely to identify means of improving themselves in the future. In the rush to blame others for failure, these individuals may not be able to use counterfactuals preparatively. Thus, it should be emphasized that the present theoretical analysis by no means embraces all possible counterfactual uses or effects, but is intended merely to illuminate the smaller subset of cognitions that may be interpreted as globally beneficial. 
Conclusion

The consideration of what "might have been" and of alternative yet "possible" worlds has long fascinated philosophers. Many such explorations have focused predominantly on the logical-semantic structure of counterfactual conditionals (e.g., Kvart, 1986; Lewis, 1973; Reisenbach, 1976). However, on a more uniquely human level, others have suggested that the ability to "project thought and imagination into the 'if-ness'; into the free conditionalities of the unknown" is an essential precursor to human consciousness, action, and civilization. "It is the master nerve," wrote Steiner (1975, p. 217) of the human ability to conceive of both what might have been and what may yet be. Three laboratory experiments provided evidence that counterfactuals of specific subtypes can make individuals feel better, and that counterfactuals of other subtypes can facilitate individual improvement in the future. Thus, counterfactual thoughts may indeed function as that master nerve, allowing individuals to better themselves and their surroundings in globally meaningful ways. 


\section{REFERENCES}


Anderson, C. A. (1983). Imagination and expectation: The effect of imagining behavioral scripts on personal intentions. Joumal of Personality and Social Psychology, 45, 293-305.

Au, T. K. (1983). Chinese and English counterfactuals: The Sapir-Whorf hypothesis revisited. Cognition, 15, 155-187.

Benford, G., \& Greenberg, M. H. (Eds.) (1990). What might have been? Volume 3: Altemate wars. New York: Bantam Spectra.

Blascovich, J., \& Tomaka, J. (1991). Measures of self-esteem. In J. P. Robinson, P. R. Shaver, \& L. S. Wrightsman (Eds.), Measures of personaliny and social psychological animudes (pp. 115-160). San Diego, CA: Academic Press.

Bloom, A. H. (1981). The linguistic shaping of thought: A study in the impact of language on thinking in China and the West. Hillsdale, NJ: Erlbaum.

Boninger, D. S., Gleicher, F., \& Strathman, A. (1992). Counterfactual thinking: From what might have been to what may be. Unpublished manuscript.

Boring, E. G. (1957). A history of experimental psychology (2nd ed.). New York: Appleton Century Crofts.

Bouts, P., Spears, R., \& van der Pligt, J. (1992). Counterfactual processing and the correspondence between events and outcomes: Normality versus value. European Joumal of Social Pyychology, 22, 387-396.

Brown, J. D., Collins, R. L., \& Schmidt, G. W. (1988). Self- -teem and direct versus indirect forms of self-enhancement. Joumal of Personuliny and Social Psychology, 55, 445-453.

Bulman, R. J., \& Wortman, C. B. (1977). Attribution of blame and coping in the "real world": Severe accident victims react to their lot. Joumal of Personality and Social Psychology. 35, 351-363.

Burgess, A. W.. \& Holmstrom. L. (1979). Rape: Crisis and recoveny. Bowie. MD: Brady. 
Buunk, B. P., Collins, R. L.. Taylor, S. E., VanYperen, N. W., \& Dakof, G. A. (1990). The affective consequences of social comparisons: Either direction has its ups and downs. Joumal of Persomality and Social Psychology, 59, 1238-1249.

Campbell, J. D.. \& Fairey, P. J. (1985). Effects of self-esteem, hypothetical explanations, and verbalization of expectancies on future performance. Joumal of Personality and Social Psychology, 48, 1(197-1111.

Carmer, S. G., \& Swanson, M. R. (1973). An evaluation of ten pairwise multiple comparison procedures by Monte Carlo methods. Jotmal of the American Statistical Association, 68, 66-74.

Carroll, J. S. (1978). The effect of imagining an event on expectations for the event: An interpretation in terms of the availability heuristic. Joumal of Experimental Social Psychology, 14, 88-96.

Crocker, J.. Thompson, L. L.. McGraw, K. M.. \& Ingerman. C. (1987). Downward comparison prejudice and evaluations of others: Effects of selfesteem and threat. Joumal of Personality and Social Psychology, 52, 907 916.

Cronbach, L. J., \& Furby, L. (1970). How should we measure "change" - Or should we? Psychological Bullerin, 74, 68-80.

Dunning, D., \& Parpal, M. (1989). Mental addition and subtraction in counterfactu: $;$ reasoning: On assessing the impact of actions and life events. Joumal of Personaliny and Social Psychology, 57, 5-15.

Epstein, S., Lipson, A., Holstein, C., \& Huh, E. (1992). Irrational reactions to negative outcomes: Evidence for two conceptual systems. Joumal of Persomality and Social Psychology, 62, 32s-339.

Esses, V. M., Haddock, G., \& Zanna, M. P. (in press). The role of mood in the expression of intergroup stereotypes. In M. P. Zanna \& J. M. Olson (Eds.), The psychology of prejudice: The Ontario Symposium (Vol. 7). Hillsdale, NJ: Eribaum.

Fearon, J. D. (1991). Counterfactuals and hypothesis testing in political science. World Politics, 43, 169-195.

Festinger, L. (1954). A theory of social comparison processes. Human Relations, 7, 117-140. 
Flaubert. G. (1857/1950). Madame Bovary (trans. A. Russell). London: Penguin.

Folger, R. (1984,. Perceived injustice, referent cognitions, and the concept of comparison level. Representative Research in Social Psychology, 14, 88-108.

Folger, R., Rosenfield, D., Rheaume, K., \& Martin, C. (1983). Relative deprivation and referent cognitions. Joumal of Experimental Social Psychology, 19, 172-184.

Folger, R., Rosenfield, D., \& Robinson, T. (1983). Relative deprivation and procedural justifications. Joumal of Personality and Social Psychology, 45, 268-273.

Gardner, R. C., \& Neufeld, R. W. J. (1987). Use of the simple change score in correlational analyses. Educational and Psychological Measurement, 47, 849864.

Gavanski, I., \& Wells, G. L. (1989). Counterfactual processing of normal and exceptional events. Journal of Experimental Social Psychology, 25, 314-325.

Gilhooly, K. J., \& Johnson, C. E. (1978). Effects of solution word attributes on anagram difficulty: A regression analysis. Quarterly Joumal of Experimental Psychology, 30, 57.70.

Girotto, V., Legrenzi, P., Rizzo, A. (1991). Event controllability in counterfactual thinking. Acta Psychologica, 78, 111-133.

Gleicher, F., Kost, K. A., Baker, S. M., Strathman, A. J., Richman, S. A., \& Sherman, S. J. (1990). The role of counterfactual thinking in judgments of affect. Personality and Social Psychology Bulletin, 16, 284-295.

Goodhart, D. E. (1986). The effects of positive and negative thinking on performance. Joumal of Personality and Social Psychology, 51, 117-124.

Gregory, W. L., Burroughs, J. W., \& Ainslie, F. M. (1985). Self-relevant scenarios as an indirect means of attitude change. Personality and Social Psychology Bulletin, 11, 425-444.

Gregory, W. L., Cialdini, R. B., \& Carpenter, K. M. (1982). Self-relevant scenarios as mediators of likelihood estimates and compliance: Does imagining make it so? Joumal of Personality and Social Psychology, 43, 89. 99.

Hafer, C. L., \& Olson, J. M. (1989). Beliefs in a just world and reactions to 
personal deprivation. Joumal of Personality, 57, 799-823.

Hairis, R. (1992). Fatherland. London: Arrow Books.

Heider, F. (1958). The psychology of interpersonal relations. New York: Wiley.

Helmreich, R., \& Stapp, J. (1974). Short forms of the Texas Social Behavior Inventory (TSBI), an objective measure of self-esteem. Bu!letin of the Psychonomic Society, 4, 473-475.

Hemphill, K. J., \& Lehman, D. R. (1991). Social comparisons and their affective consequences: The importance of comparison dimension and individual difference variables. Joumal of Social and Clinical Psycholugy, 10, 372-394.

Hofstadter, D. R. (1979). Gödel, Escher, Bach: An etemal golden braid. New York: Vintage Books.

Hofstadter, D. R. (1985). Metamagical themas: Questing for the essence of mind and pattem. New York: Basic Books.

Jellison, J. M., \& Green, J. (1981). A self-presentation approach to the fundamental attribution error: The norm of internality. Joumal of Personality and Social Psychology, 40, 643-649.

Johnson, J. T. (1986). The knowledge of what might have been: Affective and attributional consequences of near outcomes. Personality and Social Psychology Bulletin, 12, 51-62.

Johnson, M. K., \& Sherman, S. J. (1990). Constructing and reconstructing the past and the future in the present. In E. T. Higgins \& R. M. Sorrentino (Eds.). Handbook of motivation and cognition: Foundations of social behavior (Vol. 2, pp. 482-526). New York: Guilford Press.

Kahneman, D., \& Miller, D. T. (1986). Norm theory: Comparing reality to its alternatives. Psychological Review, 93, 136-153.

Kahneman, D., \& Tversky, A. (1982a). The psychology of preferences. Scientific American, 246(1), 160-173.

Kahneman, D.. \& Tversky, A. (1982b). The simulation heuristic. In D. Kahneman, P. Slovic, \& A. Tversky, (Eds.), Judgment under uncertainty: Heuristics and biases (pp. 201-208). New York: Cambridge University Press. 
Kahneman, D., \& Varey, C. A. (1990). Propensities and counterfactuals: The loser that almost won. Joumal of Personaliyy and Social Psychology, 59, 1101-1110.

Katz, D. (1960). The functional approach to the study of attitudes. Public Opinion Quarterty, 24, 163-204.

Kelley, H. H. (1971). Attributions in social interactions. Morristown, NJ: General Learning Press.

Kirkpatrick, L. A., \& Epstein, S. (1992). Cognitive-experiential self-theory and subjective probability: Further evidence for two conceptual systems. Journal of Personality and Social Psychology, 63, 534-544.

Kleinbeck, U., \& Schmidt, K. H. (1990). The translation of work motivation into performance. In U. Kleinbeck, H. H. Quast, H. Thierry, \& H. Hacker (Eds.), Work motivation (pp. 27.39). Hillsdale, NJ: Erlbaum.

Kvart, I. (1986). A theory of counterfactuals. Indianapolis, IN: Hackett Publishing.

Landman, J. (1987). Regret and elation following action and inaction: Affective responses to positive versus negative outcomes. Personaliny and Social Psychology Bulletin, 13, 524-536.

Langer, E. J. (1975). The illusion of control. Joumal of Personality and Social Psychology, 32, 311-328.

Lazarus, R. S., \& Folkman, S. (1984). Stress, appraisal, and coping. New York: Springer.

Lewis, D. K. (1973). Counterfactuals. Cambridge, MA: Harvard University Press.

Lipe, M. G. (1991). Counterfactual reasoning as a framework for attribution theories. Psychological Bulletin, 109, 456-471.

Liu, L. G. (1985). Reasoning counterfactually in Chinese: Are there any obstacles? Cognition, 21, 239-270.

Locke, E. A., \& Latham, G. P. (1990). A theory of goal setting and task performance. Englewood Cliffs, NJ: Prentice-Hall.

Mackie, J. L. (1974). Cement of the universe: A study of causation. London: Oxford University Press. 
Macrae, C. N. A tale of two curries: Counterfactual thinking and accident-related judgments. Personality and Social Psychology Bulletin, 18, 84-87.

Macrae, C. N., \& Milne, A. B. (1992). A curry for your thoughts: Empathic effects on counterfactual thinking. Personality and Social Psychology Bulletin, 18, 625-630.

Markman, K. D., Gavanski, I., Sherman, S. J., \& McMullen, M. N. (1993). The simulation of better and worse possible worlds. Joumal of Experimental Social Psychology. 29, 87-109.

McFarland, C., \& Ross, M. (1982). Impact of causal attributions on affective reactions to success and failure. Joumal of Personality and Social Psychology, 43, 937-946.

McGill, A. L., \& Klein, J. G. (1993). Contrastive and counterfactual thinking in causal judgment. Joumal of Personality and Social Psychology, 64, 897-905.

Miller, D. T., \& Gunasegaram. S. (1990). Temporal order and the perceived nutability of events. Implications for blame assignment. Joumal of Personality and Social Psychology, 59, 1111-1118.

Miller, D. T., \& McFarland, C. (1986). Counterfactual thinking and victim compensation: A test of norm theory. Personality and Social Psychology Bulletin, 12, 513-519.

Miller, D. T., \& Ross, M. (1975). Self-serving biases in the attribution of sausality: Fact or fiction? Psychological Bulletin, 82, 213-225.

Miller, D. T., Taylor, B., \& Buck, M. L. (1991). Gender gaps: Who needs to be explained? Joumal of Personality and Social Psychology, 6I, 5-12.

Miller, D. T., \& Turnbull, W. (1990). The counterfactual fallacy: Confusing what might have been with what ought to have been. Social Justice Research, 4, 1-19.

Miller, D. T., Turnbull, W., \& McFarland, C. (1989). When a coincidence is suspicious: The role of mental simulation. Joumal of Personality and Social Psychology, 57, 581-589.

Miller, D. T., Turnbull, W., \& McFarland, C. (1990). Counterfactual thinking and social perception: Thinking about what might have been. In M. P. Zanna (Ed.), Advances in experimental social psychology (Vol. 23, pp. 305-331). New York: Academic Press. 
Nosanchuk, T. A., \& Erickson, B. H. (1985). How high is up? Calibrating social comparison in the real world. Joumal of Personaliny and Social Psychology, 48, 624-634.

Parducci, A. (1968). The relativism of absolute judgments. Scientific American, 219(6), 84-90.

Paulhus, D. L. (1984). Two-component models of socially desirable responding. Joumal of Personaliny and Social Psychology, 46, 598-609.

Pipes, R. B., Bowers, M. A., Hilton, K. K., Mathews, L. S., Oates, D. F. (1991, August). Suspiciousness: The (lack of) role of mental simulation. Paper presented at the annual convention of the American Psychological Association, San Francisco.

Pyszczynski, T., \& Greenberg, J. (1987). Self-regulatory perseveration and the depressive self-focusing style: A self-awareness theory of reactive depression. Psychological Bulletin, 102, 122-138.

Reichenbach, H. (1976). Laws, modalities, and coumterfactuals. Los Angeles: University of California Press.

Roese, N. J. (1991). The coaching skills study (unpublished raw data), University of Western Ontario.

Roese, N. J., \& Olson, J. M. (1991, June). Self-esteem, motivation, and counterfactual thinking. Paper presented at the annual convention of the Canadian Psychological Association, Calgary.

Roese, N. J., \& Olson, J. M. (1992, June). The structural basis of coumerfactual thought. Paper presented at the annual convention of the Canadian Psychological Association, Quebec City.

Roese, N. J., \& Olson, J. M. (1993a, May). Outcome controllability and counterfactual thinking. Paper presented at the annual convention of the Canadian Psychologica! Association, Montreal.

Roese, N. J., \& Olson, J. M. (1993b). Self-esteem and counterfactual thinking. Joumal of Personality and Social Psychology, 65,199-206.

Roese, N. J., \& Olson, J. M. (1993c). The structure of counterfactual thought. Personality and Social Psychology Bulletin, 19, 312-319.

Sherman, R. T., \& Anderson, C. A. (1987). Decreasing premature termination 
from psychotherapy. Joumal of Social and Clinical Psychology, 5, 298-312.

Sherman, S. J., Cialdini, R. B., Schwartzman, D. F., \& Reynolds, K. D. (1985). Imagining can heighten or lower the perceived likelihood of contracting a disease: The mediating effect of ease of imagery. Personality and Social Psychology Bullerin, 11, 118-127.

Sherman, S. J., Skov, R. B., Hervitz, E. F., \& Stock, C. B. (1981). The effects of explaining hypothetical future events: From possibility to probability to actuality and beyond. Joumal of Experimental Social Psychology, 17, 142158.

Sherman, S. J., Zehner, K. S., Johnson, J., \& Hirt, E. R. (1983). Social explanation: The role of timing, set, and recall on subjective likelihood estimates. Joumal of Personality and Social Psychology, 44, 1127-1143.

Showers, C. (1992). The motivational and emotional consequences of considering positive or negative possibilities for an upcoming event. Joumal of Personality and Social Psychology, 63, 474-484.

Steiner, G. (1975). After Babel: Aspects of language and translation. New York: Oxford University Press.

Taylor, S. E., \& Brown, J. D. (1988). Illusion and well-being: A social psychological perspective on mental health. Psychological Bulletin, 103, 193210.

Taylor, S. E., Buunk, B. P., \& Aspinwall, L. G. (1990). Social comparison, stress, and coping. Personality and Social Psychology Bulletin, 16, 74-89.

Taylor, S. E., \& Lobel, M. (1989). Social comparison activity under threat: Downward evaluation and upward contacts. Psychological Review, 96, 569575.

Taylor, S. E., \& Schneider, S. K. (1989). Coping and the simulation of events. Social Cognition, 7, 174-194.

Taylor, S. E., Wood, J. V., \& Lichtman, R. R. (1983). It could be worse: Selective evaluation as a response to victimization. Joumal of Social Issues, 39(2), 19-40.

Tversky, A., \& Kahneman, D. (1973). Availability: A heuristic for judging frequency and probability. Cognitive Psychology, 5, 207-232. 
Weiner, B. (1985). An attributional theory of achievement motivation and emotion. Psychological Review, 92, 548-573.

Wells, G. L., \& Gavanski, I. (1989). Mental simulation of causality. Joumal of Personality and Social Psychology, 56, 161-169.

Wells, G. L., Taylor, B. R., \& Turtle, J. W. (1987). The undoing of scenarios. Joumal of Personality and Social Psychology, 53, 421-430.

Wills, T. A. (1981). Downward comparison principles in social psychology. Psychological Bulletin, 90, 245-271.

Wood, J. V., Saltzberg, J. A., Neale, J. M., Stone, A. A., \& Rachmiel, T. B. (1990). Self-focused attention, coping responses, and distressed mood in everyday life. Joumal of Personaliny and Social Psychology, 58, 1027-1036.

Wood, J. V., Taylor, S. E., \& Lichtman, R. R. (1985). Social comparison in adjustment to breast cancer. Joumal of Personality and Social Psychology, 49. 1169-1183. 


\section{APPENDIX A}

\section{Examples of Counterfactuals}


Upward/Additive Counterfactuals

"If only I had confronted her from the onset of us not speaking, it would have made things easier in the end." (Experiment 1).

"If only I could have studied harder in the previous years of secondary school, I may not have encountered such difficulty in studying." (Experiment 2).

"I could have tried saying the words out loud until I recognized them." (Experiment 3).

\section{Upward/Subtractive Counterfactuals}

"I shouldn't have rushed into the relationship and become so attached in so little time." (Experiment 1).

"If only I hadn't spent so much time on the harmonization question." (Experiment 2).

"I should not have assumed that a consonant would start each word." (Experiment 3).

\section{Downward/Additive Counterfactuals}

"I might have kept my feelings to myself and it would have ate away at our friendship." (Experiment 1).

"If I had given up during the exam (even then I knew I would do poorly), it would have been worse." (Experiment 2).

"I could have chosen people and places as a topic and been confronted with words that were unfamiliar to me." (Experiment 3).

\section{Downward/Subtractive Counterfactuals}

"If my best friend hadn't approached me first after the fight, my stubborness wouldn't have allowed me to talk to her or apologize." (Experiment 1).

"But if I didn't spend those hours studying that I did, I would have done even worse in the exam." (Experiment 2).

"If I hadn't skipped one of the words, I would have lost a lot more time guessing, as I was having a lot of trouble visualizing it." (Experiment 3). 


\section{APPENDIX B}

\section{Materials - Experiment 1}




\section{Consent to Participate in Research}

Neal Roese and Dr. James Olson of the Department of Psychology are undertaking a study and request your participation. The study will involve completing questionnaires and will take less than an hour of your time. Participation in the experiment will not involve you in any known risks. Following the experiment, you will be provided with a complete explanation of the purposes and expected results of the study. All data gathered in this experiment will be confidential and for research purposes only. If you have any questions during the study, feel free to ask the experimenter.

As is the practice of this Department, you are free to withdraw from this study at any time for any reason without loss of experimental credit. 
This study is part of an ongoing examination of the processes underlying coping with negative life events.

Please take a moment to think of a single event in the last year that happened to you that was especially negative and/or diappointing. It should also directly involve at least one other person of your approximate age (for example, a fight between you and a friend).

In just a few words, please describe this event (keep in mind that this questionnaire is anonymous and confidential). 
People often have thoughts like "if only ..." after these sorts of negative events, in that they can see how things might have turned out better. For example, a Toronto woman who recently sustained minor injuries when she was hit by a car told reporters, "If only I had looked down that street a second time. I would've been fine." Often, we wish we had done something to avoid a negative outcome. In the space below, please list some specific actions that, in retrospect, could have been taken to have avoided the event you recalled. 
People often have thoughts like "if only ..." after these sorts of negative events. in that they can see how things might have turned out better. For example, a Toronto woman who recently sustained minor injuries when she was hit by a car told reporters. "If only I had NOT been in such a rush, I would've been fine." Often, we wish we hadn't done something that led to a negative outcome. In the space below, please list some specific actions that, in retrospect, should not have been done, which might have avoided the event you recalled. 
People often have thoughts like "well, at least ..." after these sorts of negative events, in that they can see how things might have turned out even worse. For example, a Toronto woman who recently sustained minor injuries when she was hit by a car told reporters, "At least no one moved me around after the accident, or it would've been a lot worse." Often, there are things that could have happened that would have made a negative outcome worse. In the space below, please list some specific actions that could have happened that would have made the event you recalled even worse. 
People often have thoughts like "well, at least ..." after these sorts of negative events, in that they can see how things might have turned out even worse. For example, a Toronto woman who recently sustained minor injuries when she was hit by car told reporters, "At least someone called an ambulance right away, or it would've been a lot worse." Often, negative outcomes could have been worse if certain actions had not been taken. In the space below, please list some specific actions that did occur in the event you recalled, and that would have made the event even worse if they had not occurred. 
Please indicate how thinking about this event makes you feel right now by circling a number on each of the following scales.

$\begin{array}{lllllllllll}\text { DEPRESSED } & -4 & -3 & -2 & -1 & 0 & +1 & +2 & +3 & +4 & \text { ELATED }\end{array}$

$\begin{array}{lllllllllll}\text { NEGATIVE } & -4 & -3 & -2 & -1 & 0 & +1 & +2 & +3 & +4 & \text { POSITIVE }\end{array}$

$\begin{array}{lllllllllll}\text { ANXIOUS } & -4 & -3 & -2 & -1 & 0 & +1 & +2 & +3 & +4 & \text { CALM } \\ \text { UNHAPPY } & -4 & -3 & -2 & -1 & 0 & +1 & +2 & +3 & +4 & \text { HAPPY } \\ & & & & & & & & & & \\ \text { HOSTILE } & -4 & -3 & -2 & -1 & 0 & +1 & +2 & +3 & +4 & \text { AGREEABLE }\end{array}$

$\begin{array}{llllllllllll}\text { DISAPPOINTED } & -4 & -3 & -2 & -1 & 0 & +1 & +2 & +3 & +4 & \text { RELIEVED }\end{array}$ 
Please circle the number that best represents your current perceptions of this event.

1. How responsible were you for this negative event?

$\begin{array}{lllllllllll}\text { NOT AT ALL } & -4 & -3 & -2 & -1 & 0 & +1 & +2 & +3 & +4 & \begin{array}{l}\text { COMPLETELY } \\ \text { RESPONSIBLE }\end{array} \\ \text { RESPONSIBLE } & & & & & & & & & \end{array}$

2. How responsible were other people for this negative event?

$\begin{array}{lllllllllll}\text { NOT AT ALL } & -4 & -3 & -2 & -1 & 0 & +1 & +2 & +3 & +4 & \begin{array}{l}\text { COMPLETELY } \\ \text { RESPONSIBLE }\end{array} \\ \text { RESPONSIBLE } & & & & & & & & & & \end{array}$

3. How much control did you have over this event?

NOT AT ALL

$\begin{array}{llllllllll}\text { UNDER MY } & -4 & -3 & -2 & -1 & 0 & +1 & +2 & +3 & +4\end{array}$

COMPLETELY CONTROL

$\begin{array}{llllllllll}-4 & -3 & -2 & -1 & 0 & +1 & +2 & +3 & +4 & \begin{array}{l}\text { COMPLETELY } \\ \text { UNDER MY } \\ \text { CONTROL }\end{array}\end{array}$

4. How avoidable was this event?

$\begin{array}{lllllllllll}\text { NOT AT ALL } & -4 & -3 & -2 & -1 & 0 & +1 & +2 & +3 & +4 & \text { COMPLETELY }\end{array}$ AVOIDABLE

AVOIDABLE 
Please take a moment to think of a single event in the last year that happened to you and that was especially positive and pleasant. It should also directly involve at least one other person of your approximate age (for example, you and a friend celebrated something together).

In just a few words, please describe this event (keep in mind that this questionnaire is anonymous and confidential). 
Please indicate how thinking about this second event makes you feel right now by circling a number on each of the following scales.

$\begin{array}{lllllllllll}\text { DEPRESSED } & -4 & -3 & -2 & -1 & 0 & +1 & +2 & +3 & +4 & \text { ELATED }\end{array}$

$\begin{array}{lllllllllll}\text { NEGATIVE } & -4 & -3 & -2 & -1 & 0 & +1 & +2 & +3 & +4 & \text { POSITIVE }\end{array}$

$\begin{array}{lccccccccccc}\text { ANXIOUS } & -4 & -3 & -2 & -1 & 0 & +1 & +2 & +3 & +4 & \text { CALM } \\ & & & & & & & & & & & \\ \text { UNHAPPY } & -4 & -3 & -2 & -1 & 0 & +1 & +2 & +3 & +4 & \text { HAPPY } \\ & & & & & & & & & & & \\ \text { HOSTILE } & -4 & -3 & -2 & -1 & 0 & +1 & +2 & +3 & +4 & \text { AGREEABLE }\end{array}$

$\begin{array}{lllllllllll}\text { DISAPPOINTED } & -4 & -3 & -2 & -1 & 0 & +1 & +2 & +3 & +4 & \text { RELIEVED }\end{array}$ 


\section{Attitude Scale \#1}

1. I am not likely to speak to people until they have spoken to me.

2. I feel confident of my appearance.

3. I would describe myself as self-confident.

4. I am a good mixer.

5. When in a group of people, I have trouble thinking of the right things to say.

6. I would describe myself as one who tries to master situations.

7. Other people look up to me.

8. When in a group of people, I usually do what the others want rather than make suggestions.

9. I enjoy social gatherings just to be with people.

10. When I am in disagreement with other people, my opinion usually prevails.

11. I would rather not have much responsibility for other people.

12. I cannot seem to get others to notice me.

13. I make a point of looking other people in the eye.

14. I feel comfortable being approached by someone in a position of authority.

15. I would describs myself as indecisive.

16. I have no doubt about my social competence. 


\section{Attitude Scale $1-$ Answer sheet}

not at all

character-

istic of me very much

character-

istic of me

\begin{tabular}{|c|c|c|c|c|c|c|c|c|}
\hline 1. 0 & 1 & 2 & 3 & $\overline{4}$ & 5 & 6 & 7 & $\overline{8}$ \\
\hline 2.0 & 1 & 2 & 3 & 4 & 5 & 6 & 7 & $\overline{8}$ \\
\hline 0 & 1 & 2 & 3 & 4 & 5 & 6 & 7 & $\overline{8}$ \\
\hline 0 & 1 & 2 & 3 & 4 & 5 & 6 & 7 & $\overline{8}$ \\
\hline 5. 0 & 1 & 2 & 3 & 4 & 5 & 6 & 7 & $\overline{8}$ \\
\hline 6.0 & 1 & 2 & 3 & 4 & 5 & $\overline{6}$ & 7 & $\overline{8}$ \\
\hline 7.0 & 1 & 2 & 3 & 4 & 5 & 6 & 7 & $\overline{8}$ \\
\hline 8.0 & 1 & 2 & 3 & 4 & 5 & 6 & 7 & $\overline{8}$ \\
\hline 9.0 & 1 & 2 & 3 & 4 & 5 & 6 & 7 & $\overline{8}$ \\
\hline 10.0 & 1 & 2 & 3 & 4 & 5 & 6 & 7 & 8 \\
\hline 11.0 & 1 & 2 & 3 & 4 & 5 & 6 & 7 & $\overline{8}$ \\
\hline$\overline{12 \cdot 0}$ & $\overline{1}$ & 2 & 3 & 4 & 5 & 6 & 7 & $\overline{8}$ \\
\hline 13.0 & 1 & 2 & 3 & 4 & 5 & 6 & 7 & $\overline{8}$ \\
\hline $14 \cdot 0$ & 1 & 2 & 3 & 4 & 5 & 6 & 7 & $\overline{8}$ \\
\hline 15.0 & 1 & 2 & 3 & 4 & 5 & 6 & 7 & $\overline{8}$ \\
\hline 16.0 & 1 & 2 & 3 & 4 & 5 & 6 & 7 & $\overline{8}$ \\
\hline
\end{tabular}


This experiment examined some very basic thought processes, namely, how people generate "if only" ideas. These are called counterfactuals (literally, contrary to the facts). For example. "If only I had brought my umbrella. I wouldn't have gotten my hair wet," is an alternate construction of reality, with just one element changed (bringing an umbrella). Psychologists are interested in these types of thoughts because they are closely linked to emotions, particularly when they follow negative events. Understanding how and why people generate counterfactuals allows us to better understand how people react to and cope with various negative occurrences, from simply getting one's hair wet to more serious problems such as trauma, sudden loss, and bereavement.

This was an exploratory study examining whether counterfactuals of different types have different consequences for both feelings and expectations for the future. You were asked to recall one negative event, and then to generate some counterfactual versions of it. Half of all subjects generated upward counterfactuals (if only things had turned out better) and the other half generated downward counterfactuals (at least things weren't worse). One hypothesis is that downward counterfactuals will result in relief and other more positive feelings. A second hypothesis is that upward counterfactuals heighten our expectations that we can avoid the future recurrence of a negative event. perhaps by forming plans for future behaviour.

Also, half of all subjects generated additive counterfactuals (those that add new details) and the other half generated subtractive counterfactuals (those that remove things that did happen). Another hypothesis is that additive combined with upward counterfactuals might result in especially negative feelings (I could have done something ... ).

These findings, if they occur, will be an important addition to our understanding of how counterfactual thoughts guide emotions and future actions. Such research may have important implications for our understanding of bereavement and coping.

Please be assured that all of your responses in this experiment will be treated as confidential. Your name will not be recorded or associated with your answers. Thus, your participation is completely anonymous.

If you are interested in counterfactual thinking and some of the ideas behind this study, you could read the articles listed below. You may also feel free to drop by either of our offices to discuss it.

Sincerely,

Neal Roese, M.A.

Doctoral Candidate in Psychology

Room 2254, Social Science Centre
James Olson. Ph.D.

Professor of Psychology

Room 6328. Social Science Centre

Phone: 679-2111. ext. 4657

\section{For Further Reading}

Miller, D. T., \& McFarland, C. (1986). Counterfactual thinking and victim compensation: A test of norm theory. Personality and Social Psychology Bulletin, 12, 513-519.

Miller, D. T., Turnbull, W., \& McFarland, C. (1990). Counterfactual thinking and social perception: Thinking about what might have been. In M. P. Zanna (Ed.), Advances in experimental social psychology (Vol. 23). New York: Academic Press. 


\section{APPENDIX C}

\section{Materials - Experiment 2}




\section{Consent to Participate in Research}

Neal Roese and Dr. James Olson of the Department of Psychology are undertaking a study and request your participation. The study will involve completing questionnaires and will take less than an hour of your time. Participation in the experiment will not involve you in any known risks. Following the experiment, you will be provided with a complete explanation of the purposes and expected results of the study. All data gathered in this experiment will be confidential and for research purposes only. If you have any questions during the study, feel free to ask the experimenter.

As is the practice of this Department, you are free to withdraw from this study at any time for any reason without loss of experimental credit. 
This study is part of an ongoing examination of the processes underlying coping with negative life events.

Please take a moment to think of an exam you wrote recently (e.g., in the last year) that went especially poorly, and that you were especially disappointed with.

In just a few words, please provide a few details about the exam (e.g., the course it was from, when you wrote it, etc.).

On the next few pages are several questions about your perceptions of your exam performance. Please answer them as completely as possible. 
People often have thoughts like "if only ... " after negative events, in that they can see how things might have turned out better. For example, a Toronto woman who recently sustained minor injuries when she was hit by a car told reporters, "If only I had looked down that street a second time, I would've been fine." Often, we wish we had done something to avoid a negative outcome. In the space below, please list some specific actions that, in retrospect, could have been taken to have improved your exam score. 
People often have thoughts like "if only ... " after negative events, in that they can see how things might have turned out better. For example, a Toronto woman who recently sustained minor injuries when she was hit by a car told reporters, "If only I had NOT been in such a rush. I would've been fine." Often, we wish we hadn't done something that led to a negative outcome. In the space below, please list some specific actions that. in retrospect. should not have been done, which might have improved your exam score. 
People often have thoughts like "well, at least ... " after negative events, in that they can see how things might have turned out even worse. For example, a Toronto woman who recently sustained minor injuries when she was hit by a car told reporters, "At least I didn't try to move around after the accident, or it would've been a lot worse." Often, there are things that could have happened that would have made a negative outcome worse. In the space below, please list some specific actions that you could have done that would have made your exam score even worse. 
People often have thoughts like "well, at least ... " after negative events, in that they can see how things might have turned out even worse. For example, a Toronto woman who recently sustained minor injuries when she was hit by car told reporters, "At least I had my medic-alert bracelet on, or it would've been a lot worse." Often, negative outcomes could have been worse if certain actions had not been taken. In the space below, please list some specific actions that you did before the exam, and that would have made your exam score even worse if you had not done them. 
Please indicate how thinking about your exam score makes you feel right now by circling a number on each of the following scales.

$\begin{array}{llllllllllll}\text { DISAPPOINTED } & -4 & -3 & -2 & -1 & 0 & +1 & +2 & +3 & +4 & \text { RELIEVED }\end{array}$

$\begin{array}{llllllllll}\text { CONCERNED } & -4 & -3 & -2 & -1 & 0 & +1 & +2 & +3 & +4 \\ \text { UNCONCERNED }\end{array}$

$\begin{array}{lllllllllll}\text { DEPRESSED } & -4 & -3 & -2 & -1 & 0 & +1 & +2 & +3 & +4 & \text { ELATED }\end{array}$

$\begin{array}{lllllllllll}\text { TIRED } & -4 & -3 & -2 & -1 & 0 & +1 & +2 & +3 & +4 & \text { AWAKE }\end{array}$

$\begin{array}{lllllllllll}\text { NEGATIVE } & -4 & -3 & -2 & -1 & 0 & +1 & +2 & +3 & +4 & \text { POSITIVE }\end{array}$

$\begin{array}{lllllllllll}\text { ANXIOUS } & -4 & -3 & -2 & -1 & 0 & +1 & +2 & +3 & +4 & \text { CALM }\end{array}$

$\begin{array}{llllllllllll}\text { UNHAPPY } & -4 & -3 & -2 & -1 & 0 & +1 & +2 & +3 & +4 & \text { HAPPY }\end{array}$

$\begin{array}{lllllllllll}\text { HOSTILE } & -4 & -3 & -2 & -1 & 0 & +1 & +2 & +3 & +4 & \text { AGREEABLE }\end{array}$ 
Please circle the number that best represents your current perceptions of this event.

1. How responsible were you for your exam performance!

$\begin{array}{lllllllllll}\text { NOT AT ALL } & -4 & -3 & -2 & -1 & 0 & +1 & +2 & +3 & +4 & \text { COMPLETELY }\end{array}$ RESPONSIBLE RESPONSIBLE

2. How responsible were other people for your exam performance?

$\begin{array}{lllllllllll}\text { NOT AT ALL } & -4 & -3 & -2 & -1 & 0 & +1 & +2 & +3 & +4 & \text { COMPLETELY }\end{array}$ RESPONSIBLE

RESPONSIBLE

3. How much control over your exam score did you have?

NOT AT ALL

$\begin{array}{llllllllll}\text { UNDER MY } & -4 & -3 & -2 & -1 & 0 & +1 & +2 & +3 & +4\end{array}$

COMPLETELY CONTROL

$\begin{array}{llllllllll}-4 & -3 & -2 & -1 & 0 & +1 & +2 & +3 & +4 & \begin{array}{l}\text { COMPLETELY } \\ \text { UNDER MY } \\ \text { CONTROL }\end{array}\end{array}$

4. How avoidable was your poor exam score?

$\begin{array}{lllllllllll}\text { NOT AT ALL } & -4 & -3 & -2 & -1 & 0 & +1 & +2 & +3 & +4 & \text { COMPLETELY }\end{array}$ AVOIDABLE

AVOIDABLE 
A) What grade-point average do you expect to achieve this year (please give a percentage, e.g., 65\%).

B) This section is a pretest for futur? research. Below you will find a list of school-related strategies of behaviour. All of them were collected from first year Western students last spring. Please indicate the likelihood that YOU might perform each of the following during the rest of this school year.

1. Studying / reviewing your notes on three or more nights during the week.

NOT AT ALL

$\begin{array}{llllllllll}\text { LIKELY TO } & -4 & -3 & -2 & -1 & 0 & +1 & +2 & +3 & +4\end{array}$

EXTREMELY DO THIS

LIKELY

TO DO THIS

2. No drinking or partying for one week before exams.

NOT AT ALL

$\begin{array}{llllllllll}\text { LIKELY TO } & -4 & -3 & -2 & -1 & 0 & +1 & +2 & +3 & +4\end{array}$

EXTREMELY DO THIS

LIKELY

TO DO THIS

3. Regularly seeing your professors to ask questions and discuss problem areas.

NOT AT ALL

$\begin{array}{llllllllll}\text { LIKELY TO } & -4 & -3 & -2 & -1 & 0 & +1 & +2 & +3 & +4\end{array}$

EXTREMELY DO THIS

LIKELY

TO DO THIS

4. No watching TV on at least three nights during the week before exams.

NOT AT ALL

$\begin{array}{llllllllll}\text { LIKELY TO } & -4 & -3 & -2 & -1 & 0 & +1 & +2 & +3 & +4\end{array}$

EXTREMELY

DO THIS

LIKELY

TO DO THIS 
5. Trying to attend every lecture during the school year.

NOT AT ALL

$\begin{array}{llllllllll}\text { LIKELY TO } & -4 & -3 & -2 & -1 & 0 & +1 & +2 & +3 & +4\end{array}$ DO THIS

$\begin{array}{llllllllll}-4 & -3 & -2 & -1 & 0 & +1 & +2 & +3 & +4 & \begin{array}{l}\text { EXTREMELY } \\ \text { LIKELY } \\ \text { TO DO THIS }\end{array}\end{array}$

6. Not going away on the weekend before the week of an exam.

NOT AT ALL

$\begin{array}{llllllllll}\text { LIKELY TO } & -4 & -3 & -2 & -1 & 0 & +1 & +2 & +3 & +4\end{array}$ DO THIS

EXTREMELY

LIKELY

TO DO THIS 
Please take a moment to think of a single event in the last year that happened to you and that was especially positive and pleasant. It should also directly involve at least one other person of your approximate age (for example, you and a friend celebrated something together).

In just a few words, please describe this event (keep in mind that this questionnaire is anonymous and confidential). 
Please indicate how thinking about this second event makes you feel right now by circling a number on each of the following scales.

$\begin{array}{lllllllllll}\text { DEPRESSED } & -4 & -3 & -2 & -1 & 0 & +1 & +2 & +3 & +4 & \text { ELATED }\end{array}$

$\begin{array}{llllllllllll}\text { NEGATIVE } & -4 & -3 & -2 & -1 & 0 & +1 & +2 & +3 & +4 & \text { POSITIVE }\end{array}$

$\begin{array}{lllllllllll}\text { ANXIOUS } & -4 & -3 & -2 & -1 & 0 & +1 & +2 & +3 & +4 & \text { CALM }\end{array}$

$\begin{array}{lllllllllll}\text { UNHAPPY } & -4 & -3 & -2 & -1 & 0 & +1 & +2 & +3 & +4 & \text { HAPPY }\end{array}$

$\begin{array}{lllllllllll}\text { HOSTILE } & -4 & -3 & -2 & -1 & 0 & +1 & +2 & +3 & +4 & \text { AGREEABLE }\end{array}$

$\begin{array}{lllllllllll}\text { DISAPPOINTED } & -4 & -3 & -2 & -1 & 0 & +1 & +2 & +3 & +4 & \text { RELIEVED }\end{array}$ 


\section{Attitude Scale \#1}

1. I am not likely to speak to people until they have spoken to me.

2. I feel confident of my appearance.

3. I am sometimes irritated by people who ask favours of me.

4. I would describe myself as self-confident.

5. I sometimes try to get even, rather than forgive and forget.

6. I am good at meeting new people at social gatherings.

7. Sometimes at elections I vote for candidates I know little about.

8. When in a group of people, I have trouble thinking of the right things to say.

9. I would describe myself as one who tries to master situations.

10. I always apologize to others for my mistakes.

11. I would describe myself as having a good sense of humour.

12. Other people look up to me.

13. I always declare everything at the border, even when there is no chance that I could be found out.

14. When in a group of people, I usually do what the others want rather than make suggestions.

15. I am always courteous, even to people I find annoying.

16. I enjoy social gatherings just to be with people.

17. When I am in disagreement with other people, my opinion usually prevails.

18. I sometimes tell lies if I have to.

19. I would rather not have much responsibility for other people.

20. I cannot seem to get others to notice me. 
21. I make a point of looking other people in the eye.

22. I feel comfortable being approached by someone in a position of authority.

23. I would describe myself as indecisive.

24. I have no doubt about my social competence. 


\section{Attitude Scale 1 - Answer sheet}

not at all

very much

character-

character-

istic of me

\begin{tabular}{llllllllll}
\hline 1. & 0 & 1 & 2 & 3 & 4 & 5 & 6 & 7 & 8
\end{tabular}

\begin{tabular}{llllllllll}
\hline 2. & 0 & 1 & 2 & 3 & 4 & 5 & 6 & 7 & 8
\end{tabular}

\begin{tabular}{llllllllll}
\hline 3. & 0 & 1 & 2 & 3 & 4 & 5 & 6 & 7 & 8 \\
\hline
\end{tabular}

\begin{tabular}{llllllllll}
\hline 4. & 0 & 1 & 2 & 3 & 4 & 5 & 6 & 7 & 8 \\
\hline
\end{tabular}

\begin{tabular}{llllllllll}
\hline 5. & 0 & 1 & 2 & 3 & 4 & 5 & 6 & 7 & 8
\end{tabular}

\begin{tabular}{llllllllll}
\hline 6. & 0 & 1 & 2 & 3 & 4 & 5 & 6 & 7 & 8
\end{tabular}

\begin{tabular}{llllllllll}
\hline 7. & 0 & 1 & 2 & 3 & 4 & 5 & 6 & 7 & 8
\end{tabular}

\begin{tabular}{llllllllll}
\hline 8. & 0 & 1 & 2 & 3 & 4 & 5 & 6 & 7 & 8
\end{tabular}

\begin{tabular}{llllllllll}
\hline 9. & 0 & 1 & 2 & 3 & 4 & 5 & 6 & 7 & 8
\end{tabular}

\begin{tabular}{lllllllll}
\hline 10. & 0 & 1 & 2 & 3 & 4 & 5 & 6 & 7 \\
\hline
\end{tabular}

\begin{tabular}{lllllllll}
\hline 11. & 0 & 1 & 2 & 3 & 4 & 5 & 6 & 7 \\
\hline
\end{tabular}

\begin{tabular}{llllllllll}
\hline 12. & 0 & 1 & 2 & 3 & 4 & 5 & 6 & 7 & 8
\end{tabular}

\begin{tabular}{llllllllll}
\hline 13. & 0 & 1 & 2 & 3 & 4 & 5 & 6 & 7 & 8
\end{tabular}

\begin{tabular}{llllllllll}
\hline 14.0 & 1 & 2 & 3 & 4 & 5 & 6 & 7 & 8
\end{tabular}

\begin{tabular}{llllllllll}
\hline 15. & 0 & 1 & 2 & 3 & 4 & 5 & 6 & 7 & 8
\end{tabular}

\begin{tabular}{llllllllll}
\hline 16. & 0 & 1 & 2 & 3 & 4 & 5 & 6 & 7 & 8
\end{tabular}

\begin{tabular}{lllllllll}
\hline 17. & 0 & 1 & 2 & 3 & 4 & 5 & 6 & 7
\end{tabular}

\begin{tabular}{llllllllll}
\hline 18. & 0 & 1 & 2 & 3 & 4 & 5 & 6 & 7 & 8
\end{tabular}

\begin{tabular}{llllllllll}
\hline 19. & 0 & 1 & 2 & 3 & 4 & 5 & 6 & 7 & 8
\end{tabular}

\begin{tabular}{llllllllll}
\hline 20. & 0 & 1 & 2 & 3 & 4 & 5 & 6 & 7 & 8
\end{tabular}

\begin{tabular}{llllllllll}
\hline 21. & 0 & 1 & 2 & 3 & 4 & 5 & 6 & 7 & 8
\end{tabular}

\begin{tabular}{llllllllll}
\hline 22. & 0 & 1 & 2 & 3 & 4 & 5 & 6 & 7 & 8 \\
\hline
\end{tabular}




\begin{tabular}{llllllllll}
\hline $23 \cdot$ & 0 & 1 & 2 & 3 & 4 & 5 & 6 & 7 & 8 \\
\hline $24 \cdot 0$ & 1 & 2 & 3 & 4 & 5 & 6 & 7 & 8 \\
\hline
\end{tabular}




\section{Post-Experimental Questionnaire}

1. Were there any parts of this study that were unclear, or were worded in a way that was difficult to follow?

2. What did you think the main point of this study was?

3. What sorts of results do you expect we will find?

4. Earlier in this study, you recorded "if only" or "what if" thoughts of how things could have turned out differently. How do you think these thoughts are related to emotions?

How do you think these thoughts are related to expectations for the future? 
This experiment examined some very basic thought processes, namely, how people generate "if only" ideas. These are called counterfactuals (literally, contrary to the facts). For example, "If only I had brought my umbrella. I wouldn't have gotten my hair we:." is an alternate construction of reality. with just one element changed (bringing an umbrella). Psychologists are interested in these types of thoughts because they are clusely linked to emotions, particularly when they follow negative events. Understanding how and why people generate counterfactuals allows us to better understand how people react to and cope with various negative occurrences, from simply getting one's hair wet to more serious problems such as trauma, sudden loss, and bereavement.

This was an exploratory study examining whether counterfactuats of differen: ypes have different consequences for both feeings and expectations for te luture. You were asked to recall ore negative event (a poor exan peiformance), and .en to generate some counterfactual versions of it. Half of all subjects generated upward wunterfactuals (if only things had turned out better) and the other half generated downward counterfaciuals (at least things werent worse). One hypothesis is that downward ceunterfactuals will result in relief and other more positive feelings. A second hypothesis is that upward counterfactuais heighten our expectations that we can avoid the future recurrence of a negative event, perhaps by forming plans for future behaviour.

Also, half of all subjects generated "additive" counterfactuals (those that add new details $i$ and the other half generated "subtractive" counterfactuals (those that remove things that did happen). Another hypothesis is that additive counterlactuals also can heighten expectations for improvement.

These findings, if they occur, will be an important addition to our understanding of hou counterl cetual thoughts guide emotions and futuie actions. Such research may have important implications for our understanding of bereavement and coping.

Please be assured that all of your responses in this experiment will be treated as confidential. Your name will not be recorded or associated with your answers. Thius. your participation is completely anonymous.

If you want to learn more about counterfactual thinking and some of the ideas hehind this : :udy, you could read the articles listed betow. You may also feel free to drop by cither o! our oftices to riscuss it.

Sin_crely.

Vial Recise. M.A.

Doctorai Candiatate in Pisychology

$R(x) m$ 225: Socidal Si ience Centre

James Olson, Ph.D.

Professor of Psychology

Room 6.328, Sccial Science Centre

Phone: 679.2111. ext. 46.57

\section{For Further Reading}

Milier. D. T., \& McFariand. C. (1980). Counterfactual thinking and victim compens: tien: A lest of norin theory. Personality and Social Psychologr Bulletin, 12, 513-519.

Miller. D. T. Turnhull. W.. \& McFarland. C. (196()). Counterfactual thinking and sociod perceptus: Thinking about what might have been. In M. P. Zanna (Ed.). Adrances in exiermental suic: psicholage (Vol. 23). New York: Academic Press.

Row.e. N. J. \& Olson. J. M. (in press). The :tructure of counterfactual thought.

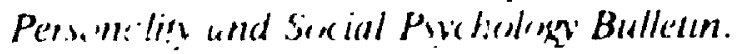




\section{APPENDIX D}

Materials - Experiment 3 


\section{Consent to Participate in Research}

Neal Roese and Dr. James Olson of the Department of Psychology are undertaking a study and request your participation. The study will involve completing a computer-administered anagram-solving task, and filling out a questionnaire that asks about the computer task. It will take less than an hour of your time and is worth one experimental credit. Participation in the experiment will not involve you in any known risks. Following the experiment, you will be provided with a complete explanation of the purposes and expected results of the study. All data gathered in this experiment will be confidential and for research purposes only. If you have any questions during the study, feel free to ask the experimenter.

As is the practice of this Department, you are free to withdran' from this study at any time for any reason without loss of experimental credit. 
People often evaluate their past performance with thoughts like "if only ... ". These thoughts focus on how things might have turned out better. For example, a Toronto woman who recently sustained minor injuries when she was hit by a car told reporters, "If only I had looked down that street a second time, I would've been fine." Often, we wish we had done something to avoid a certain outcome. In the space below, please list any specific actions that you could have taken to improve your anagram score. 
People often evaluate their past performance with thoughts like "if only ... ". These thoughts focus on how things might have turned out better. For example, a Toronto woman who recently sustained minor injuries when she was hit by a car told reporters, "If only I had NOT been in such a rush, I would've been fine." Often, we wish we hadn't done something that led to a certain outcome. In the space below, please list any specific actions that you should not have taken to improve your anagram score. 
People often evaluate their past performance with thoughts like "well, at least ... ". These thoughts focus on how things might have turned out even worse. For example, a Toronto woman who recently sustained minor injuries when she was hit by a car told reporters, "At least I didn't try to move around after the accident, or it would've been a lot worse." Often, there are things that could have happened that would have made a given outcome worse. In the space below, please list any specific actions that you could have taken to make your anagram score worse. 
People often evaluate their past performance with thoughts like "well, at least ... ". These thoughts focus on how things might have turned out even worse. For example, a Toronto woman who recently sustained minor injuries when she was hit by car told reporters, "At least I had my medic-alert bracelet on, or it would've been a lot worse." Often, negative outcomes could have been worse if certain actions had not been taken. In the space below, please list any specific actions that you believe enhanced your anagram score, and that would make your anagram score worse if you hadn't performed them. 
Please indicate your reaction to your anagram score by circling a number on each of the following scales.

$\begin{array}{llllllllllll}\text { DISAPI JINTED } & -4 & -3 & -2 & -1 & 0 & +1 & +2 & +3 & +4 & \text { RELIEVED }\end{array}$

$\begin{array}{llllllllll}\text { CONCERNED } & -4 & -3 & -2 & -1 & 0 & +1 & +2 & +3 & +4 \\ \text { UNCONCERNED }\end{array}$

$\begin{array}{lllllllllll}\text { DEPRESSED } & -4 & -3 & -2 & -1 & 0 & +1 & +2 & +3 & +4 & \text { ELATED }\end{array}$

TIRED

$\begin{array}{llllllllll}-4 & -3 & -2 & -1 & 0 & +1 & +2 & +3 & +4 & \text { AWAKE }\end{array}$

$\begin{array}{lllllllllll}\text { NEGATIVE } & -4 & -3 & -2 & -1 & 0 & +1 & +2 & +3 & +4 & \text { POSITIVE }\end{array}$

$\begin{array}{llllllllllll}\text { ANXIOUS } & -4 & -3 & -2 & -1 & 0 & +1 & +2 & +3 & +4 & \text { CALM }\end{array}$

$\begin{array}{lllllllllll}\text { UNHAPPY } & -4 & -3 & -2 & -1 & 0 & +1 & +2 & +3 & +4 & \text { HAPPY }\end{array}$

$\begin{array}{lllllllllll}\text { HOSTILE } & -4 & -3 & -2 & -1 & 0 & +1 & +2 & +3 & +4 & \text { AGREEABLE }\end{array}$ 
Please circle the number that best represents your current perceptions of your performance on the anagram task.

1. How responsible were you for your anagram score?

$\begin{array}{lllllllllll}\text { NOT AT ALL } & -4 & -3 & -2 & -1 & 0 & +1 & +2 & +3 & +4 & \text { COMPLETELY }\end{array}$ RESPONSIBLE RESPONSIBLE

2. How difficult did your anagrams seem to you?

$\begin{array}{lllllllllll}\text { NOT AT ALL } & -4 & -3 & -2 & -1 & 0 & +1 & +2 & +3 & +4 & \text { EXTREMELY }\end{array}$ DIFFICULT

DIFFICULT

3. How much control over your anagram score did you have?

NOT AT ALL

$\begin{array}{llllllllll}\text { UNDER MY } & -4 & -3 & -2 & -1 & 0 & +1 & +2 & +3 & +4\end{array}$

COMPLETELY CONTROL

4. How avoidable was your anagram score?

$\begin{array}{lllllllllll}\text { NOT AT ALL } & -4 & -3 & -2 & -1 & 0 & +1 & +2 & +3 & +4 & \text { COMPLETELY }\end{array}$ AVOIDABLE 
In just a few moments, you will attempt to solve a second set of ten anagrams, presented to you on the computer in exactly the same way.

5. How do you expect to perform on this next set of anagrams? (circle the number that is most appropriate).

$(0)$

$(+1)$

much

a little

about

a little

much

worse

bit worse

the same

bit better

better 


\section{Post-Experimental Questionnaire}

Several questions are presented below that ask you about your own perceptions of this study. These responses will help us to improve future studies. Please answer them as honestly as possible. but also feel free to skip over any that you don't want to answer.

1. Were there any parts of this study that were unclear, or were worded $i_{i}$ a way that was difficult to follow?

2. What did you think the $m$ in point of this study was?

3. What sorts of resul's do you expect we will find?

4. Earlier in this study, you recorded "if only" or "what if" thoughts of how things could have turned out differently. How do you think these thoughts are related to emotions?

How do you think these thoughts are related to expectations for the future? 
This experiment examined some very basic thought processes, namely, how people generate "if only" ideas. These are called counterfactuals (literally, contrary to the facts). For example, "If only I had brought my umbrella, I wouldn't have gotten my hair wet," is an alternate construction of reality, with just one element changed (bringing an umbrella). Psychologists are interested in these types of thoughts because they are closely linked to emotions, particularly when they follow negative events. Understanding how and why people generate counterfactuals allows us to better understand how people react to and cope with various negative occurrences, from simply getting one's hair wet to more serious problems such as trauma, sudden loss, and hereavement.

This study examined whether counterfactuals of different types have different consequences for feelings, expectations, and future performance. You attempted to solve ten anagrams, and then were presented with feedback on your performance. Although your score accurately reflected how you did, you were also presented with false feedback about how others performed. Regardless of they actually performed, all participants were told that their score was 50 points below the average of all participants. Thus, even if your performance on the anagram task was quite good, you were automatically told that you ranked 37th out of 45 . This was an essential part of the experiment, for two reasons: 1) to motivate you to generate MORE counterfactual thoughts, and 2) to to motivate you to try to do better on the second anagram task.

After the first anagram set, participants generated counterfactuals. Half of all subjects generated "upward" counterfactuals (if only things had turned out better) and the other half generated "downward" counterfactuals (at least things weren't worse). One hypothesis is that downward counterfactuals will result in relief and other more positive feelings. A suconrt hypothesis is that upward counterfactuals heighten our expectations that we can avoid the future recurrence of a negative event, perhaps by forming plans for future behaviour. By examining your performance on the second anagram set, we can test this idea directly.

Also, half of all subjects generated "additive" counterfactuals (those that add new details) and the other half generated "subtractive" counterfactuals (those that remove things that did happen). Another hypothesis is that additive counterfactuals also can help us to improve our performance.

These findings. if they occur, will add to our knowledge of how counterfactual thoughts guide both our emotions and our future actions. Such research may also have implications for our understanding of bereavem: $\mathrm{nt}$ and coping.

"lease be assured that all of your responses in this experiment will be treated as confidential. You. name will not be recorded or associated with your answers. Thus, your participation is completely anonymous.

If you want to learn more about counterfactual thinking and some of the ldeas behind this study, you could read the articles listed below. You may also feel free to visit our offices to discuss it.

Sincerely, Neal Roese, M.A.

Doctoral Candidate in Psychology

Room 2254, Social Science Centre

James Olson. Ph.D.

Professor of Psychology

Room 6328, Social Science Centre

Phone: 679-2111, ext. 4657

\section{For Further Reading}

Miller, D. T., \& McFarland. C. (1986). Counterfactual thinking and victim compensation: A test of norm theory. Personality and Social Psychology Bulletin. 12, 513-519.

Miller. D. T., Turnbull, W., \& McFarland, C. (1990). Counterfactual thinking and social perception: Thinking about what might have been. In M. P. Zanna (Ed.). Adivances in experimental soc al psychology (Vol. 23). New York: Academic Press.

Rocse, N. J., \& Olson. J. M. (in press). The structure of counterfactual thought. Personality and Social Psychologv Bulletin. 


\section{APPENDIX E}

\section{BASIC Computer Program - Experiment 3}


'The Anagram Study.

- Effects of Counterfactual Structure and Direction on

' Affect, Expectancy, and Performance.

- Laboratory experiment created August 1992, (c) Neal Roese,

- University of Western Ontario

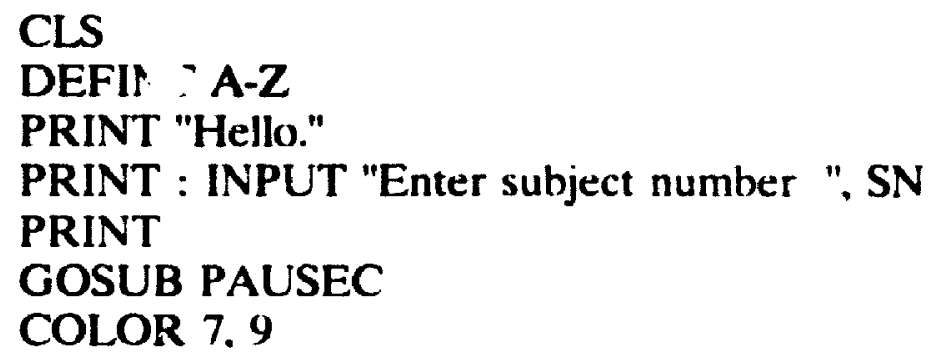

\section{CLS}

LOCATE 7, 26: PRINT "Human Decision-Making Study" GOSUB PAUSEC

1 CLS

INPUT "password: ", PWS

IF PWS $<>$ "sat" THEN GOTO 1

GOSUB SCRDEL:

START:

CLS

LOCATE 1, 28: PRINT "Human Decision-Making." LOCATE 3, 1

PRINT " This is a study of decision-making proceses under time presure."

PRINT "Your task will be to solve a series of anagrams within a certain period of $"$

PRINT "time. Anagrams are scrambled word combinations -- solving them simply means"

PRINT "unscrambling them into an actual word. For example. 'YHAPP' is an anagram and "

PRINT "its solution is 'HAPPY'. All the anagrams in this study have only ONE " PRINT "solution."

PRINT

PRINT " For each anagram solved, you will be awarded a certain number of " PRINT "points. At the end of the anagram task, the computer will calculate a" PRINT "total score, and then it will tell you how good this score is compared to" PRINT "other participants in this study." 


\section{CLS}

PRINT

PRINT " You will be awarded a maximum of 120 points for each anagram solved. "

PRINT "However, the longer it takes for you to solve the anagram, the lower your"

PRINT "score. One point will be deducted per second. So if you took 20 seconds"

PRINT "to solve an anagram, you would get 100 points; if you took 60 seconds to" PRINT "solve it, you would get only 60 points. You have a total of 2 minutes" PRINT "(120 seconds) to solve each anagram. After 2 minutes have elapsed, the" PRINT "computer will give you the solution and present the next anagram." PRINT "'!

PRINT " But tha's not all. If you cannot solve the anagram within the 2" PRINT "minute period, you will lose points. One point will be deducted from your"

PRINT "TOTAL score for each second spent on an anagram you didn't solve. This"

PRINT "means that it's a good idea to SKIP OVER anagrams that seem especially"

PRINT "difficult; this allows you to minimize the points lost. Therefore, deciding" PRINT "which anagrams to skip over is one of the most important decisions you'll"

PRINT "make in this task. You can skip ahead at any time simply by pressing $<$ S $>$ on"

PRINT "your keyboard."

GOSUB LOOPY

GOSUB PAUSEC

\section{CLS}

PRINT

PRINT " If you think you are near to solving :he anagram but can't quite get it,"

PRINT "you may 'buy' a clue. This will cost you 30 points from your score. The" PRINT "clue will be the middle letter of the correct solution. So for the anagram" PRINT "'YHAPP', you would get the clue 'P', which is the middle letter of the solution "

PRINT "'HAPPY'. You can request this clue at any time by simply pressing $\langle\mathrm{C}\rangle$ on your "

PRINT "keyboard."

PRINT

PRINT " When you figure out the solution to the anagram, press $<R>$ on your"

PRINT "keyboard, then type the five letters in, then press <ENTER>. The 
computer "

PRINT "will tell you immediately if you are right or wrong. "

PRINT

PRINT " Don't worry about remembering which letters to press. The computer will"

PRINT "remind you which ones you need to know."

GOSUB LOOPY

GOSUB PAUSEC

\section{CLS}

PRINT

PRINT " One last thing. A timer will be displayed onscreen to let you know how"

PRINT "much time you have left. When you type in your solution, the timer "

PRINT "display shuts off. However, YOUR TIME IS STILL RUNNING OUT! The clock inside"

PRINT "the computer will stop ONLY when a correct solution has been entered. So"

PRINT "it's a good idea to type in your solution (and then press the <ENTER> key) as"

PRINT "FAST AS YOU CAN."

GOSUB LOOPY

IF $($ HR = 1) THEN GOTO 355

GOSUB PAUSEC

GOSUB SCRDEL:

6 CLS

PRINT

PRINT " If you would need to read over these instructions a second time, press $"$

PRINT " $<G>$. If anything is unclear to you, please ask the experimenter now. " PRINT "Otheiwise, press $<\mathrm{C}>$ to continue."

\section{GOSUB LOOPY}

8 VS = INKEY\$

WHILE (VS = "'!): V\$ = INKEYS: WEND

IF (ASC(V\$) $=103$ ) THEN GOTO START

IF (ASC(V\$) $<>99$ ) THEN GOTO 8

\section{CLS}

PRINT : PRINT : PRINT : PRINT : PRINT

COLOR 7, 4

PRINT " Before you begin, you have the opportunity to select some of the " PRINT "parameters of the task. Specifically, you get to decide 1) how long you" PRINT "get to 'rest' between each anagram, 2) the subject area from which the" 
PRINT " anagrams are taken, and 3) the difficulty level of the anagrams. " COLOR 7,9

GOSUB LOOPY

GOSUB PAUSEC

12 CLS

PRINT

PRINT "1. How many seconds between each anagram?"

PRINT " (choose any number between $<1>$ and $<9>$ seconds, then press $<$ ENTER > )"

LOCATE 5,5

INPUT ${ }^{m !}, T$

IF $(\mathrm{T}<1)$ OR $(\mathrm{T}>9)$ THEN GOTO 12

PRINT

13 LOCATE 7, 1

PRINT "2. What topic do you prefer?"

PRINT " press $<1>$ for 'science and nature""

PRINT " press <2> for 'people and events"

LOCATE 11, 5

INPUT "'", ST

IF (ST $<>$ 1) AND (ST $<>$ 2) THEN GOTO 13

PRINT

15 LOCATE 13, 1

PRINT "3. What difficulty level would you like?"

PRINT " press $<1>$ for 'easy"

PRINT " press $<2>$ for 'challenging' ( 10 bonus points for each anagram solved)"

LOCATE 17, 5

INPUT '"', HH

IF $(\mathrm{HH}<>1)$ AND $(\mathrm{HH}<>2)$ THEN GOTO 15

GOSUB LOOPY

GOSUB PAUSEC

\section{CLS}

\section{PRINT}

PRINT " The anagram task will now begin. There will be no practice trial, so" PRINT "if you are unsure about anything, please ask the experimenter now.

PRINT "Otherwise, press <ENTER > to begin."

GOSUB LOOPY

DO UNTIL INKEYS = CHRS(13)

LOOP

20 CLS

INPUT "password: ", PWS 
IF PW\$ $<>$ "rain" THEN GOTO 20

MAIN:

CLS

TYPE STUDENT

ANA AS STRING * 5

CLUE AS STRING * 1

SOLUT AS STRING * 5

END TYPE

DIM S(1 TO 40) AS STUDENT

FOR I = 1 TO 10

READ S(I).ANA, S(I).CLUE, S(I).SOLUT

NEXT

IF ST $=2$ THEN GOTO 40

FOR I $=1$ TO 10

READ S(I).ANA, S(I).CLUE, S(I).SOLUT NEXT

' Main Loop, First Series

40 FOR $\mathrm{L}=1$ TO 10

GOSUB SCRDEL:

CLS

$\mathrm{CL}(\mathrm{L})=0 \quad$ 'initial setting of clue indicator

SK(L) $=0 \quad$ 'initial setting of skip indicator

LOCATE 10, 37: COLOR 7, 0

PRINT S(L).ANA

LOCATE 9, 31: PRINT "

LOCATE 11, 31: PRINT "

LOCATE 10, 31: PRINT "

LOCATE 10, 42: PRINT"

LOCATE 21, 1: COLOR 7, $4^{\text {' white on red }}$

PRINT" seconds

LOCATE 20, 1

PRINT "

LOCATE 22, 1

PRINT " remaining:

LOCATE 23, 1

PRINT "

LOCATE 15, 55: COLOR 0, 3

PRINT " Keys to press: 
LOCATE 16, 55

PRINT "

11

LOCATE 17, 55

PRINT " $<R>$ to enter solution "

LOCATE 18, 55

PRINT "

"

LOCATE 19, 55

PRINT " $<\mathrm{C}>$ to get clue

LOCATE 20, 55

PRINT " (costs 30 pts)

LOCATE 21, 55

PRINT "

LOCATE 22, 55

PRINT " $<S>$ to skip to next "

LOCATE 23, 55

PRINT " anagram

LOCATE 1, 1: COLOR 7, 0

PRINT "

LOCATE 2, 1

PRINT " Trial \#"

LOCATE 2, 10: PRINT USING "\#\#"; L

LOCATE 2, 12: PRINT " "

LOCATE 3, 1

PRINT "

'Main timer loop.

TIMES $=$ "00:00"

START $=$ TIMER

$100 \mathrm{AS}={ }^{\prime \prime \prime}$

WHILE (TIMER < 120) AND (AS = '"')

AS = INKEYS

LOCATE 22, 13: COLOR 7, 4

PRINT USING "\#\#\#\#.\#\# "; 120 - TIMER WEND

IF (TIMER > 119) THEN GOTO 200

'Time is out, goes to next trial.

IF $(\operatorname{ASC}(A S)=99)$ THEN GOSUB CLUE 'Subroutine for clue (c).

IF $(\operatorname{ASC}(A S)=115)$ THEN $\operatorname{SK}(L)=1$ 'skip indicator

IF $(A S C(A S)=115)$ THEN GOTO 200 'Subject skips to next trial (s).

LOCATE 15, 35: COLOR 7, 1: PRINT "

IF (ASC(A\$) $<114$ ) GOTO 100 
LOCATE 15, 37: COLOR 7, 1

INPUT '"', DS

IF DS $=S(L) . S O L U T$ THEN GOSUB CORRECT ELSE GOSUB WRONG

IF $D \$=S(L) . S O L U T$ THEN GOTO 250 ELSE GOTO 100

200 LOCATE 17, 31: COLOR 7, 0: PRINT" Solution:

LOCATE 18, 37:

'Prints solution at end of trial.

PRINT S(L).SOLUT

LOCATE 18, 31: PRINT" "

LOCATE 18, 42: PRINT" "

LOCATE 17, 29: COLOR 7, 9: PRINT " "

LOCATE 17, 48: PRINT "

250 LOCATE 21, 26: COLOR 14, 0

PRINT " Score for this trial: "

$\mathrm{SC}(\mathrm{L})=120-$ INT(TIMER)

IF CL(L) $=1$ THEN SC(L) $=$ SC $(L)-30$

IF SK(L) $=1$ THEN SC(L) $=$ INT(0 - TIMER)

IF CL(L) $=1$ AND SK(L) $=1$ THEN SC $(L)=($ INT $(0-$ TIMER $))-30$

LOCATE 21, 49: PRINT SC(L)

TIMES $=$ "00:00"

START = TIMER

WHILE (TIMER < 5)

WEND 'Program pauses for 5 secs.

IF $\mathrm{L}=10$ GOTO 300

COLOR 7,9

CLS

LOCATE 12, 33: PRINT "Get Ready ... "

TIMES = "00:00"

START = TIMER

WHILE (TIMER < T)

WEND

'Program pauses for $T$ secs.

300 GOSUB LOOPY

NEXT

$\mathrm{TOT}=\mathrm{SC}(1)+\mathrm{SC}(2)+\mathrm{SC}(3)+\mathrm{SC}(4)+\mathrm{SC}(5)+\mathrm{SC}(6)+\mathrm{SC}(7)+\mathrm{SC}(8)+$ $\mathrm{SC}(9)+\mathrm{SC}(10)$

$\mathrm{SK}=\mathrm{SK}(1)+\mathrm{SK}(2)+\mathrm{SK}(3)+\mathrm{SK}(4)+\mathrm{SK}(5)+\mathrm{SK}(6)+\mathrm{SK}(7)+\mathrm{SK}(8)+$ SK(9) + SK(10)

$\mathrm{CL}=\mathrm{CL}(1)+\mathrm{CL}(2)+\mathrm{CL}(3)+\mathrm{CL}(4)+\mathrm{CL}(5)+\mathrm{CL}(6)+\mathrm{CL}(7)+\mathrm{CL}(8)+$ $\mathrm{CL}(9)+\mathrm{CL}(10)$ 
$\mathrm{RA}=\mathrm{RA}(1)+\mathrm{RA}(2)+\mathrm{RA}(3)+\mathrm{RA}(4)+\mathrm{RA}(5)+\mathrm{RA}(6)+\mathrm{RA}(7)+\mathrm{RA}(8)$

$+\mathbf{R A}(9)+\mathbf{R A}(10)$

GOSUB SCRDEL:

CLS

COLOR 7, 1

PRINT

PRINT " This first task is now completed. Press <ENTER > to find out your" PRINT "total score."

GOSUB LOOPY

DO UNTIL INKEY\$ = CHR\$(13)

LOOP

GOSUB SCRDEL:

CLS

COLOR 7, 4

CLS

PRINT

PRINT " Participant: \#42"

PRINT

PRINT " Number of Correct"

PRINT " $\quad$ Solutions: $\quad$ "; RA; '"'

PRINT " Number of skips: "; SK; "'

PRINT " Number of clues: $\quad$ "; CL; "'"

PRINT

COLOR 7, 9

PRINT " Total Score:

LOCATE 9, 50: PRINT "

PRINT" Average score (of

PRINT " :ill participants): "; TOT + 53; "

LOCATE 11, 50: PRINT "

COLOR 7, 4

PRINT

PRINT " Your rank (out of all

PRINT " other participants): 37th out of 45 "

GOSUB LOOPY

GOSUB PALSEC

COLOR 7, 9

CLS

PRINT

PRINT " Please tell your experimenter that you are ready for the next task." 330 CLS

INPUT "password: ", PWS 
IF PW\$ $<>$ "boston" THEN GOTO 330

GOSUB SCRDEL:

'Second Phase

FOR $L=1$ TO $10 \quad$ 'tesets clue and right answer indicator

$\mathrm{CLB}(\mathrm{L})=0$

$\operatorname{RAB}(\mathbf{L})=0$

NEXT

CLS

GOSUB LOOPY

PRINT

PRINT " In this next part of the study, you will attempt to complete another" PRINT "set of ten anagrams. Everything will be identical to the first part of "

PRINT "study, except that you will select a completely new set of anagrams."

PRINT

PRINT

PRINT " If you would need to read over the instructions again, press $<G>$." PRINT "If anything is unclear to you, please ask the experimenter now. " PRINT "Otherwise, press $<\mathrm{C}>$ to continue."

350 VB\$ = INKEY\$

$\mathrm{HR}=1$

WHILE (VBS $=$ "'"): VBS = INKEY\$: WEND

IF (ASC(VBS) $=103$ ) THEN GOTO START

IF (ASC(VBS) <> 99) THEN GOTO 350

GOSUB SCRDEL:

355 CLS

PRINT : PRINT : PRINT : PRINT : PRINT

COLOR 7, 4 PRINT " Once again, you have the opportunity to select some of the

PRINT" parameters of the task. You will now decide 1) how long you want to

PRINT " 'rest' between each anagram, 2) the subject area from which the

PRINT" anagrams are taken, and 3) the difficulty level of the anagrams. "

COLOR 7,9

GOSUB LOOPY

GOSUB PAUSEC 
370 CLS

PRINT

PRINT "1. How many seconds between each anagram?"

PRINT " (choose any number between $<1>$ and $<9>$ seconds, then press <ENTER >)"

LOCATE 5,5

INPUT ${ }^{m !}, T$

IF $(T<1)$ OR $(T>9)$ THEN GOTO 370

PRINT

380 LOCATE 7, 1

PRINT "2. What topic do you prefer?"

PRINT " press $<1>$ for 'science and nature""

PRINT " press $<2>$ for 'people and events"

LOCATE 11, 5

INPUT '"', STB

IF (STB $<>1$ ) AND (STB $<>2$ ) THEN GOTO 380

PRINT

390 LOCATE 13, 1

PRINT "3. What difficulty level would you like?"

PRINT " press $<1>$ for 'easy"'

PRINT " press $<2>$ for 'challenging' ( 10 bonus points for each anagram solved)"

LOCATE 18, 5

INPUT '"', HH

IF $(\mathrm{HH}<>1)$ AND (HH $<>2)$ THEN GOTO 390

GOSUB LOOPY

GOSUB PAUSEC

CLS

PRINT

PRINT " The anagram task will now begin. There will be no practice trial, so" PRINT "if you are unsure about anything, please ask the experimenter now.

PRINT "Otherwise, press <ENTER> to begin."

GOSUB LOOPY

DO UN'IL INKEYS = CHRS(13)

LOOP

IF ST $=1$ THEN GOTO 490

FOR $1=1$ TO 10

READ S(I).ANA, S(I).CLUE, S(I).SOLUT

NEXT 
490 FOR $1=1$ TO 10

READ S(I).ANA, S(I).CLUE, S(I).SOLUT NEXT

IF STB $=2$ THEN GOTO 500

FOR $1=1$ TO 10

READ S(I).ANA, S(I).CLUE, S(I).SOLUT NEXT

' Main Loop, Second Series

500 FOR L $=1$ TO 10

GOSUB SCRDEL:

CLS

$C L B(L)=0 \quad$ 'initial setting of clue indicator

SKB(L) $=0 \quad$ initial setting of skip indicator

LOCATE 10, 37: COLOR 7, 0

PRINT S(L).ANA

LOCATE 9, 31: PRINT "

LOCATE 11, 31: PRINT "

LOCATE 10, 31: PRINT " "

LOCATE 10, 42: PRINT " "

LOCATE 21, 1: COLOR 7, 4 ' white on red

PRINT " seconds

LOCATE 20, 1

PRINT "

LOCATE 22, 1

PRINT " remaining: "

LOCATE 23, 1

PRINT "

LOCATE 15, 55: COLOR 0, 3

PRINT " Keys to press:

LOCATE 16, 55

PRINT *

LOCATE 17, 55

PRINT " <R> to enter solution "

LOCATE 18, 55

PRINT "

LOCATE 19, 55

PRINT " $<C>$ to get clue

LOCATE 20, 55

PRINT " (costs $30 \mathrm{pts)}$ 
LOCATE 21, 55

PRINT "

LOCATE 22, 55

PRINT " $<S>$ to skip to next "

LOCATE 23, 55

PRINT " anagram

LOCATE 1, 1: COLOR 7,0

PRINT "

LOCATE 2, 1

PRINT " Trial \#"

LOCATE 2, 10: PRINT USING "\#\#"; L

LOCATE 2, 12: PRINT " "

LOCATE 3, 1

PRINT "

' Main timer loop.

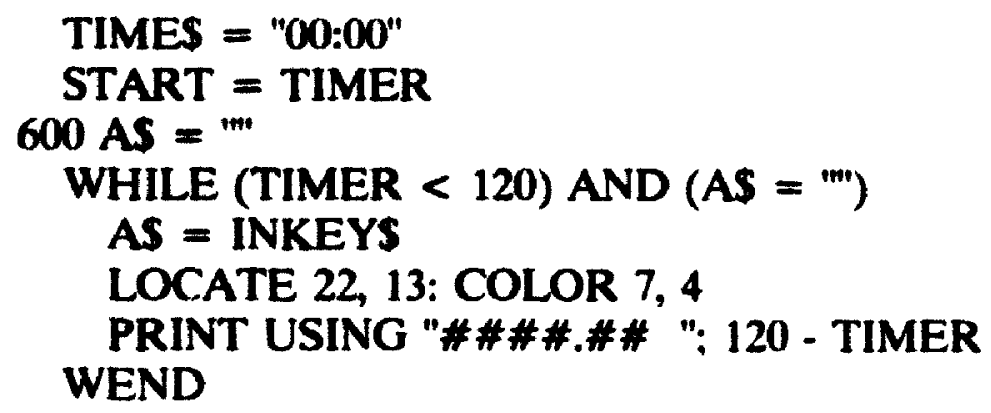

IF (TIMER > 119) THEN GOTO 650 'Time is out, goes to next trial.

IF (ASC(AS) = 99) THEN GOSUB CLUE 'Subroutine for clue (c).

iF $(A S C(A S)=115)$ THEN SKB(L) $=1$ ' skip indicator

IF $(\operatorname{ASC}(A S)=115)$ THEN GOTO 650 ' Subject skips to next trial (s).

LOCATE 15, 35: COLOR 7, 1: PRINT "

IF (ASC(AS) $<>114$ ) GOTO 600

LOCATE 15, 37: COLOR 7, 1

INPUT "', DS

IF $D S=S(L) . S O L U T$ THEN GOSUB CORRECT ELSE GOSUB WRONG

IF DS = S(L).SOLUT THEN GOTO 650 ELSE GOTO 600

650 LOCATE 17, 31: COLOR 7, 0: PRINT " Solution:

LOCATE 18, 37:

'Prints solution at end of trial.

PRINT S(L).SOLUT

LOCATE 18, 31: PRINT "

LOCATE 18, 42: PRINT " 
LOCATE 17, 29: COLOR 7, 9: PRINT " "

LOCATE 17, 4\%: PKINT "

660 LOCATE 21, 26: COLOR 14, 0

PRINT " Score for this trial: "

$\operatorname{SCB}(L)=\operatorname{INT}(119-$ TIMER $)$

IF CI $B(L)=1$ THEN SCB $(L)=\operatorname{SCB}(L)-30$

IF SKB $(L)=1$ THEN SCB(L) $=\operatorname{INT}(0-$ TIMER $)$

IF CLB $(L)=1$ AND SKB(L) $=1$ THEN SCB $(L)=($ INT $(0-$ TIMER $))-30$

LOCATE 21, 49: PRINT SCB(L)

TIMES = "00:00"

START $=$ TIMER

WHILE (TIMER < 5)

WEND

- Program pauses for 5 secs.

IF $L=10$ GOTO 700

COLOR 7,9

CLS

LOCATE 12, 33: PRINT "Get Ready ... "

TIMES = "00:00"

START = TIMER

WHILE (TIMER < T)

WEND

- Program pauses for $\mathrm{T}$ secs.

700 GOSUB LOOPY

NEXT

$\mathrm{TOTB}=\mathrm{SCB}(1)+\mathrm{SCB}(2)+\mathrm{SCB}(3)+\mathrm{SCB}(4)+\mathrm{SCB}(5)+\mathrm{SCB}(6)+\mathrm{SCB}(7)$

$+\mathrm{SCB}(8)+\mathrm{SCB}(9)+\mathrm{SCB}(10)$

$\mathrm{SKB}=\mathrm{SKB}(1)+\mathrm{SKB}(2)+\mathrm{SKB}(3)+\mathrm{SKB}(4)+\mathrm{SKB}(5)+\mathrm{SKB}(6)+\mathrm{SKB}(7)$

$+\operatorname{SKB}(8)+\operatorname{SKB}(9)+\operatorname{SKB}(10)$

$\mathrm{CLB}=\mathrm{CLB}(1)+\mathrm{CLB}(2)+\mathrm{CLB}(3)+\mathrm{CLB}(4)+\mathrm{CLB}(5)+\mathrm{CLB}(6)+\mathrm{CLB}(7)$

$+\mathrm{CLB}(8)+\mathrm{CLB}(9)+\mathrm{CLB}(10)$

$\mathrm{RAB}=\mathrm{RAB}(1)+\mathrm{RAB}(2)+\mathrm{RAB}(3)+\mathrm{RAB}(4)+\mathrm{RAB}(5)+\mathrm{RAB}(6)+$

$\mathbf{R A B}(7)+\mathbf{R A B}(8)+\mathbf{R A B}(9)+\mathbf{R A B}(10)$

CLS

COLOR 7,1

PRINT

PRINT " This first task is now completed. Press <FNTER > to find out your" PRINT "total score."

GOSUB LOOPY

DO UNTIL INKEYS = CHRS(13) 


\section{LOOP}

GOSUB SCRDEL:

CLS

COLOR 7, 4

CLS

PRINT

PRINT " Participant: \#42"

PRINT

PRINT " Number of Correct"

PRINT " $\quad$ Solutions: $\quad$ "; RAB; "'

PRINT " Number of skips: $\quad$ "; SKB; "'

PRINT " Number of clues: $\quad$ "; CLB; "'m

PRINT

PRINT " Total Score: $\quad$ "; TOTB; "'

GOSUB LOOPY

GOSUB PAUSEC

COLOR 7, 9

CLS

PRINT

PRINT " Please tell your experimenter that you are ready for the next task."

730 CLS

INPUT " : ", PWS

IF PWS $<>$ "end" THEN GOTO 730

'Copy data to disk

OPEN "C:ANAGRAM.DAT" FOR APPEND AS \#1

PRINT \# 1, SN; RA; TOT; CL; SK; RAB; TOTB; CLB; SKB

CLOSE \#1

LOCATE 23, 1: INPUT "', Z

END

'Subroutines.

LOOPY:

LP1: IF INKEY\$ $<>$ '"' THEN GOTO LP1:

RETURN

PAUSEC:

LOCATE 22, 27: PRINT "Press <ENTER> to continue" 
DO UNTIL INKEY\$ = CHR\$(13)

LOOP

RETURN

SCRDEL:

CLS

TIMES = "00:00"

START = TIMER

WHILE (TIMER $<.5$ )

WEND

RETURN

CLUE:

' Gives clue (middle letter)

LOCATE 13, 37: COLOR 7, 0

PRINT "

LOCATE 13, 19

PRINT " Middle Letter: "

LOCATE 13, 39: COLOR 7, 0

PRINT S(L).CLUE

$C L(L)=1: C L B(L)=1 \quad$ 'notes to computer that clue was triggered

RETURN

CORRECT:

LOCATE 17, 28: COLOR 7, 9

PRINT "

LOCATE 17, 30: COLOR 7, 0: PRINT " You are correct! "

BEEP

$\operatorname{RA}(L)=1: \operatorname{RAB}(L)=1 \quad$ 'notes to computer right answer given

RETURN

WRONG:

LOCATE 17, 29: COLOR 7, 0: PRINT "Incorrect. Try again." RETURN

DATA gbtio, g, bigot, owamn, m, woman, ordcw, o, crowd

DATA fteih, i, thief, gifth, $g$, fight, ftlri, i, flirt

DATA fiyra, $i$, fairy, criav, $c$, vicar, cnhlu, $n$, lunch

DATA trhim, r, mirth

DATA iogic, g, logic, githl, $g$, light, srivu, r, virus

DATA hnduo, u, hound, itruf, u, fruit, iptlu, l, tulip

DATA ofrec, $r$, force, noehy, $n$, honey, retiv, $v$, rivet

DATA yaorv, a, ovary

DATA ownlc, o, clow m, augrd, a, guard, eundc, n, dunce

DATA zltwa, 1, waltz, ouste, o, scout, hslac, a, clash 
DATA ongya, o, agony, kasnc, a, snack, emycr, r, mercy DATA bnhuc, $n$, bunch

DATA botir, b, orbit, hsafl, a, flash, htiwd, d, width DATA ijnot, $i$, joint, haeny, e, hyena, phted, p, depth DATA hecpr, r, perch, tcuon, u, count, plimb, i, blimp DATA chitp, $t$, pitch 


\section{APPENDIX F}

Analysis of Variance Tables - Experiment 1 


\section{Analysis of Variance Summary Table}

Experiment 1

Direction-DV as a function of direction, structure, and self-esteem

\begin{tabular}{lrrrr}
\hline Source & SS & df & $F$ & $p$ \\
\hline Direction (A) & 410.66 & 1 & 33.30 & .000 \\
Structure (B) & .70 & 1 & .17 & .682 \\
SE (C) & .13 & 1 & .03 & .861 \\
A x B & 15.68 & 1 & 3.81 & .056 \\
A x C & .03 & 1 & .01 & .937 \\
B x C & 2.89 & 1 & .70 & .406 \\
A x B X C & 13.68 & 1 & 3.33 & .406 \\
Error & 209.77 & 51 & & \\
\hline
\end{tabular}


Analysis of Variance Summary Table

Experiment 1

Structure-DV as a function of direction, structure, and self-esteem

\begin{tabular}{lrrrr}
\hline Source & SS & df & $F$ & $p$ \\
\hline Direction (A) & 5.67 & 1 & 1.56 & .216 \\
Structure (B) & 116.15 & 1 & 31.99 & .000 \\
SE (C) & 16.37 & 1 & 4.51 & .039 \\
A x B & 1.94 & 1 & 3.81 & .056 \\
A x C & .23 & 1 & .01 & .937 \\
B x C & .63 & 1 & .70 & .406 \\
A x B X & 4.21 & 1 & 1.16 & .679 \\
Error & 185.15 & 51 & & \\
\hline
\end{tabular}




\section{Analysis of Variance Summary Table}

Experiment 1

Mood Scale as a function of direction, structure, and self-esteem

\begin{tabular}{lrrrr}
\hline Source & SS & df & $F$ & $p$ \\
\hline Direction (A) & 2.51 & 1 & .91 & .338 \\
Structure (B) & 7.44 & 1 & 2.68 & .110 \\
SE (C) & .44 & 1 & .16 & .694 \\
A x B & .22 & 1 & .08 & .782 \\
A x C & .79 & 1 & .28 & .598 \\
B x C & .76 & 1 & .27 & .605 \\
A x B x C & 1.69 & 1 & .61 & .439 \\
Error & 102.60 & 37 & & \\
\hline
\end{tabular}




\section{Analysis of Variance Summary Table}

Experiment 1

Disappointment-relieved rating as a funct ${ }^{2}$ n of direction, structure, and self-esteem

\begin{tabular}{lcccc} 
Source & SS & df & $\boldsymbol{F}$ & $\boldsymbol{p}$ \\
\hline Direction (A) & 27.82 & 1 & 4.63 & .038 \\
Structure (B) & 8.57 & 1 & 1.42 & .240 \\
SE (C) & 5.69 & 1 & .95 & .337 \\
A x B & .21 & 1 & .04 & .852 \\
A x C & .13 & 1 & .02 & .884 \\
B x C & .04 & 1 & .01 & .933 \\
A x B x C & 9.78 & 1 & 1.63 & .210 \\
Error & 222.50 & 37 & & \\
\hline
\end{tabular}


Analysis of Variance Summary Table

Experiment 1

Attribution rating (own responsibility) as a function of direction, structure, and self-esteem

\begin{tabular}{lcccc}
\hline Source & SS & df & $F$ & $p$ \\
\hline Direction (A) & .37 & 1 & .05 & .827 \\
Structure (B) & .33 & 1 & .04 & .837 \\
SE (C) & 4.55 & 1 & .60 & .443 \\
A x B & .31 & 1 & .04 & .840 \\
A X C & 10.17 & 1 & 1.35 & .254 \\
B x C & 21.25 & 1 & 2.81 & .103 \\
A x B x C & 6.34 & 1 & .84 & .366 \\
Error & 264.58 & 35 & & \\
\hline
\end{tabular}


Analysis of Variance Summary Table

Experiment 1

Attribution rating (others' responsibility) as a function of

direction, structure, and self-esteem

\begin{tabular}{lcccc}
\hline Source & SS & df & $F$ & $p$ \\
\hline Direction (A) & .42 & 1 & .05 & .820 \\
Structure (B) & .16 & 1 & .02 & .887 \\
SE (C) & 3.13 & 1 & .39 & .534 \\
A x B & 2.44 & 1 & .31 & .583 \\
A x C & 21.42 & 1 & 2.70 & .110 \\
B x C & 21.49 & 1 & 2.71 & .109 \\
A x B x C & .26 & 1 & .03 & .857 \\
Error & 278.10 & 35 & & \\
\hline
\end{tabular}




\section{Analusis of Variance Summary Table}

Experiment 1

Attribution rating (controllability) as a function of direction, structure, and self-esteem

\begin{tabular}{lcccc}
\hline Source & SS & df & $F$ & $p$ \\
\hline Direction (A) & 13.03 & 1 & 1.50 & .228 \\
Structure (B) & 25.57 & 1 & 2.95 & .095 \\
SE (C) & 6.91 & 1 & .80 & .378 \\
A X B & .02 & 1 & .00 & .987 \\
A x C & 2.75 & 1 & .32 & .577 \\
B x C & 2.92 & 1 & .34 & .565 \\
A x B x C & .68 & 1 & .08 & .781 \\
Error & 303.18 & 35 & & \\
\hline
\end{tabular}


Analysis of Variance Summary Table

Experiment 1

Attribution rating (avoidability) as a function of direction, structure, and self-esteem

\begin{tabular}{lcccc} 
Source & SS & df & $F$ & $p$ \\
\hline Direction (A) & 22.37 & 1 & 2.22 & .145 \\
Structure (B) & .35 & 1 & .04 & .853 \\
SE (C) & 37.37 & 1 & 3.70 & .062 \\
A x B & 9.64 & 1 & .96 & .335 \\
A x C & 1.18 & 1 & .12 & .734 \\
B x C & .32 & 1 & .03 & .860 \\
A x B X C & 8.60 & 1 & .85 & .362 \\
Error & 353.10 & 35 & & \\
\hline
\end{tabular}




\section{APPENDIX G}

Anabysis of Variance Tables - Experiment 2 


\section{Analysis of Variance Summary Table}

Experiment 2

Direction-DV as a function of direction, structure, and self-esteem

\begin{tabular}{lrrrr} 
Source & SS & df & $F$ & $p$ \\
\hline Direction (A) & 808.07 & 1 & 295.38 & .000 \\
Structure (B) & .86 & 1 & .31 & .578 \\
SE (C) & 3.27 & 1 & 1.20 & .278 \\
A x B & 1.06 & 1 & .39 & .536 \\
A x C & 13.88 & 1 & 5.07 & .028 \\
B X C & 1.43 & 1 & .52 & .473 \\
A x B x C & 1.05 & 1 & .38 & .538 \\
Error & 169.62 & 62 & & \\
\hline
\end{tabular}


Analysis of Variance Summary Table

Experiment 2

Structure-DV as a function of direction, structure, and self-esteem

\begin{tabular}{lrrrr}
\hline Source & SS & df & $F$ & $p$ \\
\hline Direction (A) & 2.46 & 1 & 1.01 & .318 \\
Struciure (B) & 617.55 & 1 & 254.51 & .000 \\
SE (C) & .08 & 1 & .03 & .857 \\
A x B & 6.93 & 1 & 2.86 & .096 \\
A x C & .26 & 1 & .11 & .743 \\
B x C & 8.06 & 1 & 3.32 & .073 \\
A x B x C & 3.29 & 1 & 1.36 & .248 \\
Error & 150.44 & 62 & & \\
\hline
\end{tabular}


Analysis of Variance Summary Table

Experiment 2

Mood Scale as a function of direction, structure, and self-esteem

\begin{tabular}{lcccc}
\hline Source & SS & df & $F$ & $p$ \\
\hline Direction (A) & 6.81 & 1 & 5.47 & .023 \\
Structure (B) & 2.00 & 1 & 1.61 & .209 \\
SE (C) & .03 & 1 & .02 & .881 \\
A x B & .65 & 1 & .52 & .474 \\
A x C & .04 & 1 & .03 & .855 \\
B x C & 5.26 & 1 & 4.23 & .044 \\
A x B x C & .32 & 1 & .26 & .613 \\
Error & 77.25 & 62 & & \\
\hline
\end{tabular}


Analysis of Variance Summary Table

Experiment 2

Disappointment-relieved rating as a function of direction, structure, and self-esteem

\begin{tabular}{lcccc} 
Source & SS & df & $F$ & $p$ \\
\hline Direction (A) & 18.27 & 1 & 5.02 & .029 \\
Structure (B) & 9.34 & 1 & 2.57 & .114 \\
SE (C) & 1.11 & 1 & .31 & .582 \\
A x B & .21 & 1 & .06 & .810 \\
A x C & .55 & 1 & .15 & .698 \\
B x C & .22 & 1 & .06 & .808 \\
A x B x C & 1.56 & 1 & .44 & .510 \\
Error & 225.59 & 62 & & \\
\hline
\end{tabular}




\section{Analysis of Variance Summary Table}

Experiment 2

Commissional Intention Index as a function of direction, structure, and self-esteem

\begin{tabular}{lrccc}
\hline Source & SS & df & $F$ & $p$ \\
\hline Direction (A) & 13.21 & 1 & 4.07 & .048 \\
Structure (B) & .15 & 1 & .05 & .832 \\
SE (C) & .60 & 1 & .19 & .668 \\
A x B & 12.34 & 1 & 3.80 & .056 \\
A x C & 4.26 & 1 & 1.31 & .257 \\
B x C & .34 & 1 & .10 & .748 \\
A x B x C & 8.01 & 1 & 2.47 & .121 \\
Error & 201.39 & 62 & & \\
\hline
\end{tabular}




\section{Analysis of Variance Summary Table}

Experiment 2

Omissional Intention Index as a function of direction, structure, and self-esteem

\begin{tabular}{lcccc}
\hline Source & SS & df & $F$ & $p$ \\
\hline Direction (A) & 11.47 & 1 & 3.50 & .066 \\
Structure (B) & .01 & 1 & .00 & .956 \\
SE (C) & 6.14 & 1 & 1.87 & .176 \\
A x B & 1.92 & 1 & .58 & .448 \\
A x C & .06 & 1 & .02 & .897 \\
B x C & 22.27 & 1 & 6.79 & .011 \\
A x B X C & 4.77 & 1 & 1.46 & .232 \\
Error & 201.40 & 62 & & \\
\hline
\end{tabular}


Analysis of Variance Summary Table

Experiment 2

Grade Expectancy as a function of direction, structure, and self-esteem

\begin{tabular}{lcccc}
\hline Source & SS & df & $F$ & $p$ \\
\hline Direction (A) & 332.03 & 1 & 7.49 & .008 \\
Structure (B) & 33.32 & 1 & .75 & .389 \\
SE (C) & 6.67 & 1 & .15 & .700 \\
A x B & 12.97 & 1 & .29 & .591 \\
A x C & .51 & 1 & .01 & .915 \\
B x C & 12.14 & 1 & .27 & .603 \\
A x B x C & 14.16 & 1 & .32 & .574 \\
Error & 2750.31 & 62 & & \\
\hline
\end{tabular}




\section{Analysis of Variance Summary Table}

Experiment 2

Attribution rating (own responsibility) as a function of direction, structure, and self-esteem

\begin{tabular}{lllll}
\hline Source & SS & df & $F$ & $p$ \\
\hline Direction (A) & 27.05 & 1 & 8.81 & .004 \\
Structure (B) & 4.43 & 1 & 1.44 & .234 \\
SE (C) & 2.02 & 1 & .66 & .420 \\
A x B & 2.41 & 1 & .78 & .379 \\
A x C & 2.15 & 1 & .70 & .405 \\
B x C & 9.52 & 1 & 3.10 & .083 \\
A x B x C & .19 & 1 & .06 & .805 \\
Error & 190.35 & 62 & & \\
\hline
\end{tabular}




\section{Analysis of Variance Summary Table}

Experiment 2

Attribution rating (others' responsibility) as a function of direction, structure, and self-esteem

\begin{tabular}{|c|c|c|c|c|}
\hline Source & SS & df & & $p$ \\
\hline Direction (A) & 1.34 & & .23 & .636 \\
\hline Structure (B) & 3.69 & 1 & .62 & .443 \\
\hline SE (C) & & 1 & .73 & .397 \\
\hline$A \times B$ & 8.81 & 1 & 1.49 & .227 \\
\hline$A \times C$ & 1.05 & 1 & .18 & .675 \\
\hline$B \times C$ & 1.08 & 1 & .18 & .671 \\
\hline$A \times B \times C$ & .09 & 1 & .02 & .902 \\
\hline Error & 367.42 & 62 & & \\
\hline
\end{tabular}




\section{Analysis of Variance Summary Table}

Experiment 2

Attribution rating (controllability) as a function of direction, structure, and self-esteem

\begin{tabular}{lcccc}
\hline Source & SS & df & $F$ & $p$ \\
\hline Direction (A) & 1.68 & 1 & .38 & .541 \\
Structure (B) & .34 & 1 & .08 & .782 \\
SE (C) & 9.22 & 1 & 2.09 & .154 \\
A x B & .73 & 1 & .17 & .686 \\
A x C & 1.15 & 1 & .26 & .612 \\
B x C & .68 & 1 & .15 & .697 \\
A x B X C & 3.74 & 1 & .85 & .361 \\
Error & 274.21 & 62 & & \\
\hline
\end{tabular}


Analysis of Variance Summary Table

Experiment 2

Attribution rating (avoidability) as a function of direction, structure, and self-esteem

\begin{tabular}{lcccc}
\hline Source & SS & df & $F$ & $p$ \\
\hline Direction (A) & 21.43 & 1 & 4.48 & .038 \\
Structure (B) & .22 & 1 & .05 & .830 \\
SE (C) & 20.40 & 1 & 4.27 & .043 \\
A x B & 9.35 & 1 & 1.96 & .167 \\
A x C & 2.29 & 1 & .48 & .492 \\
B x C & 3.75 & 1 & .78 & .379 \\
A x B $~$ & 8.48 & 1 & 1.77 & .188 \\
Error & 296.51 & 62 & & \\
\hline
\end{tabular}




\section{APPENDIX H \\ Analysis of Variance Tables - Experiment 3}


Analysis of Variance Summan Table

Experiment 3

Direction-DV as a function of direction, structure, and self-esteem

\begin{tabular}{lcccc}
\hline Source & SS & df & $F$ & $p$ \\
\hline Direction (A) & 454.41 & 1 & 225.09 & .000 \\
Structure (B) & .15 & 1 & .08 & .783 \\
SE (C) & .51 & 1 & .26 & .613 \\
A x B & 6.10 & 1 & 3.11 & .084 \\
A x C & .15 & 1 & .08 & .783 \\
B x C & 2.62 & 1 & 1.34 & .253 \\
A x B x C & 1.63 & 1 & .83 & .366 \\
Error & 102.02 & 52 & & \\
\hline
\end{tabular}




\section{Analysis of Variance Summary Table}

Experiment 3

Structure-DV as a function of direction, structure, and self-esteem

\begin{tabular}{lcccc}
\hline Source & SS & df & $F$ & $p$ \\
\hline Direction (A) & .16 & 1 & .07 & .800 \\
Structure (B) & 380.02 & 1 & 153.63 & .000 \\
SE (C) & .02 & 1 & .01 & .939 \\
A x B & .00 & 1 & .00 & .986 \\
A x C & 1.61 & 1 & .65 & .424 \\
B x C & 1.36 & 1 & .55 & .462 \\
A x B X C & .39 & 1 & .16 & .694 \\
Error & 128.63 & 52 & & \\
\hline
\end{tabular}




\section{Analysis of Variance Summary Table}

Experiment 3

Mood Scale as a function of direction, structure, and self-esteem

\begin{tabular}{lcccc}
\hline Source & SS & df & $F$ & $p$ \\
\hline Direction (A) & .00 & 1 & .00 & .984 \\
Structure (B) & .00 & 1 & .00 & .999 \\
SE (C) & .96 & 1 & .78 & .382 \\
A X B & .03 & 1 & .03 & .873 \\
A x C & 1.14 & 1 & .92 & .342 \\
B x C & 2.96 & 1 & 2.38 & .129 \\
A x B x C & 6.27 & 1 & 5.05 & .029 \\
Error & 64.53 & 52 & & \\
\hline
\end{tabular}




\section{Analysis of Variance Summary Table}

Experiment 3

Disappointment-relieved rating as a function of direction, structure, and self-esteem

\begin{tabular}{|c|c|c|c|c|}
\hline Source & SS & df & $\boldsymbol{F}$ & $p$ \\
\hline Direction (A) & .29 & 1 & .16 & .691 \\
\hline Structure (B) & .82 & 1 & .46 & .502 \\
\hline $\mathrm{SE}(\mathrm{C})$ & 1.28 & 1 & .72 & .402 \\
\hline$A \times B$ & 2.29 & 1 & 1.28 & .264 \\
\hline$A \times C$ & .08 & 1 & .04 & .838 \\
\hline $\mathbf{B} \times \mathbf{C}$ & .61 & 1 & .34 & .561 \\
\hline$A \times B \times C$ & 4.98 & 1 & 2.78 & .101 \\
\hline Error & 93.05 & 52 & & \\
\hline
\end{tabular}




\section{Analysis of Variance Summary Table}

Experiment 3

Expectancy Rating as a function of direction, structure, and self-esteem

\begin{tabular}{lcccc}
\hline Source & SS & df & $F$ & $p$ \\
\hline Direction (A) & 1.12 & 1 & .26 & .612 \\
Structure (B) & .83 & 1 & 1.83 & .183 \\
SE (C) & .16 & 1 & .34 & .562 \\
A x B & .19 & 1 & .42 & .520 \\
A x C & .23 & 1 & .51 & .481 \\
B x C & .05 & 1 & .11 & .747 \\
A x B x C & 1.73 & 1 & .067 & .067 \\
Error & 23.28 & 51 & & \\
\hline
\end{tabular}




\section{Analysis of Variance Summary Table}

Experiment 3

Improvement in total score as a function of direction, structure, and self-esteem

\begin{tabular}{lllll}
\hline Source & SS & df & $F$ & $p$ \\
\hline Direction (A) & 267.51 & 1 & 4.72 & .034 \\
Structure (B) & 716.79 & 1 & 12.65 & .001 \\
SE (C) & 364.52 & 1 & 6.43 & .014 \\
A $\times$ B & 162.37 & 1 & 2.87 & .097 \\
A x C & 11.13 & 1 & .02 & .889 \\
B x C & 103.19 & 1 & 1.82 & .183 \\
A & 324.32 & 1 & 5.72 & .020 \\
Error & 2947.21 & 52 & & \\
\hline
\end{tabular}

Note. Sum of square values are $\mathrm{x} 1000$. 


\section{Analysis of Variance Summary Table}

Experiment 3

Attribution rating (own responsibility) as a function of direction, structure, and self-esteem

\begin{tabular}{lcccc}
\hline Source & SS & df & $F$ & $p$ \\
\hline Direction (A) & 2.11 & 1 & .49 & .489 \\
Structure (B) & .42 & 1 & .10 & .758 \\
SE (C) & 5.04 & 1 & 1.16 & .286 \\
A $\times$ B & 3.19 & 1 & .73 & .395 \\
A x C & .00 & 1 & .00 & .996 \\
B x C & .85 & 1 & .20 & .660 \\
A & .76 & 1 & .17 & .678 \\
Errur & 226.02 & 52 & & \\
\hline
\end{tabular}




\section{Analysis of Variance Summary Table}

\section{Experiment 3}

Attribution rating (task difficulty) as a function of direction, structure, and self-esteem

\begin{tabular}{lcccc}
\hline Source & SS & df & $F$ & $p$ \\
\hline Direction (A) & .25 & 1 & .08 & .779 \\
Structure (B) & 1.35 & 1 & .42 & .518 \\
SE (C) & 1.06 & 1 & .33 & .568 \\
A x B & 11.25 & 1 & 3.53 & .066 \\
A x C & .08 & 1 & .02 & .877 \\
B x C & 47.76 & 1 & 14.99 & .000 \\
A x B x C & 9.80 & 1 & 3.08 & .085 \\
Error & 165.71 & 52 & & \\
\hline
\end{tabular}




\section{Analysis of Variance Summary Table}

Experiment 3

Attribution rating (controllability) as a function of direction, structure, and self-esteem

\begin{tabular}{lcccc}
\hline Source & SS & df & $F$ & $p$ \\
\hline Direction (A) & .42 & 1 & .09 & .764 \\
Structure (B) & .82 & 1 & .18 & .673 \\
SE (C) & 3.54 & 1 & .77 & .382 \\
A x B & 4.49 & 1 & .99 & .325 \\
A x C & 12.86 & 1 & 2.83 & .099 \\
B x C & 2.24 & 1 & .49 & .486 \\
A x B x C & 2.12 & 1 & .47 & .498 \\
Error & 236.58 & 52 & & \\
\hline
\end{tabular}




\section{Analysis of Variance Summary Table}

\section{Experiment 3}

Attribution rating (avoidability) as a function of direction, structure, and self-esteem

\begin{tabular}{lcccc}
\hline Source & SS & df & $F$ & $p$ \\
\hline Direction (A) & 5.67 & 1 & 1.74 & .193 \\
Structure (B) & .15 & 1 & .05 & .831 \\
SE (C) & 2.40 & 1 & .74 & .395 \\
A x B & .01 & 1 & .00 & .967 \\
A x C & .22 & 1 & .07 & .799 \\
B x C & .16 & 1 & .05 & .826 \\
A x B X C & .0. & 1 & .00 & .965 \\
Error & 169.89 & 52 & & \\
\hline
\end{tabular}


APPENDIX I

Raw Data 


\section{Data - Experiment 1}

Column Variable

1.3 Subject number

4 Sex

5 Direction ( 1 =upward, $2=$ downward)

6 Structure ( 1 =additive, $2=$ subtractive)

8 Upward/additive counterfactuals

9 Upward/subtractive counterfactuals

10 Downward/additive counterfactuals

11 Downward/subtractive counterfactuals

13-18 Mood ratings

20-35 Self-esteem ratings

37-46 Self-focus ratings

48 Mention of death in counterfactuals $(0=$ no death, $1=$ death $)$

49 Nonparametric manipulation check $(0=$ correct. $1=$ incorrect $)$

51-54 Attribution ratings 
011111300022525107870460524137181213502043009199 022222000175355226575551774225644067424132005618 03121202005131510768065084008828616741815500 041111910011414116772780561055553216404063106769 051221002044545454442653642372232426226244009599 061122000654456725552442644354665246515156007743 072122000347945446542225113274344216815263007486 081111300089588543584353843365767335505266001622 09121203001121113532573554855252524222225500

101121002322422156463460840478867007418674015557 111222000122535426751451653157374667201167001116 121111320033545222261442742246446244414124005534 131121002154445736752541362256163335523155007732 141211200011815143226454445255457564416162008189 152121003043555878800560364076181505130316006853 162111500044535327661661652454064067712276101715 171212100044345326662631733346265224112226018357 181122001011115107880875844548535500524473011111 19121200023863 it 65660240865046172535604032016859 201221006072827514682452852176867143507252008691 211122000221322234542652751455241244225133002912 221221002052552617551252551267062647433512005821 232121003035315263435764344424448146722143101112 241112310034654616422351632154435132314060011924 251111210044644283414644185457203885130808005949 262222000045545517776663843246364356624454011617 271112440056939365555432552224764232316152016664 281221001033232242233333333344738001505060001847 291111200067999426781451852288181347208065006682 301212100067676724243131432233652212136144014654 311121002078987924461711545241174717211228005681 322222000133335506771560850888288004806080004691 332211310057931127776761746266275117714163002923 342221001087352536553533751376356626416244002891 351121001065655527662342557334447165431265001178 362212000049685924362443652255247234502271017131 371121003035335106652460712187378264535153006829 381112230053332146572146847577773624624033001916 391122000111323165656532753165857723315136006365 $4022220010 \$ 4545425761551645175376213515444018648$ 412112020033647254662432843265286314416172007236 421212410054645635651665841276676217716084015564 431111320042441237771251851077365117708081002112 442122000344535236562343645234656124715523007769 
451211200044445738761142721288277018808087005568 462111100054657704673321872177276126701163002851 471111200055775826764563772273275447524267001911 481122000233625216672661846476456525111064007731 491212230022315217780664843048274145503654006135 501112001055537611466621773523758713747073107719 511111240022438877687357743386286045606160018691 522211200053235164334125433475645326525262006978 532121002022311126780761841076188216211647003878 542122001023238426562757666574166265716162019193 552122000249444853633370740358178108816082001931 562121000244755254533553453446362346435356019599 572121003066465734657762744367363313315242008289 582112010034546326671552756265455335326253001639 592112030032535223452242662365145213627162008777 


\section{Data - Experiment 2}

$\begin{array}{ll}\text { Column } & \text { Variable } \\ 1-2 & \text { Subject number } \\ 3 & \text { Direction (1=upward, } 2=\text { downward }) \\ 4 & \text { Structure }(1=\text { additive, } 2=\text { subtractive }) \\ 5 & \text { Sex }(1=\text { female, } 2=\text { male }) \\ 7 & \text { Upward/additive counterfactuals } \\ 8 & \text { Upward/subtractive counterfactuals } \\ 9 & \text { Downward/additive counterfactuals } \\ 10 & \text { Downward/subtractive counterfactuals } \\ 11-18 & \text { Mood ratings } \\ 19-22 & \text { Attribution ratings } \\ 23-24 & \text { Grade expectancy } \\ 26,28,30 & \text { Commissional intention ratings } \\ 27.29,31 & \text { Omissional intention ratings } \\ 33-56 & \text { Individual difference scale ratings } \\ 58 & \text { Group }\end{array}$


011113000135544558476782768797848626766673268466287271 021112000113111249199759987997763621765443664427755551 031123000224532438587803667918786941368661438797624671 041113000215434129298656877849949287694861218892295231 051122010223545148297709936991994215888872294889689771 061122010545545568543709499576737532543852364564565351 071113000313777589191657684963838282277882287363382281 081112010324567889168753918998827796227881389583172281 091125000177555159179807999997989885199771141499175171 101114000112123257198807979896677186667864777466677681 111119030243525328698753944691778994294981217671298381 121122010365555339199601959895727319978652895588673281 141122000485977467663708868883778222268763637672588641 151114000445555558287753723282828682278884268716288291 161113000722253257117659277955784778848664568556753571 $17121101014554334916676675788477646632883122737735665^{*} 2$ 181210020115535247277706683881839793147972278673198392 191210050676773458678707969793647764288653567442297672 201210040123556658657728998996826446674761599414894152 231220040314545138678707834873878772666972367678787682 241210030243341459157757959997716441639763697665576862 251220030313856458893879979998879766689753553571599572 271210030335555557287707787777764545738753388367573552 281220030143515137186709339968728652238851268773278282 291220050324555469378757649893729248447884428584287382 3112 ! 0040144445459477708925885342247638652678366565912 322120301533754458199658274784768899888991328586288283 342120400155335447468856915238787832598881248885455283 362110200125452468285758852882868255672875364775268473 372110201152837149197859461712713281219879289862884893 382120201565644542822852449837424325566652436465467843 392120201665347554132752854985674777384575255564589573 402110401898859557736806973994828273486665326562496333 412110501245544457188707858995727171367966347524586253 422120300637377759198702272182818691281778277757655463 442120201224334249198758928883262232699856476789876633 452220005325533441199817171421919882192891128882199194 462220002155597627131756949698293759174871837684389584 472210001445454345643809926995737777998869213666478444 482210002133319258773753128896888695544971199734297184 492210007274523397177751956991858193222689387813297394 502210003454547459176853939982728276499659377364327364 512220001485579789188723622954364966648833357366785734 522220002585356453272806937786232524468728262548542544 
532220002113381478263808919889738126388991796879172294 542210003484535457387856311997687191158885199699238284 552210003235533148664853929992262262699969888182698934 5722100053353694582738057799927378366888332639292788874 582220008534524459199751378968253241468775388735676324 602110300796991779296807967999282118949453399999911273 612110100998884678798708986693718192778877289623177373 632120400267417562389552311175372568342652387688634843 651220030445434549198607937896677552345845377558477882 661210030485646479187704723487221242778965668543486832 672120300185525533832909949874849373447872466665258293 682210004555545457199857648981828291248888272827199194 692210004122522459167758119851958494959994577575199594 711220030115515259119809932295775866642951396695599272 722220003436434468697779977892879672187852123563388684

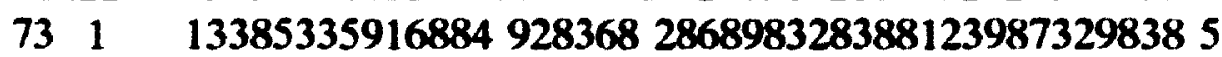

$\begin{array}{lll}74 & 2 & 111552258168809969699919995299871174878128185\end{array}$

$\begin{array}{llll}75 & 2 & 195513459199809911199999979291593113999197195\end{array}$

$\begin{array}{llll}76 & 1 & 111121133333759988897438193327985399313598585\end{array}$

$77 \quad 1 \quad 467334637143703974492324491667921338367374865$

$\begin{array}{llll}78 & 1 & 19251214332360 & 1319198423112914254694664315435\end{array}$

$\begin{array}{lll}79 & 2 & 33554455667765913729 \\ 87688865154862317667298395\end{array}$

$\begin{array}{llll}80 & 2 & 33455352646375 & 7968996637463467786366664499445\end{array}$

$\begin{array}{llll}81 & 2 & 22113433727680899499 & 4826233297973339464847135\end{array}$

$822 \quad 225332259199757949291519771195781198554199195$

$83 \quad 1 \quad 2247233592886228271973629663632422227576646645$

$84 \quad 1 \quad 32454: 354667703232776628688253754667646264485$

$\begin{array}{lll}85 & 2 & 22335535929875 \\ 892869 & 8728718858872297772384485\end{array}$

862120200495755646676754414297796885457651278687395753 871220050134325247278806271883888477297884274664288282 882220102433735369178706133182858897287981128777288394

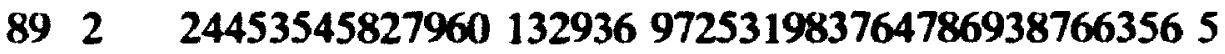
90112300022453545576470883778288875557785778779234285 1 911116000484644337288703939781912394134994279378278481 921116000332113447364708922398186738867842347673647721 $93 \quad 1 \quad 111313127686737657683576781186851249874387475$ $95222000228552825725575222911 \quad 1858888192881228771288594$ 961220020155894549188659939897636442658868387722387342 971210040114342548757736869862886737988772547586377672 982210003513521357476701261752575877298771288785327354 992110301585569567677808969893827233247883248564288863 


\section{Data - Experiment 3}

Column Variable

\section{First Line}

1-3 Subject number

5-6 Anagrams correct, first set

8-11 Total score, first set

13-14 Number of clues used, first set

15-16 Number of skips, first set

19-20 Anagrams correct, second set

22-25 Total score, second set

27-28 Number of clues used, second set

30-31 Number of skips, second set

33

34

35

36

38-45 Mood ratings

46-49 Attribution ratings

50 Expectaricy rating

\section{Second Line}

1-24 Individual difference scale ratings

26 Upward/additive counterfactuals

27 Upward/subtractive counterfactuals

28 Downward/additive counterfactuals

29 Downward/subtractive counterfactuals

30 Mention clue in counterfactuals 
$\begin{array}{lllllllllll}14 & 98 & 3 & 6 & 8 & 461 & 7 & 2 & 1121 & 2753544567663\end{array}$ $675746535487249652128 \times 34100(0)$

$\begin{array}{lllllllllll}2 & 8 & 633 & 0 & 2 & 8 & 805 & 0 & 2 & 1121 & 4556654696984\end{array}$ 87466229765336834247266410000

$\begin{array}{lllllllllll}3 & 6 & 13 ! & 5 & 4 & 8 & 265 & 5 & 1 & 1111 & 3257214443644\end{array}$ 13287583987565545688553730000

$\begin{array}{llllllllllll}4 & 2 & -61 & 0 & 8 & 4 & 84 & 0 & 6 & 1111 & 2255345497384\end{array}$ 37767687588537785865766542000

$\begin{array}{lllllllllll}5 & 4 & 184 & 6 & 6 & 4 & 328 & 1 & 6 & 1121 & 555757557378.3\end{array}$ 396666566888681257673883530001

$\begin{array}{lllllllllll}6 & 1 & -241 & 4 & 9 & 5 & 316 & 3 & 5 & 1121 & 4358773492444\end{array}$ 48291951799812387822822921000

$\begin{array}{lllllllllll}7 & 4 & 374 & 1 & 6 & 8 & 714 & 0 & 2 & 2123 & 1247372596675\end{array}$ 25263912996624783522786900200

$\begin{array}{llllllllllll}8 & 5 & 299 & 2 & 5 & 8 & 636 & 1 & 2 & 2113 & 1747287993935\end{array}$ 37287683848712785971744400200

$\begin{array}{lllllllllll}9 & 3 & 215 & 0 & 7 & 6 & 604 & 0 & 4 & 1222 & 1435212576775\end{array}$ 36776826577743276753973821000

$\begin{array}{lllllllllll}10 & 6 & 530 & 0 & 4 & 8 & 792 & 0 & 2 & 1212 & 3978787893873\end{array}$ 77381776288556783664556603000

$\begin{array}{lllllllllll}12 & 8 & 417 & 6 & 2 & 8 & 561 & 5 & 2 & 1212 & 1685337895764\end{array}$ 72276717788527795422777702000

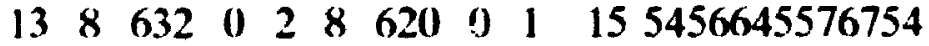
252518564586458956349758

$\begin{array}{lllllllllll}14 & 6 & 388 & 5 & 4 & 9 & 878 & 2 & 1 & 15 & 3454444587764\end{array}$ 746223562897246861877476

$\begin{array}{lllllllllll}15 & 9 & 700 & 3 & 1 & 9 & 559 & 5 & 1 & 15 & 3456645565734\end{array}$ 325324474785976567769743

$\begin{array}{lllllllllll}16 & 8 & 587 & 0 & 2 & 6 & 314 & 0 & 4 & 15 & 7778 \times 388887835\end{array}$ 6625778268844147968344792

$\begin{array}{lllllllllll}18 & 9 & 449 & 8 & 1 & 7 & 293 & 6 & 3 & 1222 & 5957475947664\end{array}$ 891652593984678927869977 (02(K)1

$\begin{array}{lllllllllll}23 & 3 & 1 & 0 & 7 & 7 & 654 & 0 & 3 & 1222 & 2455364598754\end{array}$ 27475713578453876334783703000

$248762 \quad 0 \quad 2 \quad 6 \quad 57000412123959553695472$ 61476752349752691867173502000

$\begin{array}{lllllllllll}25 & 5 & 491 & 0 & 5 & 7 & 713 & 0 & 3 & 1222 & 24484444993885\end{array}$ $77762452887537675655752503060)$

$\begin{array}{llllllllllll}26 & 8 & 747 & 1 & 2 & 6 & 466 & 2 & 4 & 1212 & 3975989992795\end{array}$ $78 \times 8826595847388937849993$ (12000)

$\begin{array}{lllllllllll}27 & 3 & 213 & 0 & 7 & 5 & 471 & 0 & 5 & 1212 & 2951455699875\end{array}$ 284766824887114867338848 (1306)

$\begin{array}{lllllllllll}28 & 7 & 240 & 6 & 1 & 8 & 555 & 3 & 0 & 25 & 4556566566663\end{array}$ 735454356595257562368638 
$\begin{array}{lllllllllll}29 & 4 & 206 & 2 & 6 & 7 & 396 & 3 & 3 & 2214 & 5556655586664\end{array}$ 32253356476535475723522500010

$\begin{array}{lllllllllll}30 & 6 & 552 & 1 & 4 & 4 & 320 & 0 & 6 & 2113 & 3555393586834\end{array}$ 46271457196616684832846400400

$\begin{array}{lllllllllll}32 & 7 & 605 & 0 & 3 & 4 & 94 & 4 & 6 & 2224 & 7857778886824\end{array}$ 78393722278533273183772800040

$\begin{array}{lllllllllll}33 & 9 & 890 & 0 & 1 & 6 & 324 & 3 & 4 & 2214 & 2555455556624\end{array}$ 77131214798633993722737400010

$\begin{array}{lllllllllll}35 & 7 & 672 & 1 & 3 & 7 & 515 & 4 & 3 & 2214 & 2642342532875\end{array}$ 443664567966246066625447300061

$\begin{array}{llllllllllll}36 & 5 & 341 & 3 & 5 & 5 & 384 & 1 & 5 & 1111 & 3798745592454\end{array}$ 17372852687722683542874630000

$\begin{array}{lllllllllll}38 & 2 & -218 & 5 & 8 & 5 & 365 & 1 & 5 & 1121 & 2652215558224\end{array}$ 75135338777256596135347430001

$\begin{array}{lllllllllll}40 & 4 & 67 & 2 & 6 & 4 & 74 & 2 & 6 & 2214 & 2955555594174\end{array}$ 38283812278832898371783920000

$\begin{array}{llllllllllll}42 & 8 & 762 & 3 & 2 & 7 & 735 & 1 & 3 & 2214 & 2555355797354\end{array}$ 38688151669733796721872900021

$\begin{array}{lllllllllll}43 & 5 & 219 & 5 & 5 & 6 & 316 & 5 & 4 & 2123 & 1344354678764\end{array}$ 65271119767697727185229300300

$\begin{array}{lllllllllll}44 & 5 & 238 & 0 & 5 & 8 & 519 & 0 & 2 & 25 & 3348433646443\end{array}$ 3537536666455544457555643

$\begin{array}{lllllllllll}45 & 6 & 386 & 2 & 1 & 10 & 713 & 5 & 0 & 2224 & 3582495573663\end{array}$ 77572612585642785885875700040

$\begin{array}{lllllllllll}46 & 4 & 232 & 0 & 6 & 7 & 516 & 4 & 3 & 1121 & 2652272996874\end{array}$ 68281613668536775633882530001

$\begin{array}{llllllllllll}47 & 6 & 300 & 2 & 4 & 5 & 238 & 3 & 4 & 15 & 3632455577351\end{array}$ 6525547268885583646235465

$\begin{array}{lllllllllll}48 & 7 & 92 & 5 & 3 & 6 & 273 & 4 & 2 & 15 & 2356695598363\end{array}$

183869718898217886229718

$\begin{array}{lllllllllll}49 & 3 & -157 & 3 & 7 & 5 & 484 & 0 & 5 & 2113 & 1111141394364\end{array}$ 77676853777533887874768800201

$\begin{array}{lllllllllll}50 & 4 & 336 & 0 & 6 & 8 & 855 & 0 & 2 & 2123 & 3954435795864\end{array}$ 37364534577634775655676700300

$\begin{array}{llllllllllll}51 & 5 & 87 & 8 & 5 & 7 & 498 & 5 & 3 & 2113 & 5857775748853\end{array}$ 33134966797526656355635800200

$\begin{array}{lllllllllll}52 & 3 & 203 & 1 & 7 & 7 & 503 & 0 & 0 & 15 & 4968454862253\end{array}$ 856372893687577678885554

$\begin{array}{lllllllllll}53 & 5 & 117 & 7 & 5 & 5 & 195 & 5 & 5 & 2123 & 4556565597964\end{array}$ 86647423789512866895786600201

$\begin{array}{lllllllllll}54 & 5 & 412 & 0 & 5 & 7 & 626 & 0 & 3 & 2113 & 3553726786574\end{array}$ 38683772789532493635882800400

$\begin{array}{llllllllllll}55 & 5 & 541 & 0 & 5 & \text { o } & 781 & 2 & 2 & 2113 & 2955455597974\end{array}$ 67681753899742867224986700300 
$\begin{array}{lllllllllll}56 & 2 & -317 & 8 & 8 & 5 & 8 & 9 & 5 & 2224 & 2358333466474\end{array}$ 38777817867725676775573700200

$\begin{array}{lllllllllll}57 & 7 & 140 & 4 & 3 & 7 & 61 & 7 & 3 & 2214 & 2311353552384\end{array}$ 95435456457789391855455500010

$\begin{array}{lllllllllll}58 & 4 & -234 & 5 & 5 & 6 & 155 & 9 & 3 & 15 & 2357735556674\end{array}$ 977575773833532457757255

$\begin{array}{lllllllllll}59 & 1 & -173 & 1 & 9 & 4 & 250 & 0 & 6 & 2123 & 3269872475375\end{array}$ 38197627498533787836545600600

$\begin{array}{lllllllllll}60 & 5 & 297 & 5 & 5 & 5 & 292 & 5 & 5 & 2113 & 3259975773654\end{array}$ 78677358887457843787883200301

$\begin{array}{lllllllllll}61 & 3 & -83 & 2 & 7 & 5 & 443 & 0 & 5 & 2113 & 4568695753354\end{array}$ 53556513999821999658835600301

$\begin{array}{llllllllllll}62 & 3 & -116 & 8 & 7 & 7 & 500 & 3 & 3 & 1121 & 1452525588765\end{array}$ 65655385598527882757479360001

$\begin{array}{llllllllllll}63 & 4 & 276 & 2 & 6 & 8 & 817 & 0 & 2 & 1111 & 2345344577774\end{array}$ 73336178447677746777738340000

$\begin{array}{lllllllllll}64 & 7 & 161 & 8 & 3 & 7 & 319 & 5 & 3 & 1222 & 2455473775553\end{array}$ 37687556478633565632763802001

$\begin{array}{lllllllllll}65 & 7 & 301 & 5 & 3 & 6 & 200 & 5 & 4 & 1212 & 2554443487853\end{array}$ 75456656537686774627664403000

$\begin{array}{lllllllllll}66 & 3 & 17 & 4 & 7 & 5 & 214 & 6 & 5 & 15 & 5643343545444\end{array}$ 424337544465477666467655

$\begin{array}{lllllllllll}67 & 9 & 880 & 2 & 1 & 10 & 860 & 2 & 0 & 1212 & 5558588817113\end{array}$ 35766744667524464644464601000

$\begin{array}{lllllllllll}68 & 5 & -16 & 6 & 2 & 5 & 321 & 3 & 2 & 25 & 8979989982475\end{array}$

192738136986384829195846

$\begin{array}{lllllllllll}69 & 3 & -81 & 5 & 7 & 6 & 467 & 2 & 4 & 1212 & 2342453578674\end{array}$ 78233952878782898231995804001

$\begin{array}{lllllllllll}70 & 3 & 45 & 1 & 7 & 7 & 457 & 4 & 3 & 1222 & 3355342596774\end{array}$ 47664863678837787663474802000

$\begin{array}{lllllllllll}71 & 2 & 21 & 1 & 8 & 6 & 269 & 5 & 4 & 1212 & 1953556678663\end{array}$ 16385822878811885622997502000

$\begin{array}{llllllllllll}72 & 1 & -111 & 1 & 9 & 1 & -57 & 2 & 9 & 2214 & 2254687887733\end{array}$ 54643633975623994547886300010

$\begin{array}{lllllllllll}73 & 7 & 468 & 4 & 3 & 8 & 846 & 0 & 0 & 1111 & 3575736772885\end{array}$ 67661311897826977791773740000

$\begin{array}{lllllllllll}74 & 4 & -13 & 6 & 5 & 6 & 98 & 5 & 4 & 2123 & 4565545434444\end{array}$ 39685822779697795652884900200

$\begin{array}{lllllllllll}75 & 8 & 723 & 2 & 2 & 8 & 836 & 0 & 2 & 1111 & 4455545675674\end{array}$ 27462746484674883664764530001

$\begin{array}{lllllllllll}76 & 4 & 332 & 0 & 6 & 7 & 621 & 3 & 3 & 1111 & 5457515877754\end{array}$ 42141312998513774172999840001

$\begin{array}{lllllllllll}77 & 6 & 463 & 1 & 4 & 9 & 880 & 1 & 1 & 2113 & 4471839987764\end{array}$ 29894831942623786761983800300 
238

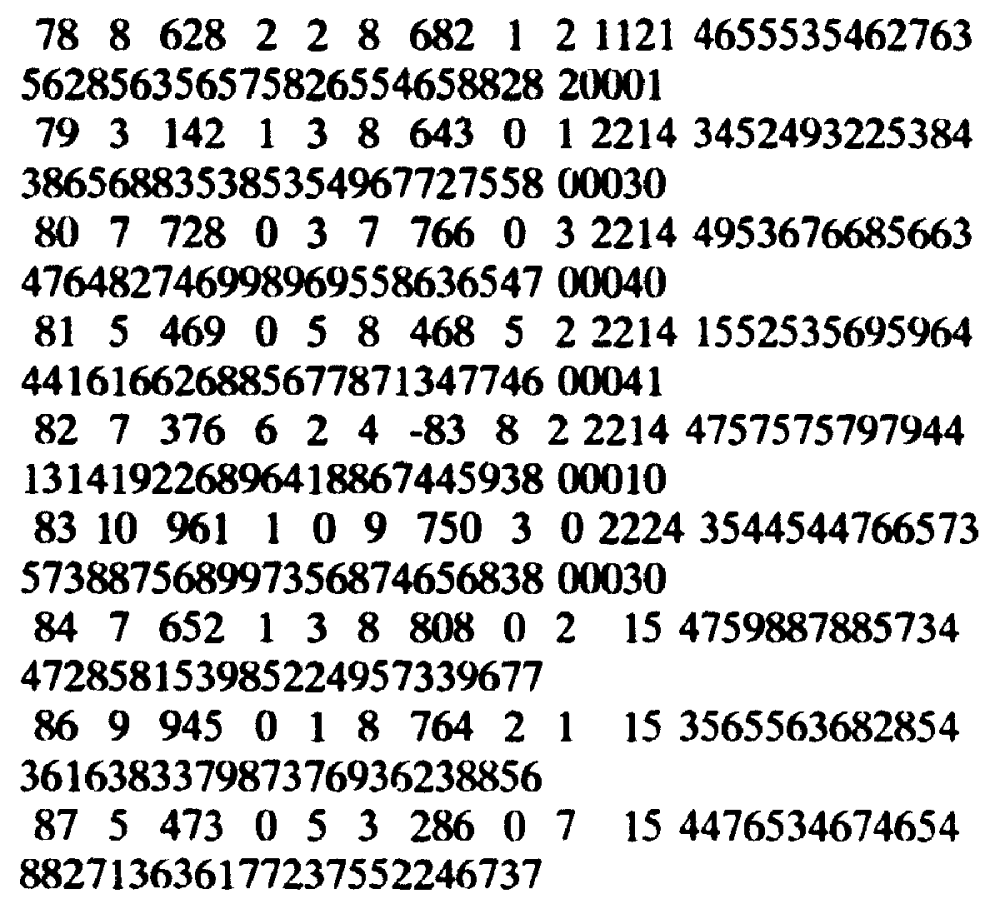

